Western University

Scholarship@Western

Digitized Theses

Digitized Special Collections

1986

\title{
A Tauberian Theorem Concerning Borel-type And Cesaro Methods Of Summability
}

Tom Nik Markovich

Follow this and additional works at: https://ir.lib.uwo.ca/digitizedtheses

\section{Recommended Citation}

Markovich, Tom Nik, "A Tauberian Theorem Concerning Borel-type And Cesaro Methods Of Summability" (1986). Digitized Theses. 1553.

https://ir.lib.uwo.ca/digitizedtheses/1553

This Dissertation is brought to you for free and open access by the Digitized Special Collections at Scholarship@Western. It has been accepted for inclusion in Digitized Theses by an authorized administrator of Scholarship@Western. For more information, please contact tadam@uwo.ca, wlswadmin@uwo.ca. 


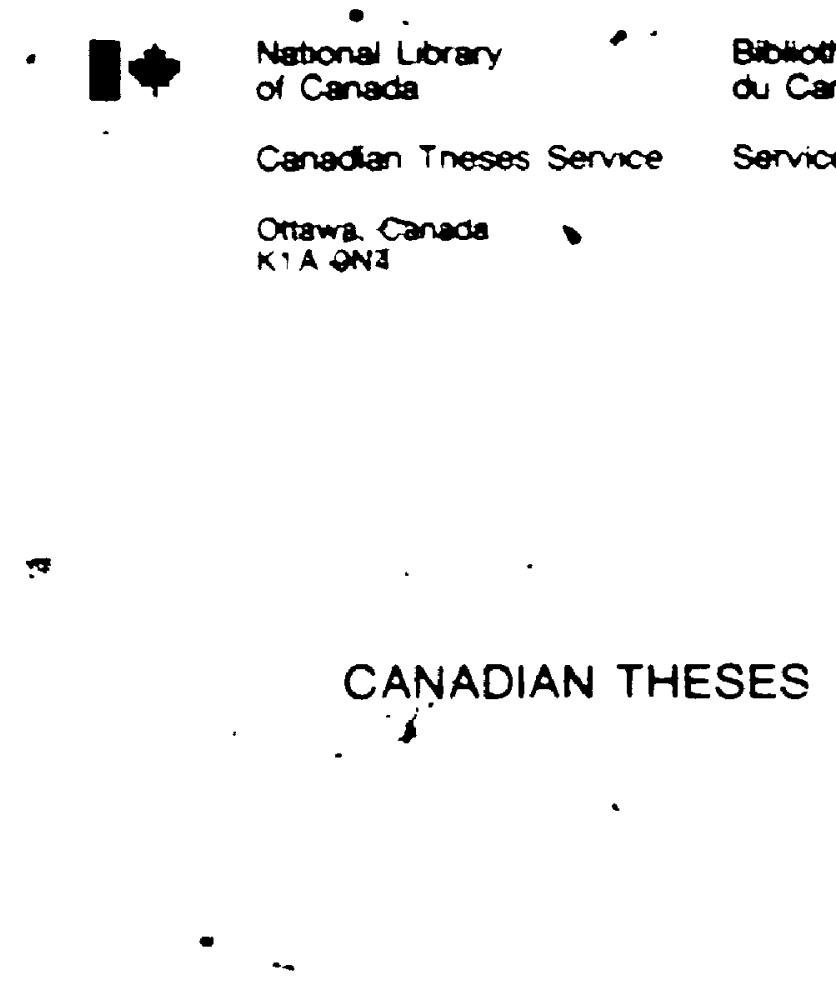

\section{NOTKE -}

The quality of this microfiche is heavily dependent upon the quatity of the original thesis submitted for microfilming Every effor has been made to ensure the highest quality of reproduction possibye

It mages are missing. contact the university which granted the degree

Some pages may have moistinct print especially if the original pages were typed with a poor typewriter iboon or if the university sent us an inferior photocopy

Previously copyrighted materials (journal articles. published tesis erc) are not filmed

Reproducion in full or in part of this film is governed by the Canadian Copyright ACl, R S C 1970. C C-30

\section{THĖSES CANADIENNES}

\begin{abstract}
AVIS
La qualite de cette microfiche depend giandement deta quakt de la inese soumise au microfilmage Nous avons tous larl pou assurer une qualite superieure de reproduction

S'll manque des pages. veuillez communiquer avec luniver sité qui a contêré le grade

La qualité dimpression de certaines pages peut laisser desirer, surtout si les pages originales oni eté dactylographieb a l'aude diun rutan use ou si runiversile nous a lart parven une photocopie de qualite reterleure

Les documents qui font deja robjet d'un drot d auteur (anticte de revue, examens publies, etc) ne sont pas microfitmes

La reproduction. meme partielle. de ce microfilm est soumis a la Lor canadrenne sur le droit d auteur, SRC 1970, c C-3C

\author{
LA THESE A ETE \\ MICROFILMEE TELLE QUE \\ NOUS L'AYONS RECUE
}

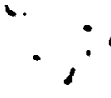

THIS DISSERTATION HAS BEEN MICROFILMED EXACTLY AS RECEIVED 


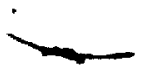

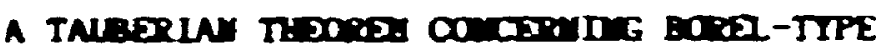

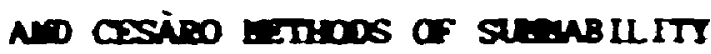

Dy

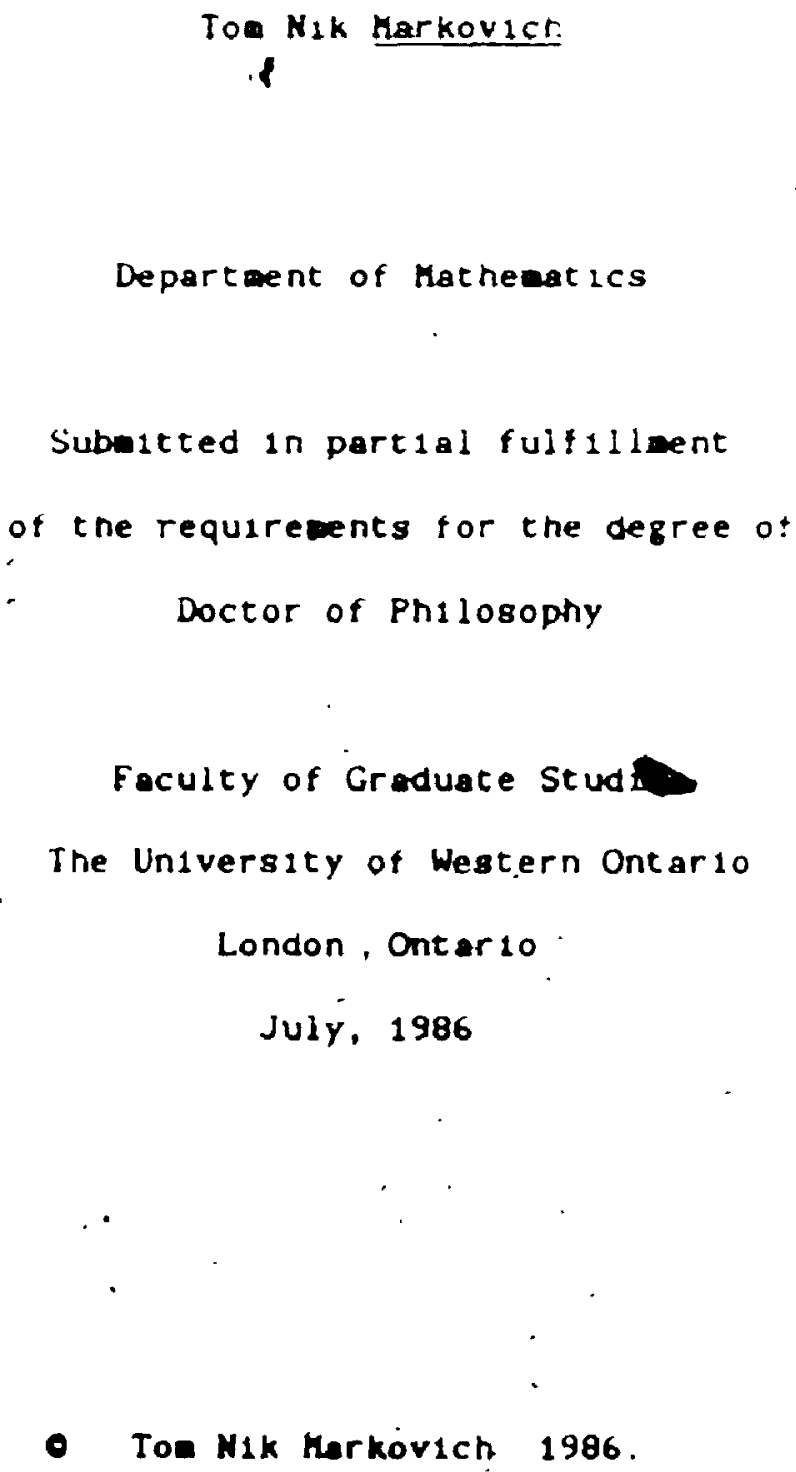

o 


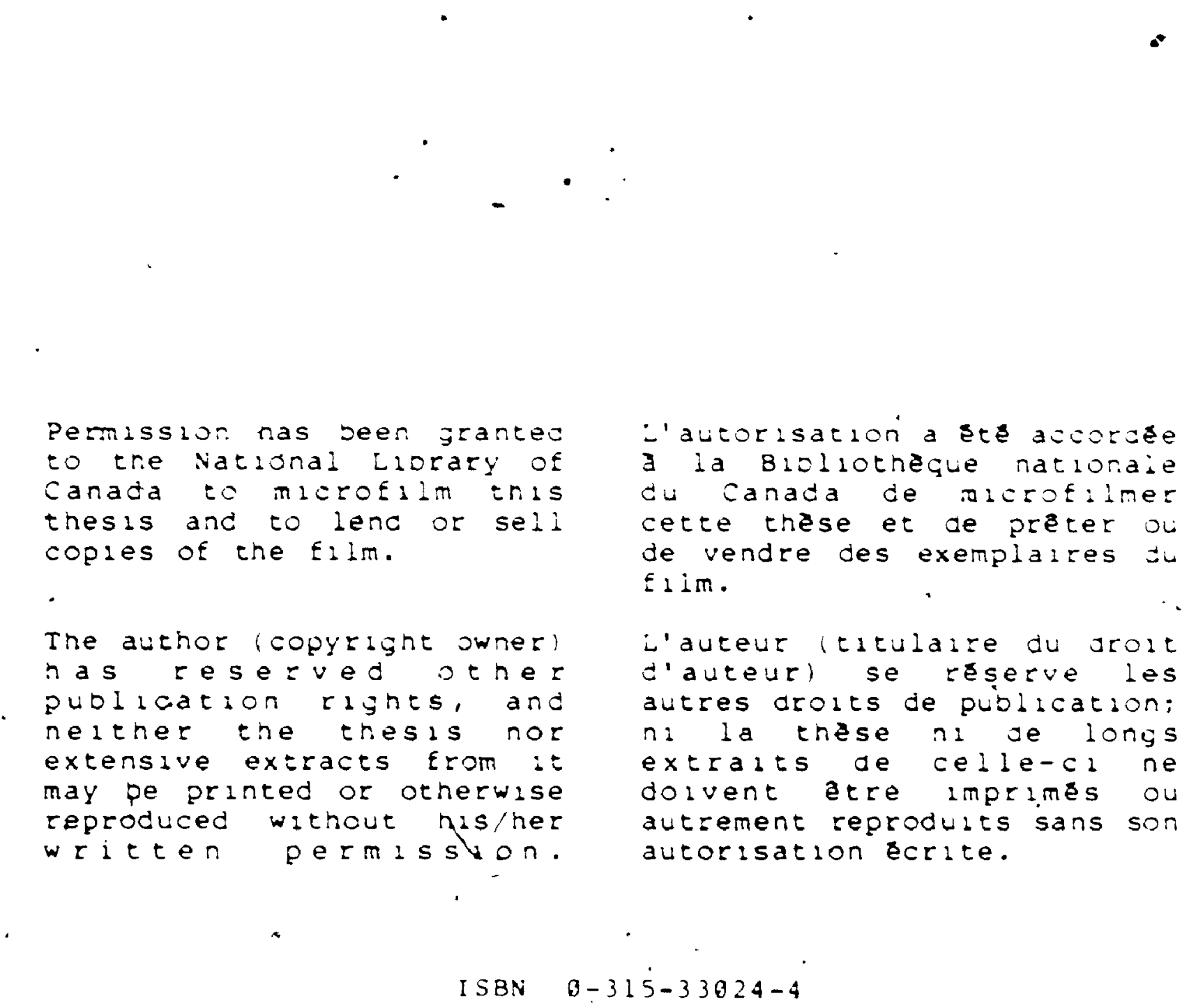

Pemissior. nas veen granted to tre vational Liorary of Canada to microfilm tnis thesis and to lenc or seli copies of the film.

The author (copyright swner) has $r$ esezued other publication rights, and nelther the thesis nor extensive extracts from it may pe printed or otherwise reproduced without his/her writen permisston. -'autorisation a ste accorcese 3 la Bisliotheque nationaie cu canada de alcrotimer cette these et de preter ou de vendre des exemplaires du Elim.

i'auteur (tritulare du arolt d'auteurl se reserve les autres droits de publication; ni la these ni de longs extralts de celle-cl ne dolvent etre imprimes ou autrement reprodults sans son autorisation ecrite.

\section{ISBN $\quad 0-315-33024-4$}




\section{ÁBSTRACT}

Let $r>0, a>0, a N_{0}+\beta>0$ where $N_{0}$ is a non-negat ive integer. and let $s_{n}=\sum_{0}^{n} a_{k}$. The Borel-typessuability aethod $(B, a, \beta) 1 \mathrm{~s}$ defined as follows: $s_{n} \rightarrow \ell(B, \alpha, \beta)$ or $\sum_{0}^{\infty} a_{n}=\ell(B, \alpha, \beta)$

$$
\text { 1t } \dot{a t}^{-x} \sum_{N_{0}}^{\infty} s_{n} \frac{x^{a n+\beta-1}}{\Gamma(a n+\beta)} \rightarrow l \text { as } x \rightarrow \cdots \text {. }
$$

The special case'(B,1,1) is the ordinary Borel exponential wethod $B$.

The wain result of this thesis is the following Tauberian theored for subality $(B, \alpha, \beta)$ :

If $\left\{s_{n}\right.$. 18 a real sequence that satisfles the slouly decreasins condition (Tr): $\lim _{\delta \rightarrow 0+} 11 \pm \lim _{n \rightarrow \infty} \lim _{n+\delta / n} \frac{g^{-8} n}{n^{r}}, 0$ : and $s_{n} \rightarrow \ell(B, \alpha, \beta)$, then $s_{n} \rightarrow \ell\left(C_{2 r}\right)$. Here $C_{2 r}$ denotes 2 he Cesaro nethod of order $2 r$.

In particular, if $a_{n}=Q_{2}\left(n^{r-1 / 2}\right), n=1,2 \ldots$. then ( $T_{r}$ ) jolds and thus the result extends a cheorea due to Kuet.

It 18-ghown, by a Vijayaraghavan type theorea, that ( $T_{r}$ ) and $s_{n} \rightarrow\left\langle(B, a, \beta)\right.$ together Iaply that $s_{n}=$ Orn'). Next a known bethod of sumability $V_{\alpha} 18$ conidered. Th1s 18 a specilil case of the Valiton method and ti is shoin that mability $(B, \alpha, \beta)$ is equivalent to oumability $y_{a}$ provided thet $a_{n}=0\left(x^{F}\right)$. The degired Tauberian theored 18 thẹn deduced fron established results. 


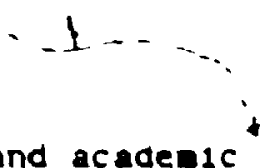

I would like to thank Prof. D. Borvein for his advice and acadenct guidance during ay years at Heatern.

1 

The author of this thesis has granted The University of Western Ontario a non-exclusive license to reproduce and distribute copies of this thesis to users of Western Libraries. Copyright remains with the author.

Electronic theses and dissertations available in The University of Western Ontario's institutional repository (Scholarship@Western) are solely for the purpose of private study and research. They may not be copied or reproduced, except as permitted by copyright laws, without written authority of the copyright owner. Any commercial use or publication is strictly prohibited.

The original copyright license attesting to these terms and signed by the author of this thesis may be found in the original print version of the thesis, held by Western Libraries.

The thesis approval page signed by the examining committee may also be found in the original print version of the thesis held in Western Libraries.

Please contact Western Libraries for further information:

E-mail: libadmin@uwo.ca

Telephone: (519) 661-2111 Ext. 84796

Web site: http://www.lib.uwo.ca/ 


\section{Cingerso}

\section{nm:ooction}

\section{(1) Definitions and diecuanion.}

Let $\left\{s_{n}\right\}$ be a sequence of complex numers. If a given athod of sumabll1ty $P$ aes1gns a "Ile1t" $l$ to the paquence $\left[g_{n}\right.$ ) we say that 1 t 18 liatable $P$ to $l$ and urite $s_{n} \rightarrow l(P)$. In the case of series $\delta$ of complex terms. If $P$ assigns "sun" $l$ to $\sum_{0}$ " we say that it ia sumable $P$ to $l$ and write $\sum_{0}^{\infty} a_{n}=l(P)$.

The wethod $P$ 18 said to be regular if $s_{n} \rightarrow l$ os $n \rightarrow * 1$ inplies $a_{n} \rightarrow \ell(P)$. Necessary and suffictent conditions for regularity of a wide class of sumability nethods can be found in Hardy([12], Chapter 3 ).

Assume that we nou have two nethods of gumablitty $P$ and $Q$. We say that $Q$ includes $P$ if $s_{n} \rightarrow l(P) \Rightarrow s_{n} \rightarrow l(Q)$, and that $P$ and $Q$ are equivalent if $s_{n} \rightarrow \ell(P) \rightarrow s_{n} \rightarrow \ell(Q)$.

Suppose $l, a_{n}(n=0,1,2, \ldots)$ are arbltrary complex numbers, and that a>0, $x>0$. Furtherwore, suppase $B \in R$ and $N_{0} 18$ non-negat ive integer such that $\alpha N_{0}+\beta>0$. Let $g_{n}=\sum_{\delta}^{n} a_{r} \cdot n_{-1}=0: l_{N_{0}}=l-B_{N_{0}-1}$ ond define Borel-type sums $a, \beta$ and ${ }^{8} \alpha, \beta$ by

$$
\theta_{\alpha, \beta}^{(x)}=\sum_{n=N_{0}}^{\infty} \frac{x^{\alpha n+\beta-1}}{\Gamma(a n+\beta)} \quad ; \quad s_{\alpha, \beta}(x)=\sum_{n=N_{0}}^{\infty} \frac{x_{n}^{a n+\beta-1}}{\Gamma(a n+\beta)}
$$

Borel-type wans are defined by

$$
A_{\alpha, \beta}(x)=\int_{0}^{x} e^{-t} a_{\alpha, \beta}(t) d t \quad ; \quad s_{\alpha, \beta}(x)=\alpha e^{-x} a_{\alpha, \beta}(x) .
$$


Borel-type athods are defined as follows:

\section{(A) Sumadility:}

$$
\begin{aligned}
& \text { (1) } s_{n} \rightarrow \ell\left(B^{\prime}, \alpha, \beta\right) \text { if } A_{\alpha, \beta}(x) \rightarrow \ell_{N} \text { as } x \rightarrow \cdots \\
& \text { (11) } s_{n} \rightarrow \ell(B, \alpha, \beta) \text { if } s_{a, \beta}(x) \rightarrow \ell \text { as } x \rightarrow \cdots
\end{aligned}
$$

The sumability ethods $(B, 1,1)$ and $(B, .1,1)$ are the ordinary Borel exponent 191 and Borel integrgl wethods isee Hardy (12)). The $(B, a, \beta)$ and $\left(B^{\prime}, a, \beta\right)$ methods wefe introduced by Borvein in $(4)$. A further discussion of these methods can also be found in $[S],[6\}$, and $[7]$.

The Cesaro ethod 19 probably the cost well-known sumability method. For $p>-1$, the sequence $\left[s_{n}\right\}$ is lialtable in the Cesaro sense, written $s_{n} \rightarrow \ell\left(C_{p}\right)$, if

$$
\begin{aligned}
& c_{n}^{(p)}=\frac{s_{n}^{(p)}}{A_{n}^{(p)}} \rightarrow e \text { and } n \text {, where } A_{n}^{(p)}=\left(\begin{array}{c}
n+p \\
n
\end{array}\right) \text { and } \\
& s_{n}^{(p)}=\sum_{v=0}^{n} A_{n-v}^{(p-1)} \otimes_{v} \cdot C_{n}^{(p)} \text { ionowled the p-th Cesaro neer of }\left[s_{n}\right\} \text {. }
\end{aligned}
$$

A discussion of these methods can be found in Hardy (12).

The ain reoult of this thesis is a theorea which states that ${ }_{n} \rightarrow \underline{L}\left(C_{2 r}\right)$ if $8_{n} \rightarrow\left\langle(B, \alpha, \beta)\right.$ and $\left[\theta_{n}\right\}$ satisfies a condition (called

- Tauberien condicions involving a fixed non-negative real number $r$.

In eneral neither tumabllity $(B, \alpha, \beta)$ nor sumability $C_{p}$ includes 
the other. We now give examples which"show that nelther of these Inclusions 18 true in general. Consider the divergent serzes ([12), p. 2(3)

$\sum_{n=1}^{\infty} a_{n}=\sum_{n=1}^{\infty} n^{a-1} \exp \left(A 1 n^{a}\right)$ where $A>0$ and $0<a</ 2$. It 18 clear that $a_{n}=O\left(n^{-1 / 2}\right)$. By the Borwein and Robinson Tauberiln theorea $[7\}$ the series $\sum_{n=1}^{\infty} a_{n} 19$ not sumable $(B, \alpha, \beta)$ for any a ana $\beta$. But by a result due to Hardy and Littlewood ([127. Theoren 84 with $B=1-8)$ the series $\sum_{n=1}^{\infty} a_{n} i s$ sumable $c_{p}$ for every $p$, 0 . This ekapple shows

- Epat the $(B, \alpha, \beta)$ nethod does not include the $C_{p}$ wethod. On the other hąnd, jet $a>1$ and consider the series

$\sum_{n=0}^{\infty} a_{n}=\sum_{n=0}^{\infty}(-a)^{n}$. It 18 known (see Hardy $(12)$, p. 80) that $\sum_{n=0}^{E}(-a)^{n}=\frac{1}{1+a}(B)$. Now for any norrnegat 1ve integer $n>-\beta / a$, the $\sum_{n=n}^{\infty} \frac{(-a)^{n}}{\Gamma(\alpha)+\beta)} x^{n}$ converges for all $x$, and hence by a reault due to Borwe in ([5], Result (I) $\sum_{n=0}^{\infty}(-\infty)^{n}=\frac{1}{1+a}(B, \alpha, \beta)$ where 1 , $\left.\alpha\right) 0$ and $\dot{B} 10$ real. However, alnce $a_{n}=(-a)^{n}$ is not $O\left(n^{P}\right)$ for any $p$ it follows fros the jidattation theores for Cesaro sumablity 1 wee Hardy. 
(12). Theoree 46) that $\sum_{n=0}^{\infty}(-2)^{n} 18$ not sumable $c_{p}$ for any $p$. Thus, the $C_{p}$ eethod does not include the $\left.\beta, \alpha, \beta\right)$ nethod.

Let $r$ be any fixed non-negiative number, and let $\{8\}$ be a sequence of real numbers. We consider the following (Tduberian) condition

$\left(T_{r}\right): \quad \lim _{\delta \rightarrow 0+} \lim _{n \rightarrow \infty} \operatorname{lnf} \operatorname{mox}_{n+\delta / n} \frac{8 m^{-s_{n}}}{n^{r}}, 0$

Clearly if $\left[g_{n}\right]$ is an increasing sequence then the condition '( $T_{r}$ ' is setisfied. We will discuss the condition $\left(T_{r}\right.$ ' at greater length in Chapter 2, and in particular we show there, that if $c_{n}:=s_{n}-s_{n-t}>-k n^{r-1 / 2}, n=1,2, \ldots(k 18$ a constant) then (T, nolds.

A condition of the form $\left(T_{r}\right)$ is often called a slowly decreasing condition (spe $(12)$ or $(19))^{\circ}$

The and reqult of thig thesis as the following Tauberian theorea: If $s_{n} \rightarrow \ell(B, a, B)$ and $\left(T_{r}\right)$ holds, then $s_{n} \rightarrow \ell\left(C_{2 r}\right)$. This 18 Theoree (4.2) of the thesis; see the end of thio introduction for the sethod of nubering.

We shall also be concerned with the circle fanly of sumability ethods consiating of the Euler, Borel, Meyer-Kön18. Taylor and Valiron sethode. 
(1) For $\alpha>0$. The sequence $\left\{s_{n}\right\} 18$ sald to converge to $l$ in the sense of the Valiron method of sumability with parameter a if $\sqrt{\frac{a}{2 \pi n}} \sum_{1=0}^{\infty} s_{1} \exp \left\{-\frac{a}{2} \frac{(1-n)^{2}}{n}\right\} \rightarrow l$ as $n \rightarrow-, n=1,2, \ldots$, we then

Wr:te $\left.s_{n} \rightarrow u v_{a}\right)$

The Borel exponential wethod $B$ has already been mentioned but for completeness we shall define it here.

(11) $s_{n} \rightarrow \ell(B)$ weans $\sum_{1=0}^{\infty} s_{1} \frac{e^{-x} x^{1}}{1 !} \rightarrow l$ as $x \rightarrow \cdots$.

(111) The Euler aetrod $E_{\alpha^{\prime}} \cdot \alpha \in(0,1)$. for which

$$
s_{n} \rightarrow \ell\left(E_{\alpha}\right) \operatorname{eeans} \sum_{l=0}^{n}\left(\begin{array}{l}
n \\
1
\end{array}\right) \alpha^{1}(1-\alpha)^{n-1} s_{1} \rightarrow l \text { as } n \rightarrow \cdots
$$

(1v) The Keyer-konts netrod $S_{\alpha}$, a $E(0,1)$, for which

$$
s_{n} \rightarrow r\left(s_{\alpha}\right) \text { means }(1-\alpha)^{n+1} \sum_{1=0}^{\infty}\left(\begin{array}{c}
n+1 \\
1
\end{array}\right) \alpha^{1} s_{1} \rightarrow \ell \text { as } n \rightarrow \bullet
$$

(v) The Taylor method $T_{\alpha}, \alpha \in(0,1)$, for which

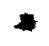

$$
s_{n} \rightarrow \ell\left(T_{\alpha}\right) \text { means }(1-a)^{n+1} \sum_{1=0}^{\infty}\left(\begin{array}{c}
n+1 \\
1
\end{array}\right) \alpha^{1} s_{n+1} \rightarrow l e a n \rightarrow \cdots
$$

For comparison purposes; Hardy ( (12), Chepter 9), definea sumability $(e, c), c>0$, for wh1ch, $n \rightarrow l(e, c)$ wean

$$
\left(\frac{c}{n n}\right)^{1 / 2} \sum_{n=-n}^{\infty} a_{n+h} \exp \left(-c \frac{t^{2}}{n}\right) \rightarrow l \infty n \rightarrow \cdots
$$


Therefore the ta method of sumablitt ls the (e, a 2 ) wethod ot sumabld1t?

We now state sat theorems and show how our wain result is

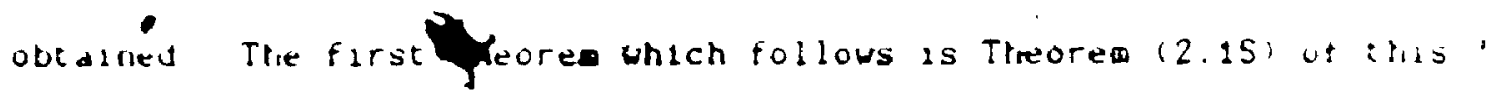
inesls, the secogd is Theorea $(3.21)$, and the others are known

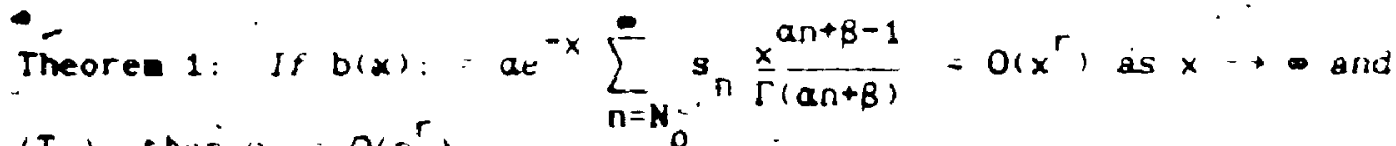
$\left(T_{r}\right)$, cheris ${ }_{n}=O\left(n^{r}\right)$ 1.

Theoree 2: If $s_{n}-O\left(n^{r}\right)$ then $s_{n} \rightarrow \ell(B, a, \beta) \rightarrow s_{n} \rightarrow \ell\left(v_{a}^{\prime}\right.$.

Theores 3: (Faulhaber [10) or Bingham (2)) If $s_{n}=O\left(n^{r}\right.$ ) and $0<a<1$. 'then $\left.s_{n} \rightarrow \ell \cdot S_{a}\right) \rightarrow s_{n} \rightarrow\left(i v_{1-a}\right)$

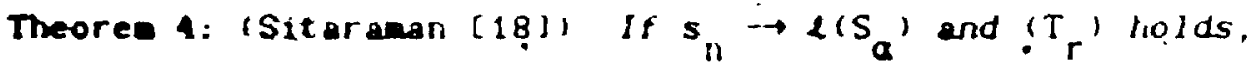

then $s_{n} \rightarrow l+C_{2 r}$

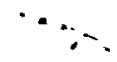

Theorea 5:: (Borwe in $[S]$ ) If $\alpha>Y>Q_{1}$ and for any non-negal ive' integer $\mathrm{H}>-\delta / \gamma$.

$\sum_{n=1}^{\infty} \frac{n}{\Gamma(r n+\delta)} x^{n}$ is convergent for all $x$, then

$$
s_{n} \rightarrow \ell(B, a, \beta) \bullet B_{n} \rightarrow \ell(B, \gamma, \delta)
$$


The proaf of our main result is as follows:

$s_{n} \rightarrow(B, a, B)$ toplies that $b(x)=O\left(x^{r}\right.$, as $x \rightarrow$ and lience, br

Theored 1, that $s_{n}=0 i n^{r}$, Theorem 2 now shows that $s_{n} \rightarrow l\left(V_{a}\right.$ '

whle theored 5 shows that there is no loss in generallty in waking

the restriction 0 , a : It then follows, by Theorem 3, that

$s_{n} \rightarrow 2\left(s_{1-a}\right.$ ' and hence, br Theored 4, that $s_{n} \rightarrow 2\left(C_{2 r}\right.$ '.

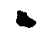

-The special case $\alpha-\beta=1, r=0$ of Theorem (4.2) with $+T_{0}$ ) repiaced by ${ }_{n}=O\left(n^{-1 / 2}\right.$, 18 the original O-Tauberian theorem fur Burel summablity due to Hardy and Littlewood [13]. The Borel sumabllity case $\alpha-\beta-1, r, 0$ of Theorem (4.2) has been proved by Rajagopal (17), and the corresponding theorem vith smability $(B, a, B)$ replaced by sumability $S_{\alpha} 1 s$ due to Sitaraman (18). More recently Bingham (3) proved the theorem with sumability $(B, a, B)$ replaced by sumagility methods of random walk-type (see belou for definitions). As for the general $(B, \alpha, B)$ method, the case $r \neq 0$ of theored $(A, 2)$ with $\left(T_{r}\right.$ ) replaced by $a_{n}=0\left(n^{r-1 / 2}\right) 18$ due to Borve $1 n(b)$, and the case $c=0$ with $\left(T_{0}\right)$ replaced by $a_{n}=O\left(n^{-1 / 2}\right)$ is due to Borvein and Robsison [7]. The most general result to-date for the $(B, \alpha, \beta)$ method 1s due to kwee [15] who proved the case of Theorea (4.2) w1th (T, replaced by $a_{n}=O\left(n^{r-1 / 2}\right)$.

We now define sumability methods of the randon walk-type. Let P be a probability law with sean $p$ ahd variance $\sigma^{2}$. Let $x_{1} x_{1}, x_{2}, \ldots$ be - sequence of Independent and Identically distributed randon variables 

As an example, let $P$ be the Bernoulij lau with paraneter a $\in(0,1)$. Then $P(x=1)=a, P(x=0)=1-a$, and the generat 1 ng function $C$ is glven by $G(s)=1-a+s a$. Since $G^{n}(s)=\sum_{k=0}^{n}\left(\begin{array}{l}n \\ k\end{array}\right) a^{k}(1-\alpha)^{n-k} s^{k} d t$ follows that $P\left(\dot{S}_{n}=k\right)=\left(\begin{array}{l}n \\ k\end{array}\right) a^{k}(1-a)^{n-k}$, Thus with the Bernould lau $P$ $s_{n} \rightarrow \ell(P)$ is $s_{n} \rightarrow \ell\left(E_{\alpha}\right)$.

In the case where $P$ is the geowetric law with parameter a $\in(0,1)$ we have that $P(X=k)=(1-a) a^{k}, k=0,1 \ldots ;$ and hence that $P\left(S_{n}=k\right)=\left(\begin{array}{c}n+k-1 \\ k\end{array}\right) a^{k}(1-a)^{n}$ (see $\left.(11\}, p \cdot 217\right)$. It follows that $s_{n} \rightarrow \ell(P)$ is $(1-a)^{n} \sum_{k=0}^{\infty}\left(\begin{array}{c}n+k-1 \\ k\end{array}\right) a^{k} s_{k} \rightarrow \ell$ as $n \rightarrow \cdots$. and this is equivalent to $s_{n} \rightarrow \ell\left(s_{\alpha}\right.$ ).

The $(B, \alpha, \beta)$ etrod of summability is in fact equivalent to a special case of a very general type of sumabifity bethod, usually known as the $J$ method or Integral function method.

Let. $J(z)=\sum_{n=0}^{\infty} J_{n} z^{n}$, where $j_{n}>0$ for $n>0$, and $J 18$ an entire function but not a polynomal. Then $a_{n} \rightarrow \ell(J)$ weans $\sum_{n=0}^{\infty} a_{n} J_{n} x^{n}-(J(x)$ as $x-+\cdots$. Since $\sum_{n=N_{0}}^{\infty} \frac{x^{\alpha n+\beta-1}}{r(a n+\beta)} \sim a^{-1} e^{x}$ es $x \rightarrow \cdots$. isee Borve1n (5), p. 130 , with $\delta=\beta-1$, at follows that the $(B, \alpha, \beta)$ eethod a equivalent to tho $J$ method vith

$$
J_{n}=\left\{\begin{array}{cl}
\frac{1}{\Gamma(\alpha+\beta)} & , n>N_{0} \\
0 & , 0<n<N_{0} .
\end{array}\right.
$$


Then $x^{\beta-1} \sum_{n=0}^{\infty} J_{n} x^{a n}-\alpha^{-1} e^{x}$ as $x \rightarrow \cdots$. Lett: ns $y=x^{a}$ ve obtain $y^{\frac{\beta-1}{\alpha}} \sum_{n=0}^{\infty} J_{n} y^{n}-\alpha^{-1} e^{\frac{1}{\alpha}}$ as $y \rightarrow$, i.e. $x^{\frac{\beta-1}{\alpha}} J(x)-\alpha^{-1} e^{x^{\frac{1}{\alpha}}}$ as $x \rightarrow \bullet$

Hence:

$$
\frac{1}{J(x)} \sum_{n=0}^{\infty} 8_{n} y_{n} x^{n}-\alpha e^{-x^{\frac{1}{\alpha}}} x^{\frac{\beta-1}{\alpha}} \sum_{n=0}^{\infty} 8_{n} 3_{n} x^{n}=a e^{-u} \sum_{n=N}^{\infty} s_{n} \frac{u^{\alpha n+\beta-1}}{\Gamma(a n+\beta)}
$$

where $u=x^{1 / Q}$. Since the $J$ methods are regular (see Hardy [12], Theorea 33) it follows that sumability (B,A,B) is also regular.

Borwein([S], III and IV) has proved the following result:

Let $h$ be an analytic function of $z=x+1 y$ in the region $x>x_{0}$.

and suppose that

(1) when $x>x_{0}$ and $|z|$ is large

$h(z)=z^{a z+B} e^{Y z}\left\{c+o\left(\frac{1}{T z}\right)\right\}$, where $c>0, \alpha>0, B, Y \in R$ and

(11) $h(x) 18$ real for $x>x_{0}$.

Let $J(z)=\sum_{n=N_{0}}^{\infty} \frac{z^{n}}{n(n)}$.

Then $o_{n} \rightarrow \ell(J) \sim 8_{n} \rightarrow \ell(B, \alpha, \beta+1 / 2)$.

In particular, taking $h(z)=(r(a z+b))^{c}(z+p) q e+r$ where $b, c, p, q$, $r \in R, e>0$ and $a c+q>0$ so that

$$
J(z)=\sum_{n=N_{0}}^{\infty} \frac{z^{n}}{\left.[r(a n+b)]^{c}(n+p)\right)^{q n+r}}
$$


$s_{n} \rightarrow e(j)-s_{n} \rightarrow e(B, a c+Q, D c+r-c / 2+1 / 2)$.

It follows that Theorea (4.2) is in fact true fur quite $n$ vide clasy $n$

of sumability nethods. Theoree (A.2) reasing true it $s_{n} \rightarrow(B, a, \beta)$ is replaced by $s_{n} \rightarrow \ell\left(B^{\prime}, \alpha, \beta^{\prime}\right.$. This 13 a consequence of the tollowing known result, Borwein ([4] Theorea 2 ):

$s_{n} \rightarrow \ell(B, G, B+1) \cdots s_{n} \rightarrow \ell\left(B^{\prime}, Q, B\right)$.

(b) Strong sumabifity with index $p>0$.

(1) $s_{n} \rightarrow \ell\left[B^{\prime}, \alpha, \beta\right]_{p}$ if $\int_{0}^{x} e^{t}\left|A_{\alpha, \beta-1}(t)-l_{N_{0}}\right|^{p} d t=o\left(e^{x}\right)$ as $x \rightarrow$. $(11) s_{n} \rightarrow \ell(B, \alpha, \beta)_{p}$ if $\int_{0}^{x} e^{t}\left|s_{\alpha, \beta-1}(t)-l\right|^{p} d t=o\left(e^{x}\right)$ as $x \rightarrow \infty$.

These strong methods of sumability were introduced by Borwein and Shawyer in [9]. Varlous theoreas linking gtrong gumabillty with sumabllity $(b, \alpha, \beta)$ are knoin (see $(8)$, and $(9))$. For example in $([8]$, Theorea 3$) s_{n} \rightarrow \ell[B, \alpha, \beta]_{1} \otimes_{n} \rightarrow \ell(B, \alpha, \beta)$ and so strong sumab1l1ty $(B, \alpha, B]_{1}$ can replace the hypothesis of sumaabllity $(B, \alpha, \beta)$ in Theoren $(4.2)$.

\section{(2) Bechod of Nubering}

The thesis conlaine lhis introduction and four chaptera. Theorems, corollerles, lemas, remarks, and equations to whact we will later refer are numbered consecutively within each chapter and placed in the argin for ease of reference. Thu in chapter three. 


\section{CHATER 1}

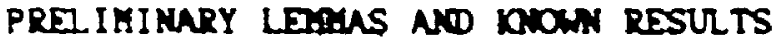

Our ald in this chapter 18 to state certain regultg which are * needed in later chapters.

(1.1) Lem: If $f(x)=\sum_{n=0}^{\infty} a_{n} x^{n}$ has radius of convergence $R$, 0 ,

then for $|x| 6 r\left(R, f(x)=\sum_{n=0}^{m} a_{n} x^{n}+0\left(|x|^{m+1}\right)\right.$.

Proof: $\left|f(x)-\sum_{n=0}^{m} a_{n} x^{n}\right|+\sum_{n=\infty+1}^{\infty}\left|a_{n}\right||x|^{n}=|x|^{\infty+1} \sum_{n=\infty+1}^{\infty}\left|a_{n}\right||x|^{n-\infty-1}$

$\left.\left.|| x\right|^{n+1} \sum_{n=\infty+1}^{\infty} a_{n}\left|r^{n-m-1}=n_{r}\right| x\right|^{m+1}$, since the ser 1 es convergea.

The leme follows. //

(1.2) Reark: It 19 vell known that for $|x|<1$.

$\log (1+x)=\sum_{n=1}^{\infty} \frac{(-1)^{n-1}}{n}-x^{n}$ and $(1+x)^{k}=\sum_{n=0}^{\infty} \cdot\left(\begin{array}{l}k \\ n\end{array}\right) x^{n}$

Hence by Lema (1.1)

$$
\begin{aligned}
& \log (i+x)=\sum_{n=1}^{D} \frac{(-1)^{n-1}}{n} x^{n}+0\left(|x|^{-1}\right) \text { and } \\
& (1+x)^{k}=\sum_{n=0}^{D}\left(\begin{array}{l}
k \\
n
\end{array}\right) x^{n}+0\left(|x|^{n+1}\right) \text {, for }|x| 6 r<1 .
\end{aligned}
$$


14

(1.3) Lean: Let be a function unset decreases on $\left(n_{0}, n_{1}\right)$. Then

$$
\sum_{n=n_{0}}^{n}(n) \cdot \int_{n_{0}}^{n_{1}}(t) d t+\phi\left(n_{0}\right) .
$$

Proof: Since is decreasing on $\left[n_{0}, n_{1}\right]$ we have:

$$
\int_{n-1}^{n}(t) d t>(n), n=n_{0}+1, n_{0}+2, \ldots, n_{1} .
$$

Thus, $\left.\int_{n_{0}}^{n_{1}} \phi(t) d t=\sum_{n=n_{0}+1}^{n_{1}} \int_{n-1}^{n} \phi(t) d y\right) \sum_{n=n_{0}+1}^{n_{1}} \phi(n)=\sum_{n=n_{0}}^{n_{1}} \phi(n)-\Delta\left(n_{0}\right)$.

The result follows. $/ / /$

(1.4) Lea: Let $(t)>0$ an $\left(n_{0}, n_{1}\right)$, Increasing on $\left[n_{0}, x_{0}\right]$ and decreasing on $\left[x_{0}, n_{1}\right]$. Then for $\left.n_{1}\right) x_{0}+1$,

$$
\sum_{n=n_{0}}^{n_{1}}+(n) \cdot \int_{n_{0}}^{n_{1}} p(t) d t+\varphi\left(x_{0}\right)
$$

Proof: Let $8_{0}$ denote the integer part of $x_{0}$. If $n_{0}<s_{0}<x_{0}<s_{0}+1<n_{1}\left(t h e\right.$ case $n_{0}=s_{0}$ is immediate), then

$$
\begin{aligned}
& \sum_{n=n_{0}}^{x_{0}-1} \phi(n), \sum_{n=n_{0}}^{s_{0}-1} \int_{n}^{n+1} p(t) d t=\int_{n_{0}}^{8_{0}} \phi(t) d t \text {, } \\
& \sum_{n=s_{0}+2}^{n_{1}}(n)=\sum_{n=\infty_{0}+1}^{n_{1}-1}(n+1) \cdot \sum_{n=s_{0}+1}^{n_{1}-1} \int_{n}^{n+1}(t) d t=\int_{0_{0}+1}^{n_{1}}(t) d t
\end{aligned}
$$


Thus,

(1.5) $\sum_{n=n_{0}}^{n_{1}} \phi(n)=: \sum_{n=n_{0}}^{8_{0}^{-1}} \phi(n)+\left(8_{0}\right)+\left(s_{0}+1\right)+\sum_{n=8_{0}+2}^{n_{1}} \phi(n)$

$\int_{n_{0}}^{n_{1}}(t) d t+\left(8_{\Omega}\right) \cdot\left(s_{0}+1\right)-\int_{s_{0}}^{s_{0}+1} \bullet(t) d t$

Now consider the two cases: (1) $\bullet\left(s_{0}\right)\left(\bullet\left(s_{0}+1\right)\right.$ and $\left.(11) \bullet\left(s_{0}\right)\right)\left(s_{0}+1\right)$.

If (1) holds, then $\left(s_{0}\right)\left(\phi(t)\right.$ for all $t \in\left[s_{0}, s_{0}+1\right)$, so that

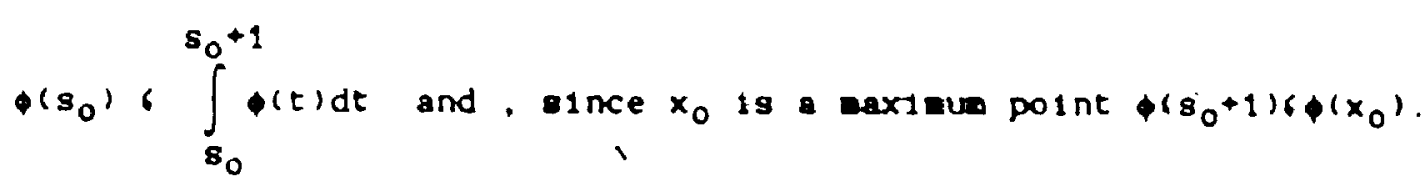

Thus, $\left(s_{0}\right)+\left(s_{0}+1\right), \int_{8_{0}}^{s_{0}+1}-(t) d t+\phi\left(x_{0}\right)$

If (11) holds, then $\left(8_{0}+1\right), \int_{8_{0}}^{s_{0}^{+1}}(t) d t$ and, since $x_{0} 19$ a axinum point $\left(8_{0}\right) 6\left(x_{0}\right)$.

Thus, $\left(8_{0}\right)+\left(s_{0}+1\right), \int_{8_{0}}^{8_{0}^{+1}}(t) d t+\left(x_{0}\right)$. So in case (1) or $(11),\left(8_{0}\right)+\left(8_{0}+1\right)-\int_{s_{0}}^{s_{0}^{+1}}(t) d t, t\left(x_{0}\right)$.

The result now follows froe (1.5). $/ 1 /$

$\because$

Let us now $f 1 x$ sose notation which will be used

thesis. Set $n_{n}=n-x / \alpha$ and $k_{n}=-n_{n}$.

The next feu leman will be ued to obtain estiantes (when $x \rightarrow-$ )

for sum of the form:

$\sum_{n=n_{0}}^{n_{1}} n_{n}^{c} \exp \left(-\frac{a}{x} n_{n}^{2}\right), \sum_{n=n_{0}}^{n_{2}} k_{n}^{c} \exp \left(-\frac{2}{x} k_{n}^{2}\right)$, where, 0 and $c, 0$. 
(i.6) Lame: Let $f$ be decreasing function on $\left[h_{n_{0}}, h_{n_{1}}\right]$. Then,

$$
\sum_{n=n_{0}}^{n_{1}} f\left(n_{n}\right), f\left(n_{n_{0}}\right)+\int_{n_{n_{0}}}^{n_{n_{1}}} f(y) d y .
$$

16

The result an be obtained directly as in Lems (1.3), however, we choose to deduce the result.

1

Proof: Define by $(t)=f(t-x / a)$, so that $f\left(h_{n}\right)=(n)$. Now $f(t)$ decreasing on $\left[n_{n_{0}}, n_{n_{1}}\right]=\left[n_{0}-x / a, n_{1}-x / a\right]$ is equivalent to (t) decreasing on $\left[n_{0}, n_{1}\right]$.

Thus, by Lea (1.3),

$$
\begin{aligned}
\sum_{n=n_{0}}^{n_{1}} f\left(n_{n}\right)=\sum_{n=n_{0}}^{n_{1}}(n) & \int_{n_{0}}^{n_{1}} \phi(t) d t+\phi\left(n_{0}\right) \\
& =\int_{n_{n_{0}}}^{n_{n_{1}}}(y+x / a) d y+\phi\left(n_{0}\right), \text { where } t=y+x / a \\
& =\int_{n_{n_{0}}}^{n_{n_{1}}} f(y) d y+f\left(n_{n_{0}}\right)
\end{aligned}
$$

(1.7) Lame: Let $f$ be a decreasing function on $\left(k_{n_{1}}, k_{n_{0}}\right)$. Then,

$$
\sum_{n \neq n_{0}}^{n_{1}} f\left(k_{n}\right), f\left(k_{n_{1}}\right)+\int_{k_{n_{1}}}^{k_{n_{0}}} f(y) d y .
$$

Proof: Define $g$ by $g(y)=f(-y)$. The function 8 satisfies all the 


$$
\begin{aligned}
& \sum_{n=n_{0}}^{n} f\left(k_{n}\right)=\sum_{n=n_{0}}^{n_{1}} g\left(n_{n}\right) \cdot g\left(n_{n_{0}}\right)+\int_{n_{n_{0}}}^{n_{n}} g(y) d y=f\left(k_{n_{0}}\right)+\int_{k_{n_{1}}}^{n_{n_{0}}} f(y) d y \\
& \therefore
\end{aligned}
$$

In the next lema, $x_{0}$ is not necessarily an integer, hovever, we write $h_{x_{0}}-x_{0}-x / a$ simply to denote the polnt where $f(y)$ attains its maximur : S1milarly, in Lema(1.9), $k_{x_{0}}$ denotes the polnt where $f(y)$ attains its maxioun.

(1.8) Lewa: Let $f(y)>0$ on $\left[h_{n_{0}}, h_{n_{1}}\right]$, increasing on $\left[h_{n_{0}}, h_{x_{0}}\right]$ and decreasing on $\left[n_{x_{0}}, h_{n_{1}}\right\}$. Then

$$
\sum_{n=n_{0}}^{n_{1}} f\left(n_{n}\right), \int_{n_{n_{0}}}^{n_{n_{1}}} f(y) d y+f\left(n_{x_{0}}\right)
$$

Proof: Define by $(t)=f(t,-x / \alpha)$, so that $f\left(h_{0}\right)=\phi(n)$. f(t) increasing on $\left[n_{n_{0}}, n_{x_{0}}\right]$ is' equivalent to $(t)$ increasing on $\left[n_{0}, x_{0}\right]$. and $f(t)$ decreasing on $\left[b_{x_{0}}, h_{n_{1}}\right]$ ls equivalent to $(t)$ decreasing on $\left\{x_{0}, n_{1}\right)$. Horeover, $f(t) \geq 0$ on $\left[n_{n_{0}}, h_{n_{1}}\right] 18$ equivalent to pit)>0 on $\left[n_{0}, n_{1}\right]$.

Thus, $(t)$ gatigfles all the hypotheses of Leme(1.4), and 80

$$
\sum_{n=n_{0}}^{n_{1}} f\left(n_{n}\right)=\sum_{n=n_{0}}^{n_{2}} p(n), \int_{n_{0}}^{n_{1}} \phi(t) d t+\phi\left(x_{0}\right)
$$


18

$$
=\int_{n_{n_{0}}}^{n_{n_{1}}} \phi(y+x / a) d y+\phi\left(x_{0}\right) \text {, where } t=y+x / a \text {. }
$$

$$
=\int_{n_{n_{0}}}^{n_{n_{1}}} f(y) d y+f\left(n_{x_{0}}\right) \cdot 11,
$$

$\checkmark$

(1.9) Lea: Let $f(y) \geq 0$ on $\left[k_{n_{1}}, k_{n_{0}}\right]$, increasing on $\left[k_{n_{1}}, k_{x_{0}}\right]$ and decreasing on $\left[k_{x_{0}}, k_{n_{0}}\right]$. Then

$$
\sum_{n=n_{0}}^{n_{1}} f\left(k_{n}\right) \cdot \int_{k_{n_{1}}}^{k_{n_{0}}} f(y) d y+f\left(k_{x_{0}}\right)
$$

Proof: This 15, of course, Just a relabelling of Lema(1.8).11t

(1.10) Lea: For $a>0, \mu=1,2, \ldots$

- $\quad\left(\frac{a}{2 \pi \mu}\right)^{1 / 2} \sum_{n=0}^{\infty} \exp \left\{-\frac{\alpha}{2}\left(\frac{(a-\mu)^{2}}{\mu}\right\}=1+0(1 / / \mu)\right.$.

Proof: Write $S(\mu):=\sum_{n=0}^{\infty} \exp \left\{-\frac{a}{2} \frac{(n-\mu)^{2}}{\mu}\right\}=\sum_{n=-\mu}^{\infty} \exp \left\{-\frac{a}{2} \frac{n^{2}}{\mu}\right\}$

$$
\begin{aligned}
& =2 \sum_{n=1}^{\infty} \exp \left\{-\frac{a}{2} \frac{n^{2}}{j}\right\}-\sum_{n=\mu+1}^{\infty} \exp \left\{-\frac{a}{2} \frac{n^{2}}{\mu}\right\}+1 \\
& =2 s_{\mu}-T_{\mu}+1 .
\end{aligned}
$$



for all $n>H$

Next,

$$
\begin{aligned}
& |S(\mu)|:=\left\{\frac{a}{2 \pi \nu}\right\}^{ \pm / 2} \sum_{n=0}^{\infty}\left(s_{n}-l\right) \exp \left\{-\frac{a}{2}\left(\frac{n-\mu}{\mu}\right\}\right\} \\
& \text { 6 }\left\{\frac{a}{2 n \mu}\right\}^{1 / 2}\left\{\left(\sum_{n=0}^{n}+\sum_{n=\mu+1}^{\infty}\right\} s_{n}-\left(\exp \left\{-\frac{a}{2}\left(\frac{n-\mu)^{2}}{\mu}\right\}\right)\right.\right. \\
& -s_{1} \cdot s_{2}
\end{aligned}
$$

If $A=\operatorname{arax}_{\operatorname{arn} n} \mid a_{n}-l 1$, then clearly $s_{1}, A\left\{\frac{a}{2 \pi \mu}\right\}^{1 / 2} \sum_{n=0}^{n} \exp \left\{-\frac{a}{2}\left(\frac{n-i)^{2}}{\mu}\right\}\right.$

$$
=\text { o(1) as } \mu \rightarrow \text { : }
$$

- While in $S_{2}$ where $n \partial H+1,1 s_{n}-t+s$ so that

$\left.S_{2}<E\left\{\frac{a}{2 \pi \mu}\right\}^{1 / 2} \sum_{n=n+1}^{\infty} \exp \left\{-\frac{a}{2} \frac{(n-\mu)^{2}}{\mu}\right\}<\epsilon \frac{a}{2 \pi \mu}\right\}^{1 / 2} \sum_{n=0}^{\infty} \exp \left\{-\frac{a}{2}\left(\frac{n-\mu)^{2}}{\mu}\right\}\right.$

$$
=c(1+0(1)) \text { as } \mu \rightarrow \infty \text {. by }
$$

Lenes $(1,10)$.

Thus, $|S(\mu)| 60(1)+\varepsilon(1+o(1))=\varepsilon+o(1)$ as $\mu \rightarrow+0$, arid

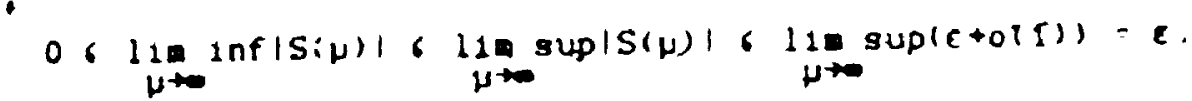

Since $6>018$ arbitrary, we have that $S(\mu)=011$, as $\mu \rightarrow-$.

Therefore, by Leman(1.10),

$$
\left.\left\{\frac{a}{2 \pi \nu}\right\}^{1 / 2} \sum_{n=0}^{\infty} s_{n} \exp \left\{-\frac{a}{2} \cdot \frac{(n-\mu)}{\mu}\right)^{2}\right\}=
$$

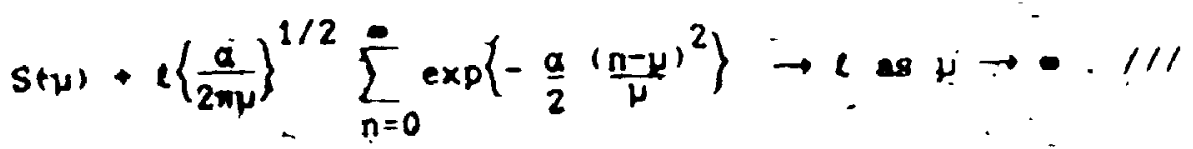




\section{CHAPTIR 2}

\section{A VIJAYNRAGHAVA-TYPE THDORETS}

- In this chapter te show that the conditions ( $T_{r}$ ' and

$$
a_{e}^{-x} \sum_{n=N}^{\infty} s_{n} \frac{x^{a n+\beta-1}}{\Gamma(a n+\beta)}=O\left(x^{r}, \text { as } x \rightarrow \cdots\right.
$$

together idply that $s_{n}=0 i^{r}$, where $r>0$. The dethod of prout is that of Vi)ayaragtavan [19]. In particular te follow Sitaramanc[18],

Lema $S$ and Theores 1 ) who deals with the $S_{a}$ oethod of summabilits since $\frac{q e^{-x} s_{n} x^{a n+\beta-1}}{r(a n+\beta)} \rightarrow 0$ as $x \rightarrow \infty$, it is clear that the actual cholce of $N_{0}$. 15 moterial. We therefore vill assuar that $N_{0}$ satisfles the condition: $N_{0}+B-r>0$, where $r 20$ is some fixed numiter. i.

We also let $c_{n}(x):=\frac{a_{e}^{-\dot{x}} x^{\alpha n+\beta-1}}{-\Gamma\left(\alpha_{n}+\beta\right)}, n>N_{0}$ and $b(x):=\sum_{n=N_{0}}^{\infty} s_{n} c_{n}(x)$. Note that $c_{n}(x)>0$ for $n>N_{0}$ and $x>0$, and that $\sum_{n=N_{0}}^{\infty} c_{n}(x)-1$ as $x \rightarrow \infty$ "see Borveln [S], p. 130 with $8=\beta-1$ ).

The next lema is well known and wlll be used extensively.

(2.1) Leme (Borve $1 \mathrm{n}$ and Robinson (7)). Let $x>0, h_{n}=n-x / a$, $1 / 2<E<2 / 3$, and $0<n<2 E-1$. Then

$$
\begin{aligned}
& e^{-x} \sum_{\ln _{n} \operatorname{lix} E} \frac{x^{\alpha n+\beta-1}}{\Gamma(a n+\beta)}=O\left(e^{-x^{n}}\right) \text {, and } \\
& \frac{a e^{-x}}{\Gamma(a n+\beta)} \frac{x^{\alpha n+\beta-1}}{\Gamma(2 \pi x)} \exp \left(-\frac{a^{-2} n_{n}^{2}}{2 x}\right)\left\{1+o\left(\frac{\left|h_{n}\right|+1}{x}\right)+o\left(\frac{\left|a_{11}\right|^{3}}{x^{2}}\right)\right\} \\
& =\frac{a}{J(2 \pi x)} \exp \left(-\frac{a^{2} h_{n}^{2}}{2 x}\right) \cdot\left\{1+a\left(x^{3 \varepsilon-2}\right)\right\} \text {. If } \mid h_{n} 16 x^{E} \text {. }
\end{aligned}
$$


(2.2) Lewe: $\frac{\left(a_{n}\right)^{r}}{\Gamma(a n+\beta)}-\frac{1}{\Gamma(a n+\beta-r)}$ as $n \rightarrow \cdots$.

Proof: It follows froe Stirling's formula that

$r(a n+\beta)=j(2 \pi) e^{-a n}\left(a_{n}\right)^{a n+\beta-1 / 2}\left\{1+o\left(\frac{1}{n}\right)\right\}$

(see (1), foraula $3,1,18,0.47$ ).

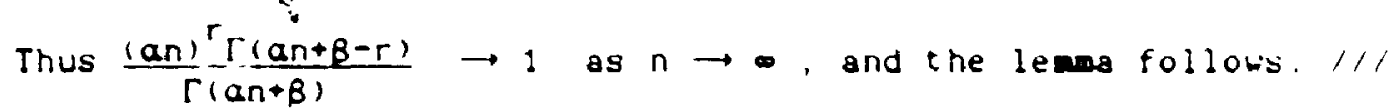

12.3) Leme: Let $1 / 2<5<2 / 3$. Then as $x \rightarrow \infty$

$$
\begin{aligned}
& \sum_{N_{0}\left(n<x / \alpha-x^{E}\right.} n^{r} c_{n}(x)=o(1), \\
& \sum_{n>x / \alpha+x} n^{r} c_{n}(x)=o(1) .
\end{aligned}
$$

Proof: It follows, from Lema (2.2) and Lemma (2.1), that

$$
\begin{aligned}
& \sum_{N_{0} \sigma n<x / \alpha-x^{\varepsilon}} n^{r} c_{n}(x)=\alpha\left\{x^{r} e^{-x} \sum_{N_{0}\left(n<x / \alpha-x^{\varepsilon}\right.} \frac{x^{a n+\beta-r-1}}{\Gamma(a n+\beta-r)}\right\} \\
& =O\left(x^{r} e^{-x^{n}}\right) \quad, \quad 0<n<2 \varepsilon-1 \\
& =o(1) \text { as } x \rightarrow \text {. }
\end{aligned}
$$

The second sum is dealt with giallarly. ///

In the next lease we will be using a slowly decreasing condition of the forn:

$$
\lim _{\delta \rightarrow 0+} \lim _{u \rightarrow \infty} \ln _{u t} \operatorname{lin}_{u+\delta}(u) \frac{s(t)-s(u)}{u^{r}} \geqslant 0 \text {, where }(u)>0 \text {. }
$$

To see what this means in term of $6>0$ consider the following :

let $m_{\delta}(u)=\operatorname{man}_{u \in f u+\delta(u)} \frac{g(t)-g(u)}{u^{r}}$, and $m_{\delta}=110$ inf $a_{\delta}(u)$. 
The slowly decreasing condition above says that

$a_{\delta} \rightarrow$ e say, as $\delta \rightarrow 0+$. Therefore for any $\varepsilon>0$. there exista $\delta_{0}=\delta_{0}(\varepsilon)>0$ such that $\delta_{\delta}-,-\varepsilon / 2$ whenever $0<\delta_{6} \delta_{0}$.

In particular $\mathbf{a}_{\delta_{0}}>-\varepsilon / 2$

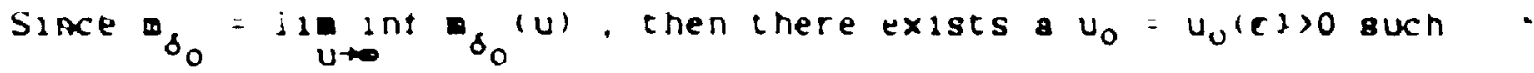
that $a_{\delta_{0}}(u)>a_{0}-\varepsilon / \mathcal{u}$ when $u>u_{0}$. and hence $\delta_{0}(u)>-c$ when uduo. Ihus $\frac{s(t)-s(u)}{u^{r}}>-\varepsilon$ when $u>u_{0}$. urtutuog(u).

Sumarizing, we see that the slowly decreasing condil turl weans that for any $c>0$, there is a $\delta_{0}>0$ and a $u_{0}>0$ such that $\frac{s(t)-s(u)}{u^{r}}>-t$ when $u>u_{0}$, and ut $t\left(u+\delta_{0}(u)\right.$.

The first part of the next lemea is essentialiy due to Hardy [12]. As for the last part this 19 due to Rajagopal [17] who merely gtateg the result. for the sake of completeness we ghall glve a prout.

(2.4) Leme: ( $(12)$, Theorea 239 and $(17)$, Leana 1$)$

(1) If $(u)>0$ and different $1 a b l e$ for $u>1,0(u)+, 0<0^{\prime}(u)(C$, where $C$ is independent of $u$. $\phi(v)^{-}=\int_{1}^{u} \frac{d t}{(t)}$ and, if $\div$

(11) $g(t)=s_{n}, n<t<n+1, \operatorname{set} 1 s+1 e s$

$\lim _{\delta \rightarrow 0+} \lim _{u \rightarrow \lim _{u} t u+\delta b(u)} \frac{g(t)-g(u)}{u^{r}} 20$.

then there are positive constants $K$ and $K^{\prime}$ such that

$s(q)-s(p)>-K q^{r}\left[(q(q)-\phi(p)]-k p^{r}\right.$, for $\left.q>p\right) 1$.

Furtherwore, if

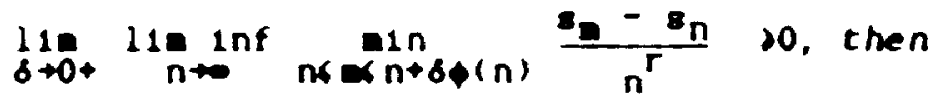


$\left.a_{q}-s_{p}\right)-K q^{r}(\phi(q)-(p))-K^{\prime} p^{r}$, for $q>p>1$.

In particular, taking $(u)=2 \sqrt{u}$,

$\left(T_{r}\right): \lim _{\delta \rightarrow 0+} \lim _{n \rightarrow \infty} \operatorname{lint}_{n \in m+\delta \checkmark n} \frac{s_{n}-s_{n}}{n^{r}}$ so

$\epsilon$

laplies for $q>p>1$.

$$
\begin{aligned}
s_{q}-s_{p} & >-k q^{r}\left(q^{1 / 2}-p^{1 / 2}\right)-K p^{r} \\
& >-K\left(q^{r+1 / 2}-p^{r+1 / 2},-K p^{r} .\right.
\end{aligned}
$$

Proof: By condition (11), there exisis a $\delta_{0}>0$, and a $u_{0}>0$ such

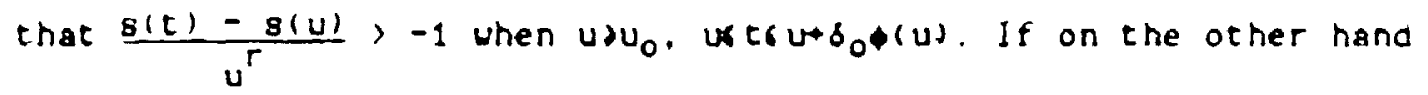
$u u_{0}$, truo $u_{0}+b_{0}\left(u_{0}\right)$, then $\frac{s(t)-s(u)}{u^{r}}$ has a lower bound depenasng only. on $u_{0}$.

Thus there are numbers $r$ and $\delta_{0}$ sich that $\frac{s(t)-g(u)}{u^{r}}>-r$ for all $t$ and $u$ guch that $u t\left(u+\delta_{0} \bullet(u)\right.$.

Now write $p_{0}=p, p_{1}=p_{0}+\delta_{0}\left(p_{0}\right), \ldots, p_{k+1}=p_{k}+\delta_{0}+\left(p_{k}\right) \ldots$ (so that $16 p=p_{0}<p_{1}<p_{2}<\ldots$ and $p_{1} \rightarrow \bullet$ as $1 \rightarrow \bullet$. since

$\left.p_{1}-p_{0}=\sum_{k=0}^{1-1}\left(p_{k+1}-p_{k}\right)=\delta_{0} \sum_{k=0}^{1-1}\left(p_{k}\right)>\delta_{0} \sum_{k=0}^{L-1}\left(p_{0}\right)=\delta_{0}\left(p_{0}\right) 1\right)$

and suppose that $p_{1} 6 q<p_{1+1}$.

For each $\mathrm{j}=0,1, \ldots, 1-1,0<p_{j+1}-p_{j}=\delta_{0}\left(p_{j}\right)$, and

$0<-p_{1}\left(p_{1+1}-p_{1}=\delta_{0}\left(p_{1}\right)\right.$.

Therefore.

$$
\begin{aligned}
g(q)-g(p)= & \sum_{j=0}^{1-1}\left(g\left(p_{j+1}\right)-g\left(p_{j}\right)\right)+s(q)-s\left(p_{1}\right) \\
& \left., \sum_{j=0}^{1-1}\left(-r p_{j}^{r}\right)+\left(-r p_{1}^{r}\right)=-r p^{r}-r \sum_{j=1}^{1} p_{j}^{r}\right)-r p^{r}-r \sum_{j=1}^{1} q^{r}
\end{aligned}
$$


$=-Y p^{r}-r q^{r} 1$

By the wean value theores,

$\phi\left(p_{\jmath+1}\right)-\phi\left(p_{\jmath}\right)=\left(p_{j+1}-p_{j}\right) \phi^{\prime}(\varepsilon)$ for some $\varepsilon \varepsilon\left(p_{j} \cdot p_{j+1}^{2}\right)$. Hence

$\phi\left(p_{j+1}\right)=\phi\left(p_{j}\right)+\left(p_{j+1}-p_{j}\right) \phi^{\prime}(E)=\phi\left(p_{j}\right)+\delta_{0} \phi\left(p_{j}\right) \phi^{\prime}(E)$

$(p),\left(1+\delta_{0} C\right)$, by hypothesis $(1)$

1.e. $\frac{1}{\left(p_{j}\right)}<\frac{1+\delta_{0} C}{\left(p_{j}+1\right.}$

Thus, $1 \delta_{0}=\sum_{j=0}^{1-1} \delta_{0} \frac{\phi\left(p_{j}\right)}{\phi\left(p_{j}\right)}=\sum_{j=0}^{1-1} \frac{p_{j+1}-p_{j}}{\phi\left(p_{j}\right)}\left(11+\delta_{0} C\right) \sum_{j-0}^{1-1} \frac{p_{j}+1-p_{j}}{\phi_{(p j+1}}$. $\left(1+\delta_{0} C\right) \int_{P_{0}}^{P_{1}} \frac{d u}{(u)}\left(11+\delta_{0} C\right) \int_{p}^{q} \frac{d u}{\phi(u)}=\left(1+\delta_{0} C\right)(\phi(u)-\phi(p)]$

Hence, $s(q)-s(p)>-r q^{r} 1-r p^{r}>-r\left(\frac{1}{\delta_{0}}+c\right) q^{r}\left(\phi(q)-\langle(p)]-y p^{r}\right.$. Selting $K-Y\left(\frac{1}{\delta_{0}}+C\right)$, and $K^{\prime}=Y$, which are both positive, we see that the proof of the first part of the lema 18 complete.

As for the latter part of the lema, the hypothesis weans that biven any $c>0$ there is a $\delta_{0}>0$ and an $n_{0}>0$ such that

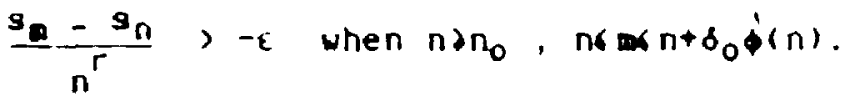

Next, given $t$ and $u, t>u$, let $t<m+1$, nfuxn +1 , so that

$s(t)=s_{0}$, and $s(u)=s_{n}$.

Choose $u_{0}>n_{0}+1$ guch that $\frac{1}{\left(u_{0}-1\right)}<\frac{\delta_{0}}{2}$ and then a positive $\delta^{\circ}<\delta_{0}$ such that $\delta^{\prime}\left(1+\frac{C \delta_{0}}{2}\right)<\frac{s_{0}}{2}$.

For $t \partial u \partial u_{0}$ and $\frac{t-u}{(u)}\left(\delta^{\prime}\right.$ we have that $\left.(t]\right)[u] \partial\left(u_{0}\right) \partial n_{0}$

1.e. asn $n_{0}$, and $\frac{a-n}{(n)}<\frac{t-(u-1)}{(v-1)}=\frac{t-u}{\langle(u-1)}+\frac{1}{(v-1)}$ 
$\left\langle\frac{s^{\prime}(u)}{\phi(v-1)}+\frac{1}{(v-1)}<\delta \cdot\left(1+\frac{c}{\phi(u-1)}\right)+\frac{1}{(y-1)}\right.$, since by the weall

value treores and hypothesis $(1), 0<\phi(u)-(u-1)=\phi^{\prime}(E)<C$ for som

E $(u-1, u)$, and so

$0<\frac{(u)}{(v-1)}-1<\frac{C}{(u-1)}$

Hence $\frac{-n}{(n)}<\delta^{\prime}\left(1+\frac{c}{(u-1)}\right)+\frac{1}{(u-1)}<\delta^{\prime}\left(1+\frac{c}{\phi\left(u_{0}-1\right)}\right)+\frac{1}{\left(1 u_{0}-1\right)}$

$$
<\delta^{\prime}\left(1+\frac{C \delta_{0}}{2}\right)+\frac{\delta_{0}}{2}<\delta_{0} \cdot
$$

Thus we have manzno, $\frac{\Phi=n}{(n)} r \delta_{0}$, and it follows that $\frac{s_{\mathrm{m}}-s_{n}}{n^{r}}>-\varepsilon$.

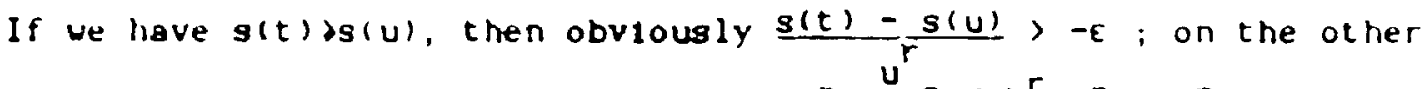
hand if $s(t)<s(u)$, then $\frac{s(u)-\frac{s(t)}{u^{r}}}{r^{2}}=\frac{s_{n} \frac{u^{r}}{s_{m}}}{n^{r}}\left(\frac{n}{u}\right)^{r}, \frac{s_{n}-\frac{s}{m}}{n^{r}}<\varepsilon$.

Thus, $\frac{s(t)-s(u)}{u^{r}},-\varepsilon$, when $t \geqslant u>u_{0}, \frac{t-u}{\varphi(u)} \delta^{\circ}$.

Finally, following the proof of the first part of this leama we have: $s(q)-s(p)>-K q^{r}[\phi(q)-\phi(p)]-K^{\prime} p^{r}$, for $\left.q>p\right) 1$, and where $K, K^{\prime}$ are positive constants. Specializing to integers $p$ and $q$, so that

$g(p)=g_{p}$ and $g(q)=s_{q}$ we heve os required:

$a_{q}-a_{p}>-K_{q}^{r}[(q)-(p)]-K^{\prime} p^{r}$, for $q \partial p 21$.

When $(u)=2 J$, then $(u)=J_{u}-1$, and so $\left(T_{r}\right)$ inplies for $q \partial p \lambda 1$, $s_{q}-s_{p}>-K q^{r}\left(q^{1 / 2}-p^{1 / 2}\right)-K p^{r}$, and hence

$$
\begin{aligned}
a_{q}-g_{p}>-K q^{r+1 / 2}+K q^{r} p^{1 / 2}-K p^{r} & >-K q^{r+1 / 2}+K p^{r+1 / 2}-K \cdot p^{r} \\
& =-K\left(q^{r+1 / 2}-p^{r+1 / 2}\right)-K \cdot p^{r}
\end{aligned}
$$

where $K, K$ ere positive conatants. //I 
(2.5) Reaprk: In particular it $a_{n}:=\omega_{n}-s_{n-1}=q_{1} n^{r-1 / 2},, r 20$ (s.e. $s_{n},-k n^{r-1 / 2}$ for sose $k$ so, and $n=1,2, \ldots$, then $\left(T_{r}\right)$ nolds.

To see this consider the following:

$\frac{s_{m}-s_{n}}{n^{r}}=\frac{1}{n^{r}} \sum_{j=n+1}^{e} a_{j},-\frac{k}{n^{r}} \sum_{j=n+1}^{k} j^{r-1 / 2},-\frac{k(n-n)}{d(n+1)}\left(\frac{a}{n}\right)^{r}$

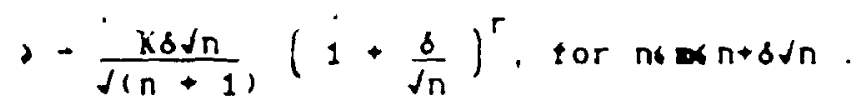
$2=\operatorname{ks}\left(1+\frac{\delta}{\sqrt{n}}\right)^{r}$.

and $\underset{n \leqslant m i n+\delta \sqrt{n}}{\min } \frac{s_{m}-s_{n}}{n^{r}},-k \delta\left(1+\frac{\delta}{d_{n}}\right)^{r}$.

Thus.

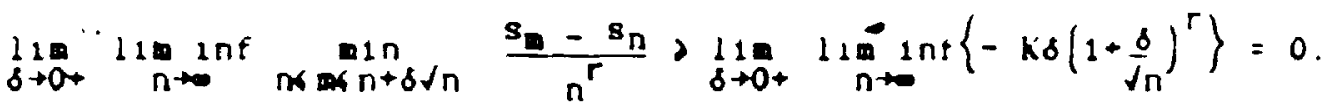

(2.6) Lem: Let $M$ and $N$ be any posstive integers such that $n>x / a+t \delta(x / a)$. No<N<x/a-tJ(x/a). Then for any $r>0$, and $x, t \rightarrow-$ we have:

(2.7) $\sum_{n=N_{0}}^{N} n^{r} c_{n}(x)=o\left(x^{r}\right)$.

(2.8) $\sum_{n=n}^{\infty} n^{r} c_{n}(x)=o\left(x^{r}\right)$.

(2.9) $\sum_{n=N}^{H} n^{r} c_{n}(x)-(x / \alpha)^{r}$. 
(2.10) $\sum_{n=n}^{\infty}\left(n^{r+1 / 2}-n^{r+1 / 2}\right) c_{n}(x)=o\left(x^{r}\right)$.

(For example definition (2.9) means, as the others siallarly

do, the following: for every $\varepsilon>0$ there exists an $x_{0}$

such that for every $x>x_{0}, t>x_{0}$, and for every $K$ and $N$,

sat 1 sfy 1 ing $H>x / \alpha+t J(x / a), N_{0}<N<x / a-t J(x / a)$ we have

$\left.\left|\frac{1}{x^{r}} \sum_{n=N}^{n} n^{r} c_{n}(x)-\frac{1}{a^{r}}\right|<\varepsilon.\right)$ Proof: Regarding $(2.7)$ we first show that $\left.\sum_{n=N_{O}}^{N} c_{n}(x)=011\right)$
as $x, t \rightarrow \infty$, and fron this we deduce the final result.

$$
\begin{aligned}
\sum_{n=N_{0}}^{N} c_{n}(x) & \sum_{N_{0} \delta n<x / a-t f(x / a)} c_{n}(x) \\
& =\left(\sum_{N_{0} 6 n<x / a-x^{5}}+\sum_{x / a-x^{5}(n<x / a-t /(x / a)}\right) c_{n}(x)=s_{1}+s_{2},
\end{aligned}
$$

where $1 / 2<E<2 / 3$.

By Lema (2.3) with $r=0, \$_{+1}=o(1)$ as $x \rightarrow \infty$.

(S1gfe $3 F-2<0$, Leand $\left(2 \frac{2}{2}\right)$ inplies that

$c_{n}(x)=0\left\{x^{-1 / 2} \exp \left(-\frac{a^{2} k_{n}^{2}}{2 x}\right)\right\}$ for $\left|k_{n}\right|=|x / a-n| 6 x^{5}$. In particular this est laste is valid for $n$ such that $\left.x^{E} \partial x / \alpha-n\right) t f(x / \alpha)$.

Thus,

$$
\begin{aligned}
s_{2} & \left.=\alpha x_{x / a-x^{E}} \sum_{n<x / a-t /(x / a)} \exp \left(-\frac{a^{2} k_{n}^{2}}{2 x}\right)\right\} \\
& \left.=\alpha x^{-1 / 2} \sum_{n=n_{0}}^{n_{1}} \exp \left(-\frac{a^{2} k_{n}^{2}}{2 x}\right)\right\}, \text { where } n_{0} \text { 1s the sallest }
\end{aligned}
$$


integer $3 x / a-x^{E}$, and $n_{1} 28$ che largest integer $<x / a-t h(x / a)$. Let $f(y)=\exp \left(-\frac{a^{2} y^{2}}{2 x}\right)$. Then $f(y)$ decreases for all $y>0$. For $x$ and $t$ non-negat ive and by our cholce of $n_{1}$ the inequality: $0, t d(x / a)<x / a-n_{1}=k_{n_{1}}$ is valid. Applying Leman (1.7) we have:

$$
\begin{aligned}
\sum_{n=n_{0}}^{n_{1}} \exp \left(-\frac{a^{2} k_{n}^{2}}{2 x}\right) & \exp \left(-\frac{a^{2} k_{n_{1}}^{2}}{2 x}\right)+\int_{k_{n_{1}}}^{k_{n_{0}}} \exp \left(-\frac{a^{2} y^{2}}{2 x}\right) d y \\
& \cdot \exp \left\{-\frac{\alpha^{2}}{2 x}(t J(x / \alpha))^{2}\right\}+\int_{t \sqrt{ }(x / \alpha)}^{x^{\varepsilon}} \exp \left(-\frac{a^{2} y^{2}}{2 x}\right) d y
\end{aligned}
$$

since $x^{E} \partial x / a-n_{0}=k_{n_{0}}$ and $t d(x / a)<x / a-n_{1}=k_{n_{i}}$.

It follows that $\sum_{n=n_{0}}^{n_{1}} \exp \left(-\frac{\alpha^{2} k_{n}^{2}}{2 x}\right) \cdot \exp \left(-\frac{a t^{2}}{2}\right)+\int_{t J(x / \alpha)}^{\infty} \exp \left(-\frac{\alpha^{2} y^{2}}{2 x}\right) d y$

$$
=\exp \left(-\frac{a t^{2}}{2}\right)+\frac{d(2 x)}{a} \int_{J(\alpha / 2) t}^{\infty} \exp \left(-u^{2}\right) d u \text {. }
$$

Thus, $s_{2}=o\left\{x^{-1 / 2} \exp \left(-\frac{\alpha t^{2}}{2}\right)\right\}+\alpha\left\{\int_{J(\alpha / 2) t}^{\infty} \exp \left(-u^{2}\right) d u\right\}=o(1)$ as $x, t \rightarrow \infty$.

Finally, $\sum_{n=N_{0}}^{N} c_{n}(x)+S_{1}+S_{2}=o(1)+o(1)=o(1)$ as $x, t \rightarrow \cdots$, and hence $\sum_{n=N_{0}}^{N} n^{r} c_{n}(x), \mathbb{N}^{r} \sum_{n=N_{0}}^{N} c_{n}(x)<(x / a)^{r} o(1)=o\left(x^{r}\right)$ as $x, t \rightarrow \cdots$ for $(2.8)$ we nust show that $S:=x^{-r} \sum_{n=n}^{\infty} n^{r} c_{n}(x)=0(1)$ as $x, t \rightarrow \infty$. $S=x^{-r}\left(\sum_{k<n \times / a+x^{\xi}}+\sum_{n>x / a+x^{E}}\right)^{r} c_{n}(x)=s_{1}+s_{i} \cdot 1 / 2<E<2 / 3$. By Leme (2.3), $\mathrm{S}_{2}=0(1)$ as $\times \rightarrow \bullet$ 
Mexe,

$$
\begin{aligned}
& s_{1}=\left\{x^{-r} e^{-x} \sum_{x / \alpha+c \sqrt{ }(x / \alpha)<n x / \alpha+x^{5}} n^{r} \frac{x^{\alpha n+\beta-1}}{\Gamma \operatorname{con}+\beta)}\right\} \text {. since } n>x / \alpha+\varepsilon \sqrt{ }(x / a) \\
& =\left\{e^{-x} \sum_{x / \alpha+c \delta(x / \alpha)<n 6 x / \alpha+x^{E}} \frac{x^{\alpha n+\beta-r-1}}{\Gamma(\alpha+\beta-r)}\right\} \text {. Dy Leans } 12.21
\end{aligned}
$$

Since $35-2<0$, Lewa $(2.1)$ inplies that

$\frac{e^{-x} x^{\alpha n+\beta-r-1}}{r(\alpha n+\beta-r)}=\left\{x^{-1 / 2} \exp \left(-\frac{\alpha^{2} n_{n}^{2}}{2 x}\right)\right\}$ for $\left|n_{n}\right|=|n-x / \alpha| 6 x^{6}$. In

perticular this eacieate us vaild far o such that $t /(x / a)<n-x / a r x^{E}$.

Therefore, $s_{1}=\left\{x^{-1 / 2} \sum_{x / \alpha+t J(x / \alpha)<n<x / \alpha+x^{\varepsilon}} \exp \left(-\frac{\alpha^{2} n_{n}^{2}}{2 x}\right)\right\}$

$$
=\left\{x^{-1 / 2} \sum_{n=n_{0}}^{n_{1}} \exp \left(-\frac{a^{2} n_{n}^{2}}{2 x}\right)\right\} \text {, where } n_{0} \text { 1s the saillest }
$$

Lateger $2 x / a+t J(x / a)$, and $n_{1}$ 1s the largest integers $x / a+x^{E}$.

Applying Lema (1.6) with $f(y)=\exp \left(-\frac{a^{2} y^{2}}{2 x}\right)$ we $f$ ind, as in the proof of (2.7), that $s_{1}=o(1)$ as $x, t \rightarrow \bullet$.

Thue, 0 \& $S_{1}+S_{2}=o(1)+o(1)=o(1)$ as $x, t \rightarrow \infty$.

(2.9) for $r=0$ ? obviously followe from (2.7) and (2.8) with $r=0$, and frov the fact that $\sum_{n=N_{0}}^{\infty} c_{n}(x) \sim 1$ as $x \rightarrow-$. Before we prove the result for $r>0$ we ank the following coments which will aid us in the proof.

Firat, (2.7) any be written in the following equivalent fors: 
for any sequences $\left\{N_{1}\right\},\left\{t_{1}\right\},\left\{x_{1}\right\}$ such that $t_{1} \rightarrow-, x_{1} \rightarrow \bullet$. and $N_{0}\left(N_{1}<x_{1} / a-t_{1} f\left(x_{1} / \alpha\right)\right.$ then $\sum_{n=N_{0}}^{N_{1}} n^{r} c_{n}\left(x_{1}\right)=0\left(x_{1}{ }^{r}\right)$ as $1 \rightarrow \cdots$

Secondly, (2.8) way written in the following equivalent for: : for any sequences $\left\{H_{1}\right\},\left\{t_{1}\right\},\left\{x_{1}\right\}$ such that $t_{1} \rightarrow \infty, x_{1} \rightarrow \infty$; and $\left.H_{1}\right) x_{1} / a+t_{1} f\left(x_{1} / a\right)$ then $\sum_{n=H_{1}}^{\infty} n^{r} c_{n}\left(x_{1}\right)=o\left(x_{1}^{r}\right.$, as $1 \rightarrow \infty$. Lastly. (2.9) ay be uritten in the following equivalent for : for any sequences. $\left\{M_{1}\right\},\left\{N_{1}\right\},\left\{t_{1}\right\},\left\{x_{1}\right\}$ such that $t_{1} \rightarrow \bullet$, $x_{1} \cdots \infty$, and $n_{1}>x_{1} / \alpha+t_{2} v\left(x_{1} / \alpha\right), N_{0}<N_{1}<x_{1} / \alpha-t, d\left(x_{1} / \alpha\right)$ then $\sum_{n=N_{1}}^{n_{Y}} n^{r} c_{n}\left(x_{1}\right) \ldots\left(x_{1} / a\right)^{r}$ as $1 \rightarrow \infty$.

Nou to prove (2.9) let us choose arbitrary sequences $\left.\left\{M_{1}\right\},\left\{N_{1}\right\},\left\{t_{1}\right\},\left\{x_{1}\right), t_{1} \rightarrow \infty, x_{1} \rightarrow \infty, n_{1}\right) x_{1} / a+t_{1} J\left(x_{1} / a\right)$ and $N_{0}<N_{1}<x_{1} / \alpha-t_{1} f\left(x_{1} / a\right)$.

Having established (2.7) and (2.8) we will use the 1 r equivalent forms and prove that $\sum_{n=N_{1}}^{K_{1}} n^{r} c_{n}\left(x_{1}\right) \sim\left(x_{1} / \alpha\right)^{r}$ as $1 \rightarrow \infty$.

Now having already chosen $\left[t_{1}\right],\left[x_{1}\right], t_{1} \rightarrow-, x_{1} \rightarrow-$, choose another sequence $\left[w_{1}\right]$ such that $0<v_{1} 6 t_{1}, w_{1} \rightarrow$ and $w_{1} / f\left(x_{1} / a\right) \therefore 0$, $281 \rightarrow \cdots$.

This is possible by letting $w_{1}=\operatorname{ain}\left\{\left(x_{1} / a\right)^{1 / 4}, c_{1}\right\}$.

Having already chosen $\left(H_{1}\right)$ and $\left[H_{1}\right\}$, choose sequences of positive integers $\left[H_{1}^{\prime}\right\}$ and $\left[N_{1}^{\prime}\right]$ such that

$$
H_{1}-16 x_{1} / \alpha+w_{1} /\left(x_{1} / \alpha\right)<H_{1} / H_{1}
$$


$\operatorname{ard} H_{1} \ll N_{1}<x_{1} / a-w_{1} J\left(x_{1} / a\right)\left(N_{1}+1\right.$.

Then,

(2.11)

$$
\sum_{n=N_{1}}^{N_{1}} n^{r} c_{n}\left(x_{2}\right)=\left\{\sum_{n=N_{1}}^{N_{1}^{\prime}-1}+\sum_{n=N_{1}^{\prime}}^{n_{1}^{\prime}}+\sum_{n=N_{1}^{\prime}+1}^{N_{1}}\right\} n^{r} c_{n}\left(x_{1}\right)
$$

(Thesfirst series on the right side of 12.11 ) is defined to be zero when $N_{1}=N_{1}$, as 18 the last serles when $M_{1}^{\prime}=H_{1}$.)

Since $x_{1} \rightarrow \infty$, and ${v_{1}}_{1} / f\left(x_{1} / a\right) \rightarrow 0$ as $1 \rightarrow \infty$, we have: $\left(x_{1}\right)^{r} \sim\left(x_{1} / \alpha\right)^{r}$ and $\left(n_{1}\right)^{r} \sim\left(x_{1} / \alpha\right)^{r}$ as $1 \rightarrow \infty$

Hence frow the inequality:

$\left(N_{1}\right)^{r} \sum_{n=N_{1}^{\prime}}^{M_{1}^{\prime}} c_{n}\left(x_{1}\right), \sum_{n=N_{1}}^{M_{1}^{\prime}} n^{r} c_{n}\left(x_{1}\right),\left(n_{1}^{\prime}\right)^{r} \sum_{n=N_{1}^{\prime}}^{n_{1}^{\prime}} c_{n}\left(x_{1}\right)$

1t follows that $\sum_{n=N_{1}^{1}}^{n} n^{r} c_{n}\left(x_{1}\right)-\left(x_{1} / a\right)^{r} \sum_{n=N_{1}^{\prime}}^{n} c_{n}\left(x_{1}\right)$

$=\left(x_{1} / \alpha\right) r\left\{\sum_{n=N_{0}}^{\infty}-\sum_{n=N_{0}}^{N_{1}^{\prime}-1}-\sum_{n=n_{1}^{\prime}+1}^{\infty}\right\} c_{n}\left(x_{1}\right)$ as $1 \rightarrow \cdots$

Since $w_{1} \rightarrow \bullet, x_{1} \rightarrow-$ as $1 \rightarrow \infty$, and $H_{1}^{\prime}, n_{1}^{\prime}$ are respectively less than $x_{1} / a-w_{1} J\left(x_{1} / \alpha\right)$ and greater than $x_{1} / \alpha+w_{1} f\left(x_{1} / \alpha\right)$, the results. (2.7) and (2.8) in their equivalent forme nold, es observed at the outget of this proof.

Therefore, 


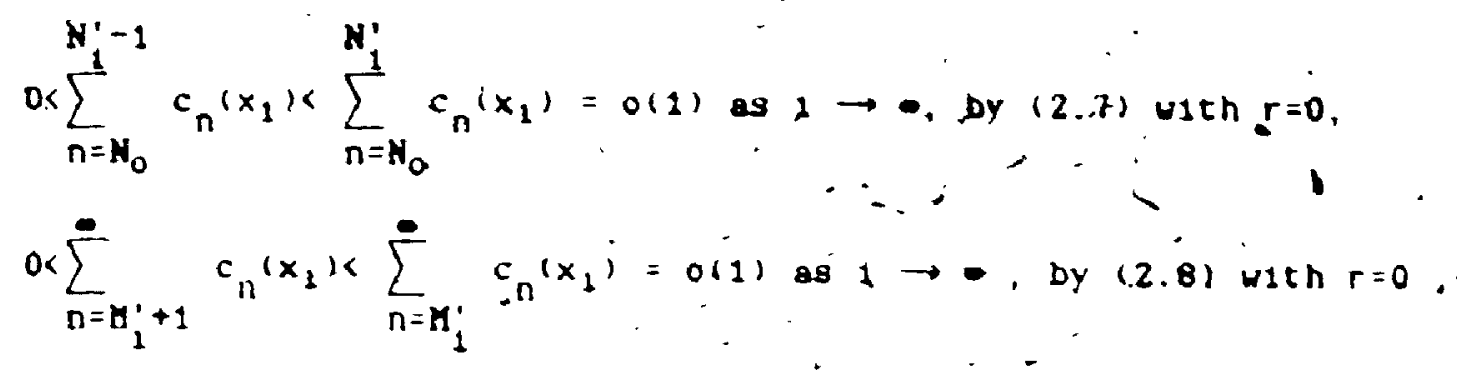
and moreover $\sum_{n=N_{0}}^{\infty} c_{n}\left(x_{1}\right)-1$ as $1 \rightarrow \infty$ since $\sum_{n=N_{0}}^{\infty} c_{n}(x)-1$.

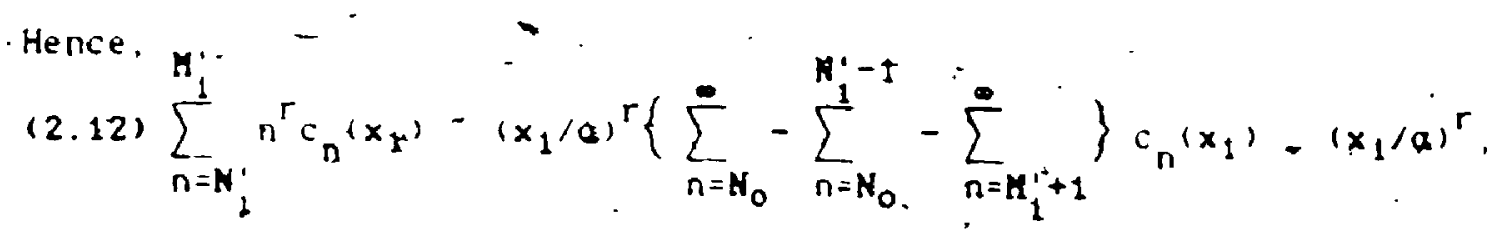
as $1 \rightarrow \infty$.

(2. $\beta) \quad \operatorname{ar} \sum_{n=N_{1}}^{N-1} n^{r} c_{n}\left(x_{1}\right)<\sum_{n=N_{0}}^{N} n^{r} c_{n}\left(x_{1}\right)=o\left(x_{1}^{5} ;\right.$ as $1 \rightarrow \cdots$, by $(2.7)$.

(2.14) or $\sum_{n=n_{1}+1}^{n_{1}} n^{r} c_{n}\left(x_{1}\right)<\sum_{n=n_{1}^{\prime}}^{\infty} n^{r} c_{n}\left(x_{1}\right)=o\left(x_{1}^{r}, \text { as } 1 \rightarrow \infty \text {. by } 12.8\right)^{\circ}$

Substituting (2.13), (2.12), and $(2.14)$ in $(2.11)$ gives

$\sum_{n=N_{1}}^{n_{1}} n^{r} c_{n}\left(x_{1}\right) \sim\left(x_{1} / a\right)^{r}$ as $1 \rightarrow \cdots$

The final result now follow from the gquivalence mentioned at the Deginning of this proof.

For 12.10$)$, we aust show that.

$S:=\sum_{n=u^{\prime}}^{\infty}\left(n^{r+1 / 2}-n^{r+1 / 2}\right) c_{n}(x)=o\left(x^{r}\right) \omega x, t \rightarrow \cdots$.

By the mean value theorea it follow that 
$n^{r+1 / 2}-n^{r+1 / 2},(2 r+1) n^{r}\left(n^{1 / 2}-n^{1 / 2}\right)$ and hence

orS $(2 r+1) \sum_{n=n}^{\infty}\left(n^{1 / 2}-n^{1 / 2}, n^{r} c_{n}(x)\right.$. Therefore $1 t$ sufilces to prove the following

clese: $s_{1}:=x^{-r} \sum_{n=n}^{\infty}\left(n^{1 / t}-n^{1 / 2}, n^{r} c_{n}(x)=0(1)\right.$ as $x, t \rightarrow \infty$.

Since $n>x / a$ at toliows that $n^{1 / 2}+n^{1 / 2}, 2 n^{1 / 2}, 2 J(x / a)$ ano

$(n-n)=\left(n^{1 / 2}-n^{1 / 2}:\left(n^{1 / 2}+n^{1 / 2}, \geqslant 2 f(x / a)\left(n^{1 / 2}-n^{1 / 2}\right)\right.\right.$.

Thup,

$$
\begin{aligned}
& s_{1} \cdot \frac{a^{1 / 2} x^{-r-1 / 2}}{2} \cdot \sum_{n=n}^{\infty}(n-n) n^{r} c_{n}(x) \\
& =\frac{a^{1 / 2} x^{-r-1 / 2}}{2}\left\{\sum_{n<n<x / a+x^{E}} \cdot \sum_{n>x / a+x} s_{1, n}\right\}(n-n) n^{r} c_{n}(x) \\
& -s_{1,1}+s_{1,2} \text {, where } 1 / 2<E<2 / 3 .
\end{aligned}
$$

Now,

$$
\begin{aligned}
& S_{1,1}=\left\{x^{-r-1 / 2} e^{-x} \sum_{n\left(n \alpha x / \alpha+x^{E}\right.}(n-n) n^{r} \frac{x^{\alpha(r+\beta-1}}{I(\alpha n+\beta)}\right\}
\end{aligned}
$$

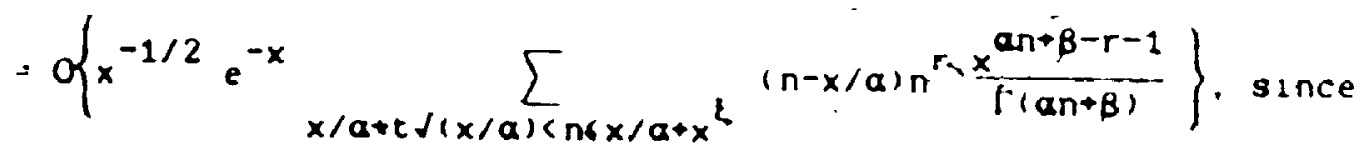

$n>x / \alpha+t d(x / \alpha) a x / a$, and $n-n<n-x / \alpha$.

By Leme $(2.2)$,

$s_{1,1}-\left\{x^{-1 / 2} e^{-x} \sum_{x / \alpha+t \delta(x / \alpha)<n<x / \alpha+x}(n-x / \alpha) \frac{x^{\alpha+\beta+\beta-1}}{r(\alpha+\beta+\gamma)}\right\}$.

As $35-2<0$, Leme 12.1 ) 1aplies that

$\frac{e^{-x} x^{\alpha n+\beta-r-1}}{\Gamma(a n+\beta-r)}=U\left\{x^{-1 / 2} \exp \left(-\frac{a^{2} h_{n}^{2}}{2 x}\right)\right\}$ for $\left|h_{n}\right|=|n-x / \alpha| 6 x^{6}$. 
It follows that tor $n$ guch that $t /(x / a)<n-x / a r x^{5}$

$e^{-x} \frac{x^{a n+\beta-r-1}}{\Gamma(a n+\beta-r)} n_{n}=u\left\{x^{-1 / 2} n_{n} \exp \left(-\frac{a^{2} n_{n}^{2}}{2 x}\right)\right\}$

Therefore, $\left.s_{1,1}=u_{x}^{-1} \sum_{x / a+t \gamma(x) a)+n+x / a+x^{2}} n_{n} \exp \left(-\frac{a^{2} n_{n}^{2}}{2 x}\right)\right\}$ $u\left\{x^{-1} \sum_{\substack{n=n_{0} \\ n_{1}}} n_{n} \exp \left(-\frac{a^{2} n_{n}^{2}}{2 x}\right)\right\}$. where no is the

smallest integer; $x i a+t /(x / a)$. and $n_{1}$ is the largest linceger $6 x / a+x^{2}$

Let $f(y)-y \exp \left(-\frac{a^{2} y^{2}}{2 x}\right)$. Then $f(y)$ decreases for all $y \partial \sqrt{x / a}$. For $t \geqslant 1 / \sqrt{a}$, and by our cholce of no the inequalicy Jx/aref(x/a)<no $-x / a=n_{n_{0}}$ ls vìlid. By Lema $(1.6)$.

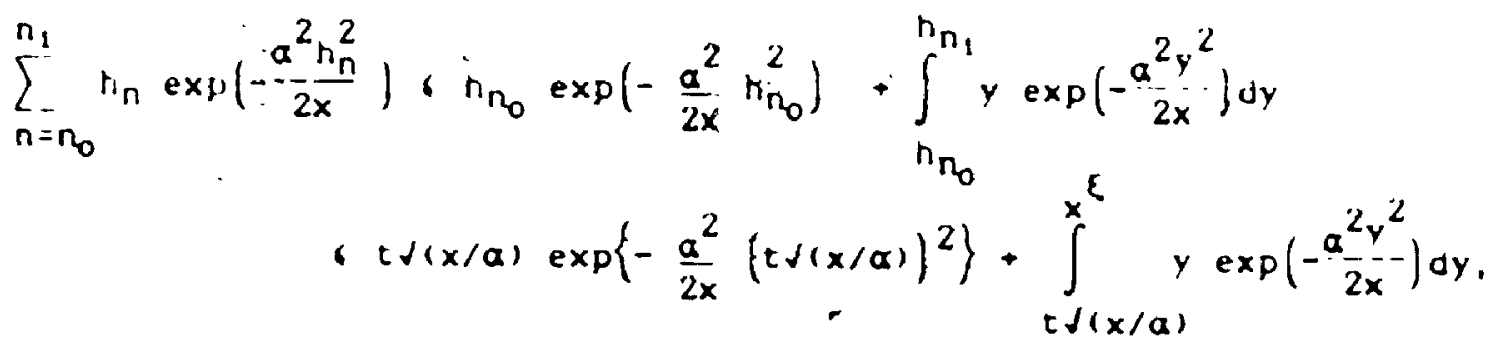

since $x^{\xi} \partial n_{1}-x / \alpha-n_{n_{t}}$ and $t J(x / \alpha)<n_{0}-x / a-n_{n_{0}}$.

Further,

$$
\sum_{n=n_{0}}^{n_{1}} n_{n} \exp \left(-\frac{a^{2} n_{n}^{2}}{2 x}\right) \cdot t J(x \cdot a) \exp \left(-\frac{a^{2}}{2}\right)+\int_{t J(x / a)}^{\infty} y \exp \left(-\frac{a^{2} y^{2}}{2 x}\right) d y
$$

Therefore.

$$
S_{1,1}=0\left\{x^{-1 / 2} t \exp \left(-\frac{a t^{2}}{2}\right)\right\}+\alpha\left\{x^{-1} \int_{t f(x / a)}^{0} y \exp \left(-\frac{a^{2} y^{2}}{2 x}\right) d y\right\}
$$


finally,

$$
=o(1)+\left\{\int_{J(a / 2) t}^{0} u \exp \left(-u^{2}\right) d u\right\}=o(1) \text { as } x, t \rightarrow \cdots
$$

$s_{1,2}=\left\{x^{1 / 2} e^{-x} \sum_{n>x / a+x^{5}} n^{r+1} \frac{x^{a n+\beta-r-2}}{r(a n+B)}\right\}$

$=\left\{x^{1 / 2} e^{-x} \sum_{n>x / a+x^{5}} \frac{x^{a n+\beta-r-2}}{\Gamma(a n+\beta-r-1)}\right\}$, br Lema (2.2)

$=0\left(x^{1 / 2} e^{-x^{\eta}}\right)$, $0<\eta<2 \varepsilon-1$, by Lemo $(2.1)$

$=0(1)$ as $\times \rightarrow$.

Thus, $0 \times S_{1}\left(S_{1,1}+S_{1,2}=o(1)+o(1)=0(1)\right.$ as $x, t \rightarrow \cdots$.

This proves the claia, and as explarned at the outset the proof of

(2.10) 13 now complete. /1/

(2.15) Theorea: If $b(x)=O\left(x^{r}\right)$ as $x \rightarrow \infty$ and $\left(T_{r}\right)$ holds, then $s_{n}=O\left(n^{r}\right)$.

Proof: Following Sitaraman ([18], proof of Theored 1) urite,

$\sigma_{n}=\frac{s_{n}}{n}(n \geqslant 1), \quad \sigma_{1}(n)=\max _{v(n} \sigma_{v}, \sigma_{2}(n)=\max _{v 6 n}\left(-\sigma_{v}\right)$.

There are two $\log 1 \mathrm{cal}$ possibilities:

Cafe $(A): \sigma_{1}(n) 2 \sigma_{2}(n)$ for infinitely wany values of $n$.

or Caee (B): $\sigma_{1}(n)\left\langle\sigma_{2}(n)\right.$ for all n sufficlently large.

We prove thot the assunptions case $(A),\left(\sigma_{n}\right)$ unbounded. and $\left(T_{r}\right)$ lead to.

a contradiction of the hypothesis $b(x)=O\left(x^{r}\right)$ as $x \rightarrow-$, and also that

case $(B),\left(\sigma_{n}\right)$ unbounded, and $\left(T_{r}\right)$ contradict the hipothesis $b(x)=O\left(x^{r}\right)$

is $x \rightarrow \bullet$.

Suppose first that case (A) holds and that $\left[\sigma_{n}\right] 18$ unbounded.

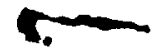


We first show that $\sigma_{:}(n) \rightarrow$ as $n \rightarrow \cdots$

If $\left(\sigma_{1}(n) Y_{1}\right.$ is bounded above, then there exists an $L$ such that

$\sigma_{1}(n)+i$ for ail $n$. This in turn deplies that ont for all n. On the other hand, because of case $(A)$, we have $\sigma_{2}(n) b_{1}$ for ant 1 initely eany

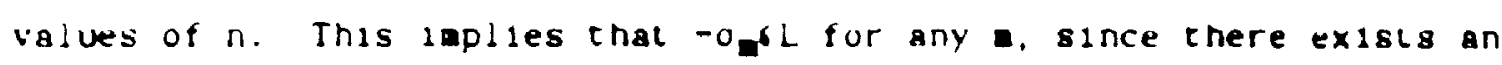
$n$ such that $-0_{0}\left(\sigma_{2}+n\right) 6 L$ for any ann. lience altogether we have shown that lonith for all $n$, which is a contradiction. Thus $\left[\sigma_{1}(n)\right]$ is unbounded above, oureover lt 13 increasing in $n$, nence $o_{i}(n) \rightarrow-$ as

Nov write,

-5.

(2.16) $D(x)=\left(\sum_{n=N_{0}}^{N-1}+\sum_{n=N}^{n-1}+\sum_{n=n}^{\infty} f_{n}(x) g_{i}^{*}=T_{1}(x)+\tau_{2}(x)+T_{3}(x)\right.$

where flrst $N$ and then $M$ are chosen as foljows. Corresponding to any positive $H) H_{0}=\sigma_{1}\left(N_{0}\right)$ there are $N=N(H)>N_{0}$ tor which

(2.17) $\sigma_{N}=\sigma_{1}(N)>2 H, \sigma_{1}(N)>\sigma_{2}(N)$

We choose the least $N=N(H)$ sat 18 fying $(2.17)$.

(To see that there are $N=N(H) N_{0}$ auch that $(2.1 \%) 18$ sat 13 f 1 ed consider the following: the fact that $\sigma_{1}(n) \rightarrow-$ as $n \rightarrow-$, and the hypothesis of case (A) 1 mply that for any positive $H>H_{0}$ there exiata an $N^{\prime}$ such that $\sigma_{1}\left(N^{\prime}\right)>2 H$ and $\sigma_{1}\left(N^{\prime}\right) 2 \sigma_{2}\left(N^{\prime}\right)$. Clearly $\sigma_{1}\left(N^{\prime}\right)=\sigma_{N}$ for some $N^{\prime} N^{\prime}$, and hence $\sigma_{1}\left(N^{\prime}\right) 2 \sigma_{1}\left(N^{\prime}\right) \sigma_{N}=\sigma_{1}\left(N^{\prime}\right)$. Thus $\sigma_{N}=\sigma_{1}\left(N^{\prime}\right)=\sigma_{1}\left(N^{\prime}\right)$ and it follows that $\sigma_{N}=\sigma_{1}\left(N^{\prime}\right) 2 H$. Furthereore, $\sigma_{2}\left(N^{\prime}\right) 2 \sigma_{2}\left(N^{\prime}\right.$ iapllae that $\sigma_{1}\left(N^{\prime}\right)=\sigma_{1}\left(N^{\prime}\right) 2 \sigma_{2}\left(N^{\prime}\right) 2 \sigma_{2}\left(N^{\prime}\right.$. Lagt ly $\left.N\right) N_{0}$, since $2 H>H_{0}=\sigma_{1}\left(H_{0}\right)$ 1apl 1es that $\sigma_{1}(N)>2 H>\sigma_{1}\left(N_{0}\right)$

Next choose the least $M-M(H)>K$ for which

(2.18) $\sigma_{n} \leqslant \frac{1}{2} \sigma_{N}=\frac{1}{2} \sigma_{1}(N)$ 
fThere are such $n$ when $H$ is large. because of the following argunents.

First we prove the

Cla1e: There exists an $H_{1}$ 2Ho such that $\sigma_{n} \times H_{1}$ for infinitelv many

Falues of $n$. In order to obtain a contradiction suppose the clail is false. Therefore, for all $H_{1}>H_{0} . \sigma_{n}>H_{1}$ for all $n$ sufflcientlv large, 1.e. $\sigma_{n} \rightarrow$ as $n \rightarrow \infty$. Now since there can onlv be finitelv manv values of $n$ such that $\sigma_{n}<0$, and since the choice of $N_{0}$ is idaterial

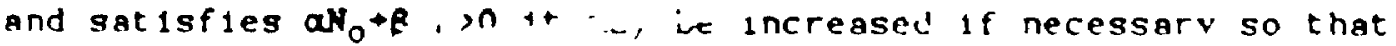
$\sigma_{n}>0$ for $n>N_{0}$, and $\alpha_{0}+\beta-r>0$.

By the proof of Leman (2.2), $\frac{(a n)^{r} \Gamma(a n+\beta-r)}{\Gamma(\alpha n+\beta)}-1$ as $n \rightarrow \infty$. There is therefore a positive constant $L$ such that $\frac{(a n)^{\Gamma} \Gamma(a n+\beta-r)}{\Gamma(a n+\beta)}, L, n d N_{0}$. Hence $\frac{n^{r} \sigma_{n}}{\Gamma(a n+B)}+C \frac{\sigma_{n}}{\Gamma(a n+B-r)}, n \partial H_{0}$. Where $C=\frac{L}{a^{r}}$ is a positive constant.

Thus, $b(x)=\sum_{n=N_{0}}^{\infty} c_{n}(x) s_{n}=a e^{-x} \sum_{n=N_{0}}^{\infty} \sigma_{n} n^{r} \frac{x^{a n+\beta-1}}{\Gamma(a n+B)}$

Hence.

$$
\operatorname{Cade}^{-x} \sum_{n=N_{0}}^{\infty} \sigma_{n} \frac{x^{a n+\beta-1}}{\Gamma(a n+\beta-r)} .
$$

(2.19) $\frac{b(x)}{x^{r}}+\operatorname{Cae}^{-x} \sum_{n=N_{0}}^{-} o_{n} \frac{x^{a n+\beta-r-1}}{r(a n+\beta-r)}$

Now since the $(B, \alpha, \beta)$ nethod 18 regular in the usual sense, and a positive method. It 18 ' totally regular method (see Hardy [12], Theorem 9). Therefore $\sigma_{n} \rightarrow \bullet$ as $n \rightarrow-$, Implies that $\sigma_{n} \rightarrow-(B, \alpha, B-r)$, and hence from $(2.19) \frac{b(x)}{x^{r}} \rightarrow \bullet$, which is a contradiction uith the irpothesis $b(x)=O\left(x^{r}\right)$ as $x \rightarrow \bullet$. Having proven the clala, we have for all $\mathrm{H}_{2 H_{1}}, \sigma_{n}{ }^{H} H$ for infinitely 
aany values of $n$. Bv $(2.17)$ we have $\sigma_{n}<H<\frac{1}{2} \sigma_{N}=\frac{1}{2} \sigma_{1}(N)$ for infinitely anv values of $n$. Thus when $H$ is large there are $M=M(H): N$ sat sfying $\sigma_{M}, \frac{1}{2} \sigma_{N}=\frac{1}{2} \sigma_{1}(N)$.)

Since $\left(T_{r}\right.$ ) holds. Lema (2.4) laplies for $\left.M, N\right) 1$. $s_{K}=s_{N}>-K M^{r}\left(M^{1 / 2}-N^{1 / 2}\right)-K \cdot N^{r}$, where $K$ and $K$, are some positive constants. The cholce of $M$ and $N$ A $(2.18)$ and $(2.17)$ are such that $s_{M}-s_{N}=\sigma_{M} M^{r}-\sigma_{N} N^{r} \cdot \frac{1}{2} \sigma_{N} H^{r}-\sigma_{N} H^{r}-\sigma_{1}(N)\left(\frac{1}{2} M^{r}-N^{r}\right)$.

Therefore, $\sigma_{1}(N)\left(\frac{1}{2} H^{r}-N^{r}\right)>s_{M}-s_{N}>-K M^{r}\left(M^{1 / 2}-N^{1 / 2}\right)-K^{r} H^{r}$.

Since $M>N$ it follows that.

$K\left(H^{1 / 2}-N^{1 / 2}\right)>\sigma_{1}(N)\left\{\left(\frac{N}{n}\right)^{r}-\frac{1^{*}}{2}\right\}-K^{\prime}$.

Now for a flxed \& $6(0,1 / 2)$ we have elther $\left(\frac{N}{n}\right)^{r}>\frac{1}{2}+\delta$ or $\left(\frac{n}{N}\right)^{r},\left(\frac{1}{2}+\delta\right)^{-1}>1$.

In the first case. $K\left(M^{1 / 2}-N^{1 / 2}\right)>\sigma_{1}(N)\left\{\left(\frac{N}{M}\right)^{r}-\frac{1}{2}\right\}-K^{\prime}>\delta \sigma_{1}(N)-K^{\prime}$.

whlle in the second case, $N^{1 / 2}-N^{1 / 2}=N^{1 / 2}\left\{\left(\frac{N}{N}\right)^{1 / 2}-1\right\}>N^{1 / 2}\left\{\left(\frac{1}{2}+b\right)^{-1 / 2 r}-1\right\}$. It follows that $t:=t(H)=\frac{1}{2}\left(H^{1 / 2}-N^{1 / 2}\right) \rightarrow-$ as $N \rightarrow \infty$ $($ or $\mathrm{H} \rightarrow \infty)$.

(Note: by $(2.17), \sigma_{1}(N)=\sigma_{1}(N(H)) \rightarrow-$ as $H \rightarrow-$, and henee $\mathrm{N}=\mathrm{N}(\mathrm{H}) \rightarrow \infty \mathrm{as} \mathrm{H} \rightarrow \bullet$.

Sett $1 \mathrm{ng}$,

$(2.20) x:=x(H)=\frac{a}{4}\left(n^{1 / 2}+n^{1 / 2}\right)^{2}$ we see that $x \rightarrow \bullet$ as $H \rightarrow \infty$, since $\mathrm{H}>\mathrm{N} \rightarrow \bullet$ as $\mathrm{H} \rightarrow \bullet$.

From $(2.20)$ we have: 


$$
\begin{aligned}
& 2 t=M^{1 / 2}-N^{1 / 2} \\
& 2 J(x / a)=n^{1 / 2}+N^{1 / 2} \\
& \text { and } 80 n^{1 / 2}=f(x / a)+t, N^{1 / 2}=J(x / a)-t
\end{aligned}
$$

Therefore,

$$
\left\{\begin{array}{l}
\left.n=(f(x / a)+t)^{2}, f f(x / a)+t\right) /(x / a)=x / a+t f(x / a) \\
N_{0}<N=(f(x / a)-t)^{2}(f(x / a)-t)(f(x / a)=x / a-t /(x / a) .
\end{array}\right.
$$

where $x$ and $t \rightarrow \infty$ as $H \rightarrow \cdots$.

In the analysis of case (A), which follows, we suppose that $N, A$. and $x$ are chosen as in $(2.17),(2.18)$, and $(2.20)$, that $t=1 / 2\left(M^{1 / 2}-N^{1 / 2}\right.$, and hence that (2.21) 1s satisfled. Clearly the cholce of $M$ and $N$ satisfy the hypothaes of Leme (2.6) and so as $x, t \rightarrow \infty$ (or $H \rightarrow \bullet$ ) we have the properties $(2.7)$ through $(2.10)$. Nov,

(2.22) $T_{1}(x):=\sum_{n=N_{0}}^{N-1} c_{n}(x) g_{n} 2-\sigma_{2}(N) \sum_{n=N_{0}}^{N-1} n^{r} c_{n}(x)$, since $\sigma_{2}(N)=\underset{n \& N}{\max }\left(-\frac{s_{n}}{n^{r}}\right)$

$$
\begin{aligned}
& \nu-\sigma_{1}(N) \sum_{n=N_{0}}^{N-1} n^{r} c_{n}(x) \text {. since } \sigma_{1}(N) \partial \sigma_{2}(N) \\
& \nu-\sigma_{1}(N) \sum_{n=N_{0}}^{N} n^{r} c_{n}(x) \text {, since } \sigma_{1}(N)>0 \\
& \left.=-\sigma_{1}(N) \text { o(1), as } x, t \rightarrow \text { or or } H \rightarrow \infty\right) .
\end{aligned}
$$

by $(2.7)$ of Lewe $(2.6)$.

Next, 1 is the least integer, N which gatisfies (2.18), so for $n=N+1, N+2, \ldots, n-1$ we have $\sigma_{n}, \frac{1}{2} \sigma_{N}=\frac{1}{2} \sigma_{1}(N)$, and trivially $\sigma_{N}=\sigma_{1}(N)>\frac{1}{2} \sigma_{1}(N)$. So for $n=N, N+1, \ldots, N-1, s_{n}, \frac{1}{2}, \sigma_{1}(N) n^{r}$.

Thus, 
(2.23) $T_{2}(x):=\sum_{n=N}^{N-1} c_{n}(x) s_{n}>\frac{1}{2} \sigma_{1}(N) \sum_{n=N}^{n-1} n^{r} c_{n}(x)-\frac{1}{2} \sigma_{1}(N)(x / a)^{r}$ as

$x, t \rightarrow-($ or $H \rightarrow \infty)$, by $(2.9)$ of Leena $(2.6)$.

Now by Leman $(2.4)$,

$s_{n}-s_{n-1} ;-k\left(n^{r+1 / 2}-(n-1)^{r+1 / 2}\right)-k \cdot(n-1)^{r}$, non, for sove positive constants $K, K^{\prime}$.

Therefore,

$s_{n}>s_{n-1}-k\left(n^{r+1 / 2}-n^{r+1 / 2}\right)-O\left(k^{r-1 / 2}\right)-k^{\prime} n^{5}$.

Now since 13 the least integer, N which satist 1 es (2.18) we have by

(2.17).

$\sigma_{n-1}>\frac{1}{2} \sigma_{N}^{\cdots}>H$ 1.e. $s_{K-1}>H(n-1)^{r}>0$.

Hence $s_{n}>H(M-1)^{r}-K\left(n^{r+1 / 2}-n^{r+1 / 2}\right)-O\left(K^{r-1 / 2}\right)-K n^{r}$.

1.e. $\left.s_{n}\right)-k\left(n^{r+1 / 2}-n^{r+1 / 2}\right)-O\left(k^{r}\right) ; n>n$.

Thus ,

(2.24) $T_{3}(x):=\sum_{n=n}^{\infty} c_{n}(x) s_{n} 2 \cdot \sum_{n=n}^{\infty}\left(-K\left(n^{r+1 / 2}-n^{r+1 / 2}\right)-0\left(n^{r}\right)\right) c_{n}(x)$

$2-K \sum_{n=n}^{\infty}\left(n^{r+1 / 2}-n^{r+1 / 2}\right) c_{n}(x)-0(1) \sum_{n=n}^{\infty} n^{r} \cdot c_{n}(x)>-o\left(x^{r}\right)-0(1) 0\left(x^{r}\right)$

$=-o\left(x^{r}\right)$ as $x, t \rightarrow-$ or $H \rightarrow-1$, by $(2.10)$ and $(2.8)$ of Leme $(2.6)$.

Consequenfly by $(2.16),(2.22),(2.23)$, and $(2.24)$,

$\frac{b(x)}{x^{r}}=\frac{T_{1}(x)}{x^{r}}+\frac{I_{2}(x)}{x^{r}}+\frac{T_{3}(x)}{x^{r}}, \sigma_{1}(N)\left(\frac{1}{2} a^{-r}-o(1)\right)-o(1) \rightarrow-\infty$

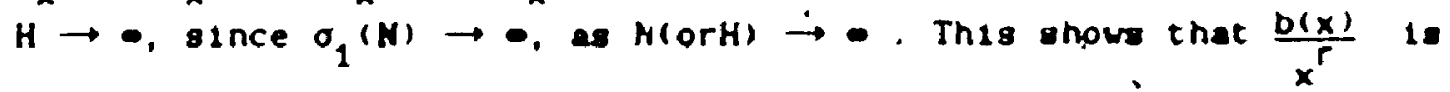
unbounded above and therefore ve have contradiction with the hypothes is $b(x)=O\left(x^{r}\right)$ as $x \rightarrow \infty$. 
Next ve suppose that case (B) holds (1.e. there exists an Mo such that $\sigma_{2}(n)>\sigma_{1}(n), n 2 H_{0}$, and that $\left\{\sigma_{n}\right\}$ is unbounded. Mis supposition 18 ruled out by an arguent siallar to the preceding one. Specifically we follow Rajagopal ([17], case (11) of Theorea 1$)$.

We first show that $\sigma_{2}(n) \leftrightarrow \bullet$ as $n \rightarrow \bullet$ :

If $\left\{\sigma_{2}(n)\right\}$ is bounded above, then there exists an $L$ such that $\sigma_{i}(n), L$ for all $n$. This saplies that $-\sigma_{n}$ ll for all $n$. On the other hand. because of case $(B)$, we have that $\sigma_{1}(n)\left(\sigma_{2}(n) \mathrm{cL}\right.$ for $n 2 H_{0}$. It followe that $\sigma_{n}$ il for all $n$. Thus $\sigma_{n}$ lot for all $n$, which is a contradiction. Hence $\left\{\sigma_{2}(n)\right\}$ is unbounded above, soreover it is increasing in $n$, therefore $\sigma_{2}(n) \rightarrow \bullet$ as $n \rightarrow \bullet$.

Now write,

$$
b(x)=\left(\sum_{n=N_{0}}^{N}+\sum_{n=N+1}^{N}+\sum_{n=N+1}^{\infty}\right) c_{n}(x) s_{n}=I_{1}(x)+T_{2}(x)+T_{3}(x) .
$$

where first $M$ and then $M$ are chosen as follows. Corresponding to any positive $H>H_{0}=\sigma_{2}\left(H_{0}\right)$, choose the least $H=M(H)$ such that

(2.25) $\quad \sigma_{2}(n)>\sigma_{1}(n), n \geqslant H, \quad \sigma_{M}=-\sigma_{2}(M)<-2 H$.

(To see that there are $H=M(H)$ such that $(2.26) 18$ satisfled consider the following: given any positive $H{ } H_{0}$ there exists an $n$ 'such that $\sigma_{2}\left(M^{\prime}\right)>2 H$, since $\sigma_{2}\left(n^{\prime}\right) \rightarrow-$ Next, $\sigma_{2}\left(H^{\prime}\right)=-\sigma_{K}$ for sose M6 $H^{\prime}$, and hence $\sigma_{2}\left(M^{\prime}\right) \nu \sigma_{2}\left(K^{\prime}\right)-\sigma_{M}=\sigma_{2}\left(M^{\prime}\right)$. Thus $-\sigma_{K}=\sigma_{2}\left(M^{\prime}\right)=\sigma_{2}\left(M^{\prime}\right)$ and $1 \mathrm{t}$ followe that $\left.-\sigma_{M}=\sigma_{2}(K)\right) 2 H$. Furthersore, $2 H>H_{0}=\sigma_{2}\left(n_{0}\right)$ implies that $\sigma_{2}(x)>\sigma_{2}\left(\mu_{0}\right)$, hence $n>\mu_{0}$ and $\left.\sigma_{2}(n)>\sigma_{1}(n), n>H_{1}\right)$

Next, choose the largest $N=N(H), N \in\left(N_{0}, H\right)$ for which (2.27) $\sigma_{N}+\frac{1}{2} \sigma_{n}=-\frac{1}{2} \sigma_{2}(n)$. (There are such $N$ when $H$ 18 large because of the following argunents. 
First, we prove the

Clale: There exists an $H_{1} 2 H_{0}$ such that $\sigma_{n}{ }^{2-H_{1}}$ for inf anftely eany values of $n$.

For the sake of a contradiction we suppose the clald is false.

Therefore, for ałl $H_{1} \gg H_{0}$. $\sigma_{n}<-H_{1}$ for all n sufflelently large, l.e. $\sigma_{n} \rightarrow-\infty$. Since there can only be finilely any values of $n$ such that $\sigma_{n}>0$, and since the actual chosce of $N_{0}$ la imaterial and satisfies $\alpha N_{0}+\beta-r>0$, it aay be increased if necessary so that o 60 . $n \partial N_{0}$, and $a N_{0}+\beta-r>0$. Now exactly as in case $(A)$ we have: $\frac{n^{r} \sigma_{n}}{\Gamma(\alpha n+\beta)}+c \frac{\sigma_{n}}{\Gamma(\alpha n+\beta-r)}, n>\beta_{0}$, where $C 18$ a positive constant.

Thus $b(x):=\sum_{n=N_{0}}^{\infty} c_{n}(x) s_{n}=a e^{-x} \sum_{n=N_{0}}^{\infty} \sigma_{n} n^{r} \frac{x^{\alpha n+\beta-1}}{\Gamma(\alpha n+\beta)}$

$$
\text { 6 } \operatorname{cac}^{-x} \sum_{n=N_{0}}^{\infty} \sigma_{n} \frac{x^{\alpha n+\beta-1}}{\Gamma(a n+\beta-r)} \text {, and nence }
$$

(2.28) $\frac{b(x)}{x^{r}} \cdot \operatorname{Cac}^{-x} \cdot \sum_{n=N_{0}}^{\infty} \sigma_{n} \frac{x^{\alpha n+\beta-r-1}}{\Gamma(\alpha+\beta-r)}$.

Since $\sigma_{n} \rightarrow-\infty$ as $n \rightarrow \infty$, total regùlarity 1 pplies that $a_{n} \rightarrow-(B, a, \beta-r)$. Hènce froa $(2.28)$ it follows that $\frac{b(x)}{x^{r}} \rightarrow-$ as $x \rightarrow \bullet$, which 13 a contradiction with the hypothesis $b(x)=O\left(x^{r}\right)$ as $x \rightarrow \cdots$

Having proven the clain we have 'that $\sigma_{n}{ }^{\partial-H_{1}}$ for infinitely any values of $n>N_{0}$. Since $\sigma_{2}(H)=\sigma_{2}(H(H))>2 H$ we have that $\sigma_{2}(n) \rightarrow-$ $\Leftrightarrow H \rightarrow \bullet$, and this implies $K=K(H) \rightarrow \infty$ as $H \rightarrow \infty$. Thus there exist $\rightarrow$ an $H_{2} \partial H_{1}$ such that for all $H \partial H_{2}, H=K(H)>H_{0}$ and $\left(H_{0}, H\right)$ contains at 'least one of the $n$ 's for which $\sigma_{n} \nu-H_{1} \nu-H>\frac{1}{2} \sigma_{n}=-\frac{1}{2} \sigma_{2}(n)$. 
In other words there are $N$ when $H$ ts large for uhich

$\left.\sigma_{H}+\frac{1}{2} \sigma_{H}=-\frac{1}{2} \sigma_{2}(H)\right)$.

By the chorce of $M$ and $N$ in $(2.26)$ and $(2.27)$,

$s_{M}-s_{N}=\sigma_{K} N^{r}-\sigma_{N} N^{r} 6 \sigma_{M} M^{r}-\frac{1}{2} \sigma_{M} N^{r}=-\sigma_{2}(M)\left(M^{r}-\frac{1}{2} N^{r}\right)$.

Further, by Lewa $(2.4)$,

$s_{H}-s_{N},-K K^{r}\left(K^{1 / 2}-N^{1 / 2}\right)-K \cdot N^{r}$. for some positive constants $K$ and $K^{\prime}$.

It follows that

$-\sigma_{2}(H)\left(H^{r}-\frac{1}{2} N^{r}\right),-K H^{r}\left(H^{1 / 2}-H^{1 / 2}\right)-K \cdot N^{r}$, and hence

$K\left(\vec{M}^{1 / 2}-N^{1 / 2}\right), \sigma_{2}(n)\left(1-\frac{1}{2}\left(\frac{N}{n}\right)^{r}\right)-K\left(\frac{N}{H}\right)^{r}, \frac{1}{2} \sigma_{2}(n)-K \rightarrow \infty$

as $\mathrm{H} \rightarrow-$. since in this case $\sigma_{2}(H) \rightarrow \infty$ as $H \rightarrow \infty$ !

That is $t:=\frac{1}{2}\left(n^{1 / 2}-N^{1 / 2}\right) \rightarrow \infty$ as $H \rightarrow \infty$.

Letting, as in case (A).

$(2.20)$

$$
x:=\frac{a}{4}\left(M^{1 / 2}+N^{1 / 2}\right)^{2} .
$$

we see that $x>(a / 4) M$, and hence $x \rightarrow-$ as $H \rightarrow \infty$.

Also, as in case (A), we obtain (2.21) from the definitions of $x$ and $t$.

We now auppose that $M, M$, and $X$ are chosen as in $(2.26),(2.27)$, and (2.20), that $t=1 / 2\left(H^{1 / 2}-N^{1 / 2}\right.$, and hence that $(2.21)$ s sat isfied.

Clearly, the cholce of $M$ and $N$ satisfy the hypothesis of lema $(2: 6)$, and 80 as $x, t \rightarrow-($ or $H \rightarrow-)$ the properties $(2.7)-(2.10)$ are all val1d.

With the cholce of $H$ in $(2.27)$ and Lema (2.4) we have for $n>h$, $s_{n}+n^{r} \sigma_{2}(n)=s_{n}-n^{r} \sigma_{n}=s_{n}-8 n^{2-K}\left(n^{r+1 / 2}-n^{r+1 / 2}\right)-K \cdot n^{r}$ for some positive constants $K$ and $K^{\prime}$.

It follow that.

$\sigma_{n}=n^{-r} a_{n}>-a^{r} n^{-r} \sigma_{2}(n)-K n^{-r}\left(n^{r+1 / 2}-n^{r+1 / 2}\right) r \cdot k \cdot n^{r} n^{-r}$ 


$$
\begin{aligned}
& =-k^{r} n^{-r}\left(\sigma_{2}(n)+k \cdot-k\left(n^{1 / 2}-n^{-r} n^{r+1 / 2}\right)\right. \\
& \left.=-1 \sigma_{2}(x)+k^{\prime}\right)-k\left(n^{1 / 2}-n^{-r} n^{r+1 / 2}\right), n 2 n^{\prime} \\
& =-t_{n} \text { say. }
\end{aligned}
$$

1.e. $-\sigma_{n}<t_{n}$ for $n>n$.

Next, $\left.-\sigma_{n}=\sigma_{2}(n)=\max _{n k}\left(-\sigma_{n}\right), 1 . e .-\sigma_{n}\right)-o_{n}$ for $n=1,2, \ldots, n$

Thus we have the following inequalitiea:

(2.29) $-\sigma_{n}<t_{n}$ tor $n>k$.

$(2.30)-\sigma_{n} 6-\sigma_{H}<t_{H}$, for $n<n$.

Since $\left\{t_{n}\right\}$ is increasing in $n$ we have for $n>d, t_{n}{ }^{2 t}$, and by $(2.29)$ :

(2.) $-\sigma_{a}<t_{m} \leqslant t_{n}$ for ninsh.

Also for $n \partial k, t_{n} t_{n}$, and by $(2.30)$ :

(2.32) $-\sigma_{E}\left\langle\tau_{n}<\tau_{n}\right.$ for ndH>E.

By $(2.32)$ and $(2.31),-\sigma_{n}<t_{n}$ for $n_{k}$ and nat. It follows fros this.

that

(2.33) $\sigma_{2}(n)<\tau_{n}$ for $n>n$, where $\sigma_{2}(n)=\max _{n}\left(-\sigma_{n}^{\prime}\right.$

Now

$T_{3}(x)=\sum_{n=M+1}^{\infty} s_{n} c_{n}(x)=\sum_{n=M+1}^{\infty} n^{r} \sigma_{n} c_{n}(x)+\sum_{n=n+1}^{\infty} \sigma_{1}(n) n^{r} c_{n}(x)$,

since $\sigma_{1}(n) 2 \sigma_{n}$.

By $(2.26)$ and $(2.33)$,

$T_{3}(x)<\sum_{n=\bar{n}+1}^{\infty} \sigma_{2}(n) n^{r} c_{n}(x)<\sum_{n=\bar{n}+1}^{\infty} t_{n} n^{r} c_{n}(x)$.

By the definftion of $t_{n}$, and (2.8) and $(2.10)$ of Leme $(2.6)$.

$v^{(2.34)} T_{3}(x)<\sum_{n=H+1}^{\infty}\left(\sigma_{2}(n)+K^{\prime}+K\left(n^{1 / 2}-n^{-r} n^{r+1 / 2}\right)\right) n^{r} c_{n}(x)$

$$
=\left(a_{2}(n)+K \cdot\right) \sum_{n=\bar{n}+1}^{\infty} n^{r} c_{n}(x)+k \sum_{n=n+1}^{\infty}\left(n^{r+1 / 2}-n^{r+1 / 2}\right) c_{n}(x)
$$




$$
=\left(\sigma_{2}(x)+K^{r}\right) \sigma\left(x^{r}\right)+K o\left(x^{r}\right)=\sigma_{2}(x) o\left(x^{r}\right)+o\left(x^{r}\right)
$$

as $x, t \rightarrow-\left(\right.$ ar $\mathrm{H}^{\circ} \rightarrow-1$ ).

Mext,

$$
\begin{aligned}
T_{1}(x) & =\sum_{n=N_{0}}^{N} c_{n}(x) a_{n}=\sum_{n=N_{0}}^{N} a_{n} n^{r} c_{n}(x), \sigma_{1}(n) \sum_{n=N_{0}}^{N} n^{r} c_{n}(x) \\
& \left(\sigma_{1}(n) \sum_{n=N_{0}}^{N} n^{r} c_{n}(x) \text {, since } N>N \text { and }\left\{\sigma_{1}(n)\right)\right. \text { is increasing. }
\end{aligned}
$$

It fallows from $(2.26)$, and $(2.7)$ of Letma $(2.6)$ that.

(2.35) $I(x)<\sigma_{2}(K) \sum_{n=N_{0}}^{N} n^{r} c_{n}(x)=\sigma_{2}(K) . o\left(x^{r}\right)$

Next, $N$ is the largest integer in (No, M) why sat isfles (2.27):

nence for $n=N+1, N+2, \ldots, n-1, \sigma_{n}<-\frac{1}{2} \sigma_{2}(n)$, and trivially

$\sigma_{M}=-\sigma_{2}(K)<-\frac{1}{2} \sigma_{2}(H)$. So for $n=N+1, n+2 \ldots, M . s_{n}<-\frac{1}{2} \sigma_{2}(n) n^{r}$.

Thue, by (2.9) of Lewne (2.6).

(2.36) $T_{2}(x)=\sum_{n=N+1}^{n} c_{n}(x) s_{n}<-\frac{1}{2} \sigma_{2}(n) \sum_{n=N+1}^{n} n^{r} c_{n}(x)$

$$
\left.\sim-\frac{1}{2} \sigma_{2}(H)(x / a)^{r} \text { as } x, t \rightarrow \infty \text { (or } H \rightarrow \infty\right) \text {. }
$$

Therefore from $(2.25)$ and using $(2.34),(2.35),(2.36)$ we have:

$\frac{b(x)}{x^{r}}=\frac{T_{1}(x)}{x^{r}}+\frac{T_{2}(x)}{x^{r}}+\frac{T_{3}(x)}{x^{r}}<-\sigma_{2}(n)\left(\frac{1}{2} a^{-r}+0(1)-o(1)\right)_{+0}(1) \rightarrow-\infty$ As $H \rightarrow \bullet$, since $\sigma_{2}(H) \rightarrow \bullet$ as $H \rightarrow \bullet$.

Th1e show that $\frac{b(x)}{x^{r}}$ is unbounded below and therefore contradicts the hypothes18 $b(x)=O\left(x^{r}\right)$ as $x \rightarrow 0$. Ruling out the supposition that case (B) holds and $\left[\sigma_{n}\right] 12$ inbounded we see that the proof $1 \mathrm{~g}$ complete. //1 
(2.37) Corollary: Given $s_{n} \in R$ such that $s_{n}-8_{n-1}=U_{L}\left(n^{r-1 / 2}\right), r 20$. and $b(x)=O\left(x^{r}\right)$ as $x \rightarrow-$, then $s_{n}=O\left(n^{r}\right)$.

47 


\section{anpte: 3}

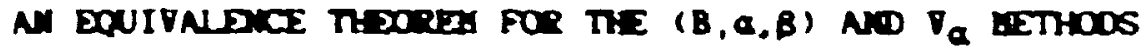

Borwein and Robinson ( $[7]$. Theores 2 ) have proved the following result: If $s_{n}-o(d n)$ then $s_{n} \rightarrow l(B, \alpha, \beta) \rightarrow s_{n} \rightarrow l\left(v_{a}\right)$

It w1l be ghown in this chapter that the result is st:ll true when the condition $s_{n}$-o $\left(f^{n}\right)$ is replaced by $s_{n}=d_{(n}{ }^{\prime}$, , r do. In order to counter the effect of this videning of the order condition, a much closer approxiation to the function $\frac{a^{-x} x^{\alpha p+\beta-1}}{\Gamma(a n+\beta)}$ is required than 18 given in Lems (2.1) and this 18 the point of the functions $8_{k}$ and $R_{k}$ introduced in Lema (3.8). The proof of Leme (3.8) 18 gialiar to one given by Faulhaber ( $(10]$, proof of Hilfsatz $S)$. The proof of our equivalence theorem, between the $(B, \alpha, \beta)$ wethod and the Yathod, 18 along the I1nes of ([10], Satz II).

(3.1) Lem: (gee Hyslop ( (14), Lema 1)) If $p ; r 20,3 / 2<\left[<1, h_{n}=n-x / \alpha\right.$, then as $x \rightarrow 0$,

(3.2)

$$
\sum_{n>x / a+x^{5}} n^{r} n_{n}^{p} \exp \left(-\frac{a^{2} n_{n}^{2}}{2 x}\right)=o(1) .
$$

(3.3)

$$
\sum_{0 \times n \times / a-x^{5}} n^{r} \ln _{n} 1^{p} \exp \left(-\frac{a^{2} n_{n}^{2}}{2 x}\right)=0(1)
$$

$$
\sum_{n=0}^{\infty} n^{r}\left|h_{n}\right|^{p} \exp \left(-\frac{a^{2} n_{n}^{2}}{2 x}\right)=O\left(x^{r+1 / 2(p+1)}\right) .
$$

Proof: For (3.2), $s(x):=\sum_{n>x / a+x} n^{n^{r} n_{n}^{p}} \exp \left(-\frac{a^{2} n_{n}^{2}}{2 x}\right)$ 
$=\left\{\sum_{x / a+x} \sum_{<n<2 x / a}+\sum_{n>2 x / a}\right\} n^{r} n_{n}^{p} \exp \left(-\frac{a^{2} n_{n}^{2}}{2 x}\right)$

$-(2 x / a)^{r} \sum_{x / a+x^{2}} \sum_{(n<2 x / a}^{-} n_{n}^{p} \exp \left(-\frac{a^{2} n_{n}^{2}}{2 x}\right)+2^{r} \sum_{n>2 x / a} n_{n}^{p+r} \exp \left(-\frac{a^{2} n_{n}^{2}}{2 x}\right)$.

since for $n 22 x / a$, no $2(n-x / a)=2 n_{n}$.

Theretore

$$
\begin{aligned}
s(x) & \left.=\left\{x^{r} \sum_{x / a+x^{2}<n<2 x / a} n_{n}^{p} \exp \left(-\frac{a^{2} n_{n}^{2}}{2 x}\right)\right\}+u \sum_{n>2 x / a} n_{n}^{p+r} \exp \left(-\frac{a^{2} n_{n}^{2}}{2 x}\right)\right\} \\
& =o\left(x^{r} s_{1}(x)\right)+o\left(s_{2}(x)\right) .
\end{aligned}
$$

Now, $s_{1}(x)=\sum_{n=n_{0}}^{n_{1}} n_{n}^{p} \exp \left(-\frac{a^{2} n_{n}^{2}}{2 x}\right)$, where $n_{0}$ is the sadlest

integer $>x / a+x^{2}$, and $n$, is the largest integer $<2 x / a$.

Let $f(y)=y^{p} \exp \left(-\frac{a^{2} y^{2}}{2 x}\right)$. Then $f(y)$ decreases for $y, f(p x) / a$. For $x$ sufficlently large and by our cholce of no the inequalily $J(p x) / a<x^{5}<n_{0}-x / a=n_{n_{0}} 18$ valld. Apply1ng Leena (1.6) we have:

$$
\begin{aligned}
& s_{1}(x), n_{n_{0}}^{p} \exp \left(-\frac{a^{2} n_{n_{0}}^{2}}{2 x}\right)+\int_{n^{2}}^{n_{n_{0}}} y^{p} \exp \left(-\frac{a^{2} y^{2}}{2 x}\right) d y \\
& \qquad x^{\sum p_{n_{1}}} \exp \left(-\frac{a^{2} x^{2 \xi}}{2 x}\right)+\int_{x^{c}}^{x / a} y^{p} \exp \left(-\frac{a^{2} y^{2}}{2 x}\right) d y, \operatorname{since} n_{n_{1}}=n_{1}-x / a<x / a
\end{aligned}
$$

and $n_{n_{0}}=n_{0}-x / a>x^{E}$.

Since the integrand decreases on the interval $\left(x^{t}, x / a\right)$, where' $x$ is 


$$
\begin{aligned}
& \text { large and } 1 / 2<1<1 \text {, 1t forlows that } \\
& \left.x^{r} s_{1}(x)=\alpha\left\{x^{\sum p+r} \exp \left(-\frac{a^{2} x^{2 \varepsilon-1}}{2}\right)\right\}+\mathcal{O} x^{r}\left(x / a-x^{2}\right) x^{\sum p} \exp \left(-\frac{a^{2} x^{2 \varepsilon-1}}{2}\right)\right\} \\
& =0(1) \text { as } x \rightarrow-\text {. since } 2 \xi-1>0 \text {. }
\end{aligned}
$$

For the sum $s_{2}(x)$, let $n_{0}$ be the saballest integer, $2 x / a$ and $f(y)=y^{p+r} \exp \left(-\frac{a^{2} y^{2}}{2 x}\right)$. The function $f(y)$ decreases for $p / d(p+r) / x / \alpha$.

For $x$ sutficientiy large and by our chosce of $n_{0}$ the inequality

$d(p+r) d x / \alpha, x / a, n_{0}-x / a=n_{n_{0}}$ is valid. In particular $f(x)$

. decreases on the anterval $\left[n_{n_{0}}, n_{0}\right]$, where $\rightarrow n_{0}$.

By Leama (1.6),

$t_{\square}:=\sum_{n=n_{0}}^{E} n_{n}^{p+r} \exp \left(-\frac{a^{2} n_{n}^{2}}{2 x}\right): n_{n_{0}}^{p+r} \exp \left(-\frac{a^{2} n_{n_{0}}^{2}}{2 x}\right)+\int_{n_{n_{0}}}^{n_{m}} y^{p+r} \exp \left(-\frac{a^{2} y^{2}}{2 x}\right) d y$ $r(x / \alpha)^{p+r} \exp \left(-\frac{a^{2}}{2 x}(x / \alpha)^{2}\right)+\int_{x / a}^{\infty} y^{p+r} \exp \left(-\frac{a^{2} y^{2}}{2 x}\right) d y$.

since $n_{n_{0}}=n_{0}-x / \alpha 2 x / \alpha$.

Since [ $t_{a}$ ls 1 ncreasing and bounded above we have:

$s_{2}(x)=\sum_{n=n_{0}}^{\infty} n_{n}^{p+r} \exp \left(-\frac{a^{2} h_{n}^{2}}{2 x}\right) \leqslant(x / a)^{p+r} \exp (-x / 2)+\int_{x / \alpha}^{\infty} y^{p+r} \exp \left(-\frac{a^{2} y^{2}}{2 x}\right) d y$

$=0\left(x^{p+r} \exp (-x / 2)\right)+x^{1 / 2(p+r+1)} \int_{d x / \alpha}^{\infty} u^{p+r} \exp \left(-\frac{a^{2} u^{2}}{2}\right) d u$.

where $u^{2}=\frac{y^{2}}{x}$

To find an estiaste t or the integral, let $\lambda=2(p+r+2)$ and $f(u)=u^{-\lambda} \exp \left(\frac{a^{2} u^{2}}{2}\right)$ for $u \in[\sqrt{ } x / \alpha,-)$. f(u) increases for $u$ w $\lambda / \alpha$ so it 
certainlv increases for $u d d x / a$. Where $x$ 19 large. That is $f(u) d f(d x / a)$ for $u \in(\delta x / a,-1)$ L.e. $\left.\exp \left(-\frac{a^{2} u^{2}}{2}-\right),(a u)^{-\lambda} x^{x / 2} \exp -x / 2\right)$ for $u \in(d x / a, \infty)$.

Thus.

$$
\begin{aligned}
\int_{\delta x / a}^{\infty} u^{p+r} \exp \left(-\frac{a^{2} u^{2}}{2}\right) d u & =\left\{x^{x / 2} \exp (-x / 2) \int_{J x / a}^{\infty} u^{n+r-1} d u\right\} \\
& =\left\{x^{1 / 2(p+r+1)} \exp (-x / 2)\right\} .
\end{aligned}
$$

since $t=2(p+r+2)$.

It follows that.

$\begin{aligned} s_{2}(x)=\sum_{n=n_{0}}^{\infty} h_{n}^{p+r} \exp \left(-\frac{a^{2} h_{n}^{2}}{2 x}\right) & =o\left(x^{p+r} \exp (-x / 2)\right)+o\left(x^{p+r+1} \exp (-x / 2)\right) \\ & =o(1) \text { es } x \rightarrow \infty .\end{aligned}$

$F \operatorname{lnally}, S(x)=o\left(x^{r} S_{1}(x)\right)+o\left(S_{2}(x)\right)=o(1)+o(1)=o(1)$ as $x \rightarrow-$

To prove (3.3) it suffices to show that

$s(x):=\sum_{0 r n<x / \alpha-x^{2}} n^{r} k_{n}^{p} \exp \left(-\frac{\alpha^{2} k_{n}^{2}}{2 x}\right)=o(1)$ as $x \rightarrow$, where $k_{n}=x / \alpha-n$.

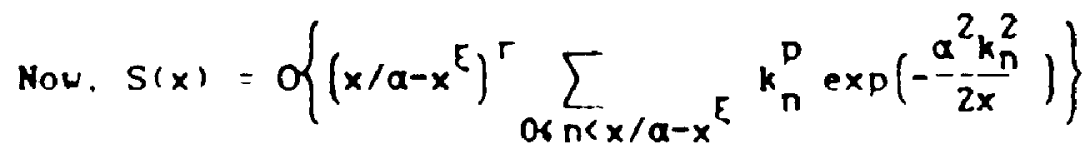

$$
=\left\{\left(x / \alpha-x^{E}\right)^{r} \sum_{n=0}^{n_{1}} k_{n}^{p} \exp \left(-\frac{\alpha^{2} k_{n}^{2}}{2 x}\right)\right\} \text {, where } n, 18 \text { the largest }
$$

integer $<x / \alpha-x^{E}$.

The function $f(y)=y^{p} \exp \left(-\frac{a^{2} y^{2}}{2 x}\right)$ decreases for $y, f(p x) / a$. For $x$ suffletently large and $\left(\sum>1 / 2\right.$, the inequallty $f(p x) / a<x^{5}+x / a-n_{i}=k_{n_{1}}$ 
52

$18 \mathrm{vel} 1 \mathrm{~d}$.

By Lem (1.7) with $n_{0}=0$.

$$
\begin{aligned}
\sum_{n=0}^{n_{1}} k_{n}^{p} \exp \left(-\frac{a^{2} k_{n}^{2}}{2 x}\right) & \cdot k_{n_{1}}^{p} \exp \left(-\frac{a^{2} k_{n_{1}}^{2}}{2 x}\right)+\int_{k_{n_{1}}}^{k_{0}} y^{p} \exp \left(-\frac{a^{2} y^{2}}{2 x}\right) d y \\
& \cdot x^{k p} \exp \left(-\frac{a^{2} x^{2 k-1}}{2}\right)+\int_{x^{2}}^{x / a} y^{p} \exp \left(-\frac{a^{2} y^{2}}{2 x}\right) d y .
\end{aligned}
$$

since $k_{0}=x / \alpha$ and $k_{n_{1}}=x / a-n_{1}>x^{\xi}$.

Since the integrand decreases on the interval $\left[x^{E}, x / a\right)$, where $x$ is large and $1 / 2<E<1$, it follow that

$$
\begin{aligned}
S(x) & \left.\left.=\alpha\left(x / a-x^{\varepsilon}\right)^{r} x^{L p} \exp \left(-\frac{a^{2} x^{2 k-1}}{2}\right)\right\}+\alpha\left(x / a-x^{\varepsilon}\right)^{r+1} x^{\varepsilon p} \exp \left(-\frac{a^{2} x^{2 E-1}}{2}\right)\right\} \\
& =0(1) \text { as } x \rightarrow-\text { since } 2 \xi-1>0 .
\end{aligned}
$$

To prove (3.4) write, $s(x):=\sum_{n=0}^{\infty} n^{r}\left|h_{n}\right|^{p} \exp \left(-\frac{a^{2} n_{n}^{2}}{2 x}\right)$

$$
\begin{aligned}
& =\left.\left\{\sum_{0<n<2 x / a}+\sum_{n>2 x / \alpha}\right\} n^{r} \ln _{n}\right|^{p} \exp \left(-\frac{a^{2} n_{n}^{2}}{2 x}\right) \\
& =s_{1}(x)+s_{2}(x) .
\end{aligned}
$$

Recalling the proof of $(3.2)$ we see that $S_{2}(x)=0(1)$ as $x \rightarrow \rightarrow$

Next, setting $k_{n}=-n_{n}$ we have:

i

$$
\left.s_{1}(x), 12 x / \alpha\right)^{r} \sum_{\alpha<n<2 x / \alpha}\left|h_{n}\right|^{p} \exp \left(-\frac{\alpha^{2} n_{n}^{2}}{2 x}\right)
$$




$$
\begin{aligned}
& \text { 6 }(2 x / a)^{r}\left\{\sum_{a, n \sigma x / a} k_{n}^{p} \exp \left(-\frac{a^{2} k_{n}^{2}}{2 x}\right)+\sum_{x / a<n<2 x / a} n_{n}^{p} \exp \left(-\frac{a^{2} n_{n}^{2}}{2 x}\right)\right\} \\
& =(2 x / a)^{r}\left(s_{1,1}(x)+s_{1,2}(x)\right) .
\end{aligned}
$$

For the sum $S_{1,1}(x)$. Let $n_{1}$ be the largest integer $x / a$, so that

$s_{1,1}(x)=\sum_{n=0}^{n_{1}} k_{n}^{p} \exp \left(-\frac{a^{2} k_{n}^{2}}{2 x}\right)$

Let $f(y)=y^{p} \exp \left(-\frac{a^{2} y^{2}}{2 x}\right)$. Then $f(y)$ increases for $0 ; y f(p x) / a$,

decreases for $y \partial f(p x) / a$, and attains 1 ts $\max 1 \mathrm{au}$ at $y=\sqrt{ }(p x) / a$. By the chozce of $n_{1}, k_{n_{1}}=x / \alpha-n_{1}>0$ so in particular $f(y)$ increases on

$\left[k_{n_{1}}, k_{x_{0}}\right]$, and decreases on $\left[k_{x_{0}}, k_{0}\right]$, where $k_{x_{0}}=x / \alpha-x_{0}=J(p x) / \alpha$ and $k_{0}=x / a$.

By Lem (1.9) with $n_{0}=0$.

$$
\begin{aligned}
s_{1,1}(x) & k_{x_{0}}^{p} \exp \left(-\frac{a^{2} k_{x_{0}}^{2}}{2 x}\right)+\int_{k_{n_{1}}}^{k} y^{p} \exp \left(-\frac{a^{2} y^{2}}{2 x}\right) d y \\
& \cdot\left(\frac{v(p x)}{\alpha}\right)^{p} \exp \left(-\frac{a^{2} p x}{2 a^{2}}\right)+\int_{0}^{x / a} y^{p} \exp \left(-\frac{\alpha^{2} y^{2}}{2 x}\right) d y \\
& =0\left(x^{p / 2}\right)+\int_{0}^{x / \alpha} y^{p} \exp \left(-\frac{\alpha^{2} y^{2}}{2 x}\right) d y, \text { since } k_{n_{1}} 20 .
\end{aligned}
$$

Next.

$s_{1,2}(x)=\sum_{n=n_{0}}^{n_{1}} n_{n}^{p} \exp \left(-\frac{\dot{\alpha}^{2} n_{n}^{2}}{2 x}\right)$, where $n_{0} 18$ the sallest
1nteger $x / \alpha_{1}$ and $n_{1}$ ia the largest 1nteger $<2 x / \alpha$.

Defining $f$ above, and letting $h_{x_{0}}=x_{0}-x / \alpha=f(p x) / \alpha$ we $f$ ind by 
applying Leme (1.8) that,

$s_{1.2}(x) \cdot n_{x_{0}}^{p} \exp \left(-\frac{a^{2}}{2 x} n_{x_{0}}^{2}\right)+\int_{n_{n_{0}}}^{n_{n_{1}}} y^{p} \exp \left(-\frac{\alpha^{2} v^{2}}{2 x}\right) d v$
\[ o\left(x^{p / 2}\right)+\int_{0}^{x / \alpha} v^{p} \exp \left(-\frac{a^{2} y^{2}}{2 x}\right) d y \text {, s1nce } n_{n_{1}}=n_{1}-x / \alpha<x / \alpha \text { and } \]
$n_{n_{0}=n_{0}-x / \alpha>0 .}$

Therefore. $s_{1}(x)\left(r(2 x / \alpha)^{\top}\left(s_{1,1}(x)+s_{1,2}(x)\right)\right.$

$=\left\{x^{r} \int_{0}^{x / \alpha} y^{P} \exp \left(-\frac{a^{2 y^{2}}}{2 x}\right) d y\right\}+O\left(x^{r+p / 2}\right)$

$=\left\{x^{r+1 / 2(p+1)} \int_{0}^{\infty} u^{1 / 2(p-1)} \exp (-u) d u\right\}+o\left(x^{r+p / 2}\right)$

$=\left\{x^{r+1 / 2(p+1)}\right\}$.

Finally, $\left.s(x)=s_{1}(x)+s_{2}(x)=0(1)+\alpha x^{r+1 / 2(p+1)}\right\}$ As $x \rightarrow \infty$

$$
\left.=\alpha x^{r+1 / 2(p+1)}\right\} .111
$$

Definition ( $(2)$ or $(3))$ : A sequence $\left[s_{n}\right\}$ is sald to be of finite order if $a_{n}=0\left(n^{c}\right.$, for some finite $c$.

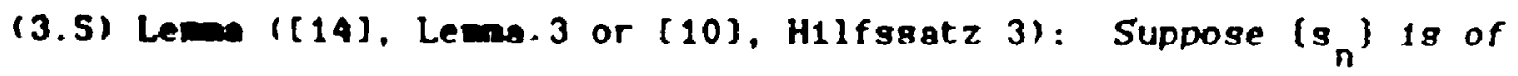
finite order. Then for $a>0$ and $q>0$,

$\sum_{n=0}^{\infty} s_{n} \exp \left(-\frac{a(n-x)^{2}}{2 x}\right)=0\left(x^{1 / 2+2+a, n s} x \rightarrow\right.$ 
1anlles for each integer g>0, and for each $n>0$

$\sum_{n=0}^{\infty} s_{n}(n-x)^{j} \exp \left(-\frac{a(n-x)^{2}}{2 x}\right)=o\left(x^{1 / 2(j+1)+q+n}\right)$ as $x \rightarrow \cdot$

Recalling that $h_{n}=n-x / a$, 1t 18 obvious that the following result follows from the previous one.

(3.6) Lema: Supptge $\left\{s_{n}\right\}$ is of finite order. Then for $a>0$ and $a>0$ $\sum_{n=0}^{\infty} s_{n} \exp \left(-\frac{a^{2} n_{n}^{2}}{2 x}\right)=o\left(x^{1 / 2+a}\right)$ as $x \rightarrow \infty$ Implles for each 1nteget $y>0$, and each $n>0$

$\sum_{n=0}^{\infty} s_{n} n_{n}^{j} \exp \left(-\frac{a^{2} n_{n}^{2}}{2 x}\right)=0\left(x^{1 / 2(j+1)+a+n}\right)$ as $x \rightarrow \infty$

(3.7) Leme ([14], Theorem 2 or $[10]$, Hilfsgatz 4$)$ :

Suppose $\left[s_{n}\right\}$ is of finite order. Then

$\sum_{k=0}^{\infty} \dot{s}_{k} \exp \left(-\frac{\alpha(n-k)^{2}}{2 n}\right)=0\left(n^{1 / 2}\right.$, ss $n \rightarrow \infty, n=1,2, \ldots$

1 mplies

$\sum_{k=0}^{\infty} s_{k} \exp \left(-\frac{a(x-k)^{2}}{2 x}\right)=o\left(x^{1 / 2}\right.$, ss $x \rightarrow \cdots$

The next lewa 18 obtalned by following the arguments given by 
Faulhaber( $(10)$, Hilfssatz 5$)$. For another simllar result see Hvs lop ( $(14\}$, Lema 5$)$.

(3.8) Lems: If $O<H<1,(1-H) \times / a<n<(1+H) \times / \alpha$, and $k$ is any integer , $\overrightarrow{2}$, then $a s \times \rightarrow$.

$$
c_{n}(x)=\alpha / f(2 \pi x) \exp \left(-\frac{a^{2} n_{n}^{2}}{2 x}+g_{k}+R_{k}\right)
$$

where $n_{n}=n-x / \alpha, R_{k}=\left\{\frac{\left|n_{n}\right|^{k+1}+1}{x^{k}}\right\}, B_{k}=\sum_{1=1}^{k} \sum_{j=0}^{1+1} b_{1}, 1 \frac{n_{n}^{j}}{x^{1}}$, and

$b_{1, j}(1616 k$, or $j(1+1)$ are constants depending on $a$ and $B$. In $\underset{\hat{s}}{\operatorname{particular}} b_{1,2}=b_{k, k+1}=0$.

Proof: the have the following form of Sttrling's formula:

(3.9) $\log \Gamma(z+\beta)=(z+\beta-1 / 2) \log z-z+1 / 2 \log (2 \pi)+$

$\sum_{r=1}^{k-i} \frac{(-1)^{r+1} B_{r+1}(B)}{r(r+1) z^{r}}+o\left(z^{-k-1}\right), \quad k=1,2 \ldots|\arg z|<\pi$.

(See $[1]$, p. 48 , equat ion 12.)

Here $B_{r+1}(B)(16 r(k)$ are the Bernoull polynomials. For example $B_{2}(B)=B^{2}-B+1 / 6, B_{3}(B)=\beta^{3}-3 / 2 B^{2}-1 / 2 B_{1} \ldots$.

Setting $z=\alpha n$, and $h_{n}=n-x / \alpha$ in $(3.9)$ we have:

(3.10)

$108 r(a n+\beta)=\left(a n_{n}+x+\beta-1 / 2\right) \log x+\left(\alpha n_{n}+x+\beta-1 / 2\right) \log \left(\frac{\alpha h_{n}}{x}+1\right)$

$$
-\operatorname{ah}_{n}-x+1 / 2 \log (2 \pi)+\sum_{r=1}^{k} \frac{(-1)^{r+1} B_{r+1}(B)}{r(r+1) x^{r}}\left(\frac{a h_{n}}{x}+1\right)^{-r} .
$$


57

$$
+\left\{x^{-k-1}\left(\frac{a h_{n}}{x}+1\right)^{-k-1}\right\}, \quad \text { for } k=1,2, \ldots
$$

Since $(1-H) \times / \alpha<n<(1+H) \times / \alpha, 0<H<1$, then $1-H<\frac{a h_{n}}{x}+1<1+H=$ and $1 \frac{a h_{n}}{x} \mid s H<1$. It follows that

(3.11) $x^{-k-1}\left(\frac{a h_{n}}{x}+1\right)^{-k-1}=0\left(x^{-k-1}\right)=O\left(x^{-k}\right)$.

Moreover.

(3.12) $\left.\left(\frac{a h_{n}}{x}+1\right)^{-r}=\sum_{j=0}^{k-r}\left(\begin{array}{c}-r \\ j\end{array}\right)\left(\frac{a h_{n}}{x}\right)^{j}+\alpha\left(\frac{\left|h_{n}\right|}{x}\right)^{k-r+1}\right\}$.

and

(3.13) $\log \left(\frac{\alpha h_{n}}{x}+1\right)=\sum_{j=1}^{k}\left(\frac{-1)^{j-1}}{j}\left(\frac{\alpha h_{n}}{x}\right)^{j}+o\left\{\left(\frac{\left|h_{n}\right|}{x}\right)^{k+1}\right\}\right.$.

by Remark (1.2) with $\frac{a h_{n}}{x}$ in place of $x$.

From (3.12) we have:

(3.14) $\sum_{r=1}^{k} \frac{(-1)^{r+1} B_{r+1}(B)}{r(r+1) x^{r}}\left(\frac{a h_{n}}{x}+1\right)^{-r}$.

$$
\begin{aligned}
& =\sum_{r=1}^{k} \sum_{j=0}^{k-r} \frac{(-1)^{r+1} B_{r+1}(\beta)}{r(r+1)}\left(\begin{array}{c}
-r \\
j
\end{array}\right) \frac{\left(a h_{n}\right)}{x^{r+j}} \\
& +\sum_{r=1}^{k} \frac{(-1)^{r+1} B_{r+1}(\beta)}{r(r+1) x^{r}}\left\{\left(\frac{\left|h_{n}\right|}{x}\right)^{k-r+1}\right\} \\
& =\sum_{r=1}^{k} \sum_{j=0}^{k-r} d_{r, j} \frac{h_{n}^{j}}{x^{r+j}}+\sum_{r=1}^{k} \frac{1}{x^{r}}\left\{\left(\frac{\left|h_{n}\right|}{x}\right)^{k-r+1}\right\}=t_{k}+u_{k},
\end{aligned}
$$

where $d_{r, j} 18$ some constant which depends on $a$ and $B$. 
Since $\left|n_{n}\right|^{k-r+1},\left|n_{n}\right|^{k+1}+1$ for $16 r 6 k$, it follows that

$$
u_{k}=0\left\{\frac{\left|n_{n}\right|^{k+1}+1}{x^{k+1}}\right\}=0\left\{\frac{\left|n_{n}\right|^{k+1}+1}{x^{k}}\right\} \text { as } x \rightarrow \infty .
$$

As for the sue $t_{k}$, Inverting the sumption we gee that

$$
t_{k}=\sum_{j=0}^{k-1} \sum_{r=1}^{k-j} d_{r, j} \frac{n_{n}^{j}}{x^{r+j}} .
$$

Letting $r+j=1, t_{k}=\sum_{j=0}^{k-1} \sum_{1=1+j}^{k} d_{1-j, j} \frac{n_{n}^{j}}{x^{1}}$.

Inverting once again we have:

(3.15) $t_{k}=\sum_{j=1}^{k} \sum_{j=0}^{1-1} d_{1-j, j} \frac{n_{n}^{j}}{x^{1}}$.

Setting $R_{k}=\left\{\frac{\left|h_{n}\right|^{k+1}+1}{x^{k}}\right\}$, it follows from (3.14) that

(3.16) $\sum_{r=1}^{k} \frac{(-1)^{r+1} B_{r+1}(\beta)}{r(r+1) x^{r}}\left(\frac{a h_{n}}{x}+1\right)^{-r}=t_{k}+R_{k}$ as $x \rightarrow \infty$.

Substituting $(3.11),(3.13)$, and $(3.16)$ into $(3.10)$ we have:

$$
\begin{aligned}
\log r(\alpha n+\beta) & =\left(\alpha h_{n}+x+\beta-1 / 2\right) \log x-\alpha h_{n}-x+1 / 2 \log (2 \pi) \\
& +\left(\alpha h_{n}+x+\beta-1 / 2\right)\left\{\sum_{j=1}^{k} \frac{(-1)^{j-1}}{j}\left(\frac{a h_{n}}{x}\right)^{j}+\left\{\left(\frac{\operatorname{lh} h_{n}}{x}\right)^{k+1}\right\}\right\} \\
& +t_{k}+R_{k}, \quad x \rightarrow-, k=1,2, \ldots .
\end{aligned}
$$


Consequent ly, for $x \rightarrow \bullet, k=1,2, \ldots$

(3.17)

$$
\begin{aligned}
& \log c_{n}(x)=\log \alpha-x+(\alpha n+\beta-1) \log x-\log \Gamma(\alpha n+\beta) \\
& =\log \alpha-x+(a n+\beta-1) \log x-(a n+\beta-1 / 2) \log x \\
& -\left(a h_{n}+x+B-1 / 2\right)\left\{\sum_{j=1}^{k} \frac{(-1)^{j-1}}{j}\left(\frac{a h_{n}}{x}\right)^{j}+\left\{\left(\frac{\left|h_{n}\right|}{x}\right)^{k+1}\right\}\right\} \\
& +a h_{n}+x-1 / 2 \log (2 \pi)-t_{k}+R_{k} \\
& =\log (\alpha / J(2 \pi x))+a h_{n}+\left(\alpha h_{n}+x+\beta-1 / 2\right) \sum_{j=1}^{k} \frac{(-1)^{j}}{j}\left(\frac{\alpha h_{n}}{x}\right)^{j} \\
& -t_{k}+o\left\{h_{n}\left(\frac{\left|h_{n}\right|}{x}\right)^{k+1}\right\}+o\left\{x\left(\frac{\left|h_{n}\right|}{x}\right)^{k+1}\right\}+o\left\{\left(\frac{\left|h_{n}\right|}{x}\right)^{k+1}\right\}+R_{k} \text {. }
\end{aligned}
$$

Next, $\left(\frac{\left|h_{n}\right|}{x}\right)^{k+1}=\left\{\frac{\left|h_{n}\right|^{k+1}}{x^{k}}\right\}$ as $x \rightarrow \infty$. and since $\frac{\left|h_{n}\right|}{x} \cdot \frac{H}{a}$ it.

follows that $h_{n}\left(\frac{\left|h_{n}\right|}{x}\right)^{k+1}=\left\{\frac{\left|h_{n}\right|^{k+1}}{x^{k}}\right\}$.

It 18 clear that all of the O-terms as well as the term $\frac{(-1)^{k}}{k} \frac{\left(a h_{n}\right)^{k+1}}{x^{k}}$ on the right side of (3.17) may be incorporated in $\mathbf{R}_{\mathbf{k}}$.

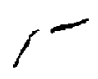

$$
\text { It follows from }(3.17) \text {, that for } k=2,3, \ldots \text { and } x \rightarrow \infty \text {, }
$$

(3.18) $\left.\log c_{n}(x)=\log (\alpha / 2 \pi x)\right)-\frac{a^{2} n_{n}^{2}}{2 x}+\sum_{j=3}^{k}(-1)^{j}\left(\frac{1}{j}-\frac{1}{j-1}\right) \frac{\left(a h_{n}\right)^{j}}{x^{j-1}}-$

$$
+(\beta-1 / 2) \sum_{j=1}^{k} \frac{(-1)^{j}}{j}\left(\frac{\alpha h_{n}}{x}\right)^{j}-t_{k}+R_{k}:
$$


where the first sumation on the right side $1 \mathrm{~s}$ zero for $k=2$.

(Note: from (3.17) with $k=1$ we see, after the term $-\frac{\left(a h_{n}\right)^{2}}{x}$ and the O-terws have been absorbed in $R_{1}$, that

$$
\left.\log c_{n}(x)=\log (\alpha /(2 \pi x))-(\beta-1 / 2) \frac{\alpha h_{n}}{x}-t_{1}+R_{1}\right)
$$

Recailing the definition of $t_{k}$, given by (3.15), it 18 clear that the entire sum:

$\sum_{j=3}^{k}(-1)^{j}\left(\frac{1}{j}-\frac{1}{j-1}\right) \frac{\left(a h_{n}\right)^{j}}{x^{j-1}}+(\beta-1 / 2) \sum_{j=1}^{k} \frac{(-1)^{j}}{j} \cdot\left(\frac{a h_{n}}{x}\right)^{j}-t_{k}$

$B_{k}=\sum_{1=1}^{k} \sum_{j=0}^{1+1} b_{1, j} \frac{n_{n}^{j}}{x^{1}}$, where in particular $b_{1,2}=b_{k, k+1}=0$.

It followe from (3.18) that for $k=2,3, \ldots$ and $x \rightarrow-$,

$\log c_{n}(x)=\log (a / \sqrt{ }(2 \pi x))-\frac{a^{2} h_{n}^{2}}{2 x}+g_{k}+R_{k}$.

The leme follows. ///.

(3.19), Reeark: Lema (3.8) 18 also valld for all $n$ such that $\left|h_{n}\right|=|n-x / a| 6 x^{5}, 1 / 2<\varepsilon<2 / 3$, because the inequal $1 t y$ :

$(1-H) x / \alpha<x / a-x^{5} \leqslant n<x / \alpha+x^{E}<(1+H) x / \alpha$ is certainly true for $x$ sufficient ly large.

(3.20) Reearks in the proof of the theoren which follows we will be interested in the for of $\varepsilon_{k}^{\mu}$, where $i j$ is positive integer. Since $\checkmark$. 
$g_{k}=\sum_{1=1}^{k} \sum_{j=0}^{1+1} b_{1, j} \frac{h_{n}^{j}}{x^{1}}$, where $b_{1,2}=0$, we can expand $g_{k}^{\mu}$ as a sum of a finite number of terms of the form $\vec{n}_{1, j} \frac{n_{n}^{3}}{x^{1}}$. Our purpese is to deteraine the values of 1 and $J$ sppearing in this expansion. First, observe that in the expansion of $g_{k}$. the lovest pover of $x^{-1}$ 131 and the highest $1 \mathrm{~s} k$. Hence in the expansion. of $g_{k}^{\prime \prime}$, the lovest

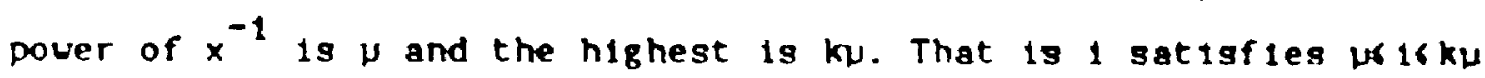
in the expansion of $g_{k}^{\mu}$. Next, to determine the values of $j$ in the expansion of $g_{k}^{\mu}$, write $g_{k} \mu$ times as

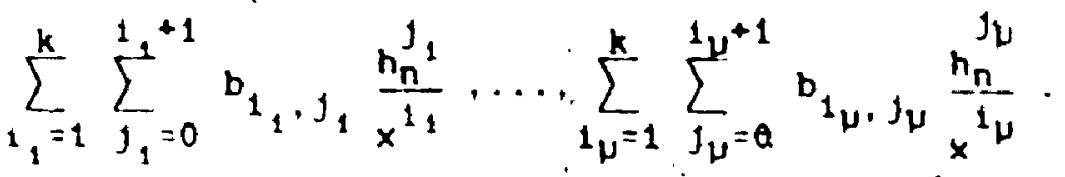

When we consider $g_{k}^{\nu}$ we multiply, terms of the form $b_{1_{1}, j_{1}} \frac{n_{n}^{j}}{x_{1}^{1}} \ldots$ $b_{1_{\nu}, f_{\mu}} \frac{h_{n}^{j_{\mu}}}{x^{1_{\mu}}}$ to obtain ter of the form $\bar{b}_{1, j} \frac{n_{n}^{j}}{x^{1}}$ where. $1=\sum_{r=1}^{\mu} 1_{r}, t=\sum_{r=1}^{k L} f_{r}$ and $\bar{b}_{1, j}=\prod_{r=1}^{\mu} b_{1_{r}, f_{r}}$

Since of $g_{r^{6}} 1_{r}+1, r=1,2, \ldots$, ve have that or $j 6 \sum_{r=1}^{k}\left(1_{r}+1\right)=1+n$.

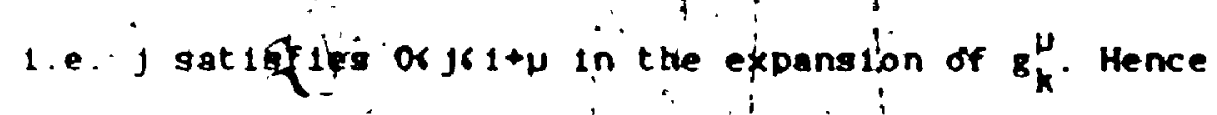
$B_{k}^{\mu}=\sum_{1 \times p}^{k \nu} \sum_{j=0}^{1+\mu} \vec{b}_{1, j} \sum_{k j}^{j}$ Hovever, $\bar{b}_{\mu, j}=0$ for $2 p$ jhy. To see this we write, as above, 
$\vec{b}_{\mu, 1}=\prod_{r=1}^{\mu} b_{1_{r}+j_{r}}$ where $\mu=\sum_{r=1}^{\mu} 1_{r}, 2 \mu>j=\sum_{r=1}^{\nu} j_{r}>\mu$. and $1_{r}>1$.

or $J_{r^{\prime}} 1_{r}+1$ for $r=1,2, \ldots, \mu$. It 13 imediate that $1_{r}=1, r=1,2, \ldots, \mu$ and hence or jro2, $r=1,2, \ldots, \mu$. Froe the condition $\sum_{r=1}^{N} j_{r}$, $\nu$ le not follows that at least one $j_{r}=2$. Thus $b_{1,}, j_{r}=b_{1,2}=0$ and $b_{\mu, g}=0$, for $J>N$. Hence

$g_{k}^{\mu}=\sum_{j=0}^{\mu} \bar{b}_{\mu, j} \frac{n_{n}^{j}}{x^{\mu}} \cdot \sum_{1=\mu+1}^{k \mu} \sum_{j=0}^{1+\mu} \bar{b}_{1, \delta \frac{n_{n}^{j}}{x^{1}}}^{j}$. In other woras $g_{x}^{\mu}$ is a finite sun of teras of the fora $\bar{b}_{1, j} \frac{n_{n}^{j}}{x^{1}}$ with values 1 and, set 1sfying or $j \mu$ for $1=\mu$, and or $j 61+\mu$ for $13 \mu+1$.

(3.21) Theorea: Suppose $s_{n}=O\left(n^{r}\right)$, r 20 , then $s_{n} \rightarrow \ell(B, \alpha, \beta) \sin _{n} \rightarrow \ell\left(v_{a}\right)$.

Proof: Let $1 / 2<E<2 / 3, \bar{b}(x)=\sum_{\ln _{n} 16 x^{E}} c_{n}(x) s_{n}$. And $\vec{t}(x)=\operatorname{ard}(2 \pi x) \sum_{\ln _{n} i x^{E}} \exp \left(-\frac{a^{2} n_{n}^{2}}{2 x}\right) s_{n}$ througtout the proof.

We firat show: if $s_{n}=O\left(n^{r}\right), r>0$, and $s_{n} \rightarrow \ell\left(v_{\alpha}\right)$ exen, $s_{n} \rightarrow(B, \alpha, \beta)$.

By the regularily of both nethodn 1 t 18 enough to prove the result for $l=0$. Horeoyer. by Leme (2.3) it guffices to show that $\bar{b}(x)=0(1) \omega x \rightarrow \bullet$.

2 kecall, froe keark (3.19), that for $n$ such that $1 l_{1} n f x^{5}$, ary 
lnteger $k>2$, and $x$ sufficiencly large

(3.22) $c_{n}(x)=a+(2 \pi x) \exp \left(-\frac{a^{2} n_{n}^{2}}{2 x}+8_{k}+k_{k}\right)$. where $g_{k}$ and $k_{k}$ are defined in Lema (3.8).

Choosing an 1 integer $k) \cdot 2 r+1$ (80 in particular $k 22$ ) we see inat for $x$ sufficlently large.

$$
\begin{aligned}
& b(x)-i(x)=\sum_{i n_{n} 16 x^{2}} s_{n}\left\{c_{n}(x)-\alpha \sqrt{ }(2 \pi x) \exp \left(-\frac{a^{2} n_{n}^{2}}{2 x}\right)\right\} \\
& -a /(2 \pi x) \sum_{\ln _{n} 16 x^{2}} s_{n} \exp \left(-\frac{a^{2} n_{n}^{2}}{2 x}\right)\left(\exp \left(s_{k}+R_{k}\right)-1\right) \\
& \alpha \sqrt{ }(2 \pi x) \sum_{\ln _{n} / 6 x^{6}} \sin _{n} \exp \left(-\frac{a^{2} n_{n}^{2}}{2 x}\right) \sum_{\mu=1}^{\infty} \frac{\left(B_{k}+R_{k}\right)^{\mu}}{\mu !} . \\
& =A_{1}(x)+A_{2}(x)+A_{3}(x),
\end{aligned}
$$

where

(3.23) $A_{1}(x)=\alpha \sqrt{ }(2 \pi x) \sum_{1 n_{n} 16 x^{5}} \sin _{n} \exp \left(-\frac{a^{2} n_{n}^{2}}{2 x}\right) \sum_{\mu=1}^{28} \frac{g_{k}^{\mu}}{\mu !}$.

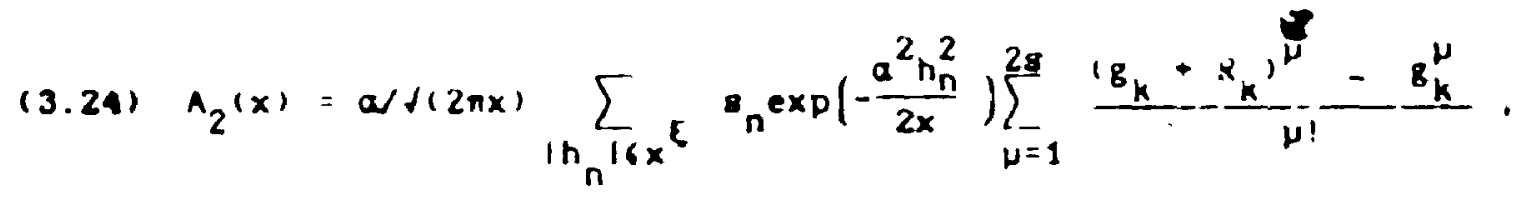

(3;25) $A_{3}(x)=a /(2 \pi x) \sum_{\ln _{n} 18 x^{c}} \sin _{n} \exp \left(-\frac{a^{2} n_{n}^{2}}{2 x}\right) \sum_{j=2 a+1}^{\infty} \frac{\left(g_{k}+k_{k}\right)^{\mu}}{\mu !}$. and the positite anteger b, r-1/2.

We procede to shou that $\left.A_{1}(x)=0(1), A_{2}(x)=0(1), A_{3}(x)=d 1\right)$, 
$8 \times \rightarrow \cdot$.

To see that $A_{1}(x)=0(1)$, consider for 16 w $2 \mathrm{~s}$

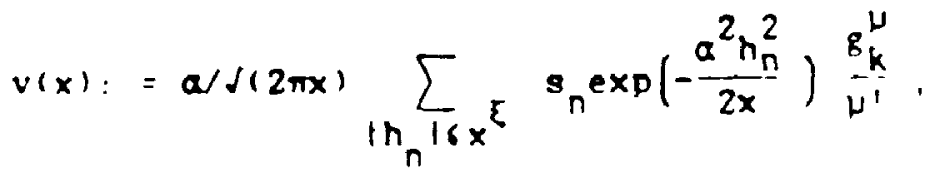

where $g_{k}^{\mu}$ is a finite sum of the form: $\sum_{1, j} \bar{b}_{1, j}, \frac{h_{n}^{j}}{x^{1}}$ and or fifu for

1

$1=\mu$. Or $j(1+\mu$ for $12 \mu+1$ (see Remark (3.20))

If we can shou that $v_{1,1}:=\bar{b}_{1,1} x^{-1 / 2} \sum_{\ln _{n} 16 x^{5}} s_{n} \exp \left(-\frac{a^{2} n_{n}^{2}}{2 x}\right) \frac{n_{n}^{1}}{x^{1}}$

is o(1) as $x \rightarrow$ - for those values of 1 and $f$ ment loned above, then

it follows that $v(x)=0(1)$ as $x \rightarrow \bullet$, and hence also that

$A_{1}(x)=0(1)$ \&s $x \rightarrow \bullet$.

To this end, the hypothesis $s_{n} \rightarrow$ orv $_{\alpha}$ ' implies that

$\sum_{k=0}^{\infty} s_{k} \exp \left\{-\frac{a(n-k)^{2}}{2 n}\right\}=o\left(n^{1 / 2}\right)$ as $n \rightarrow \infty, n=1,2, \ldots$

As $\left(8_{n}\right) 18$ of fintte order, Lemas (3.7) Iaplies that

$\sum_{n=0}^{\infty} 8_{n} \exp \left\{-\frac{a(n-x)^{2}}{2 x}\right\}=o\left(x^{1 / 2}, 88 x \rightarrow \cdots\right.$

Therefore, by Lenos (3.5) with $a=0$.

(3.26) $\sum_{n=0}^{\infty} \equiv_{n}(m-x)^{J} \exp \left\{-\frac{a(n-x)^{2}}{2 x}\right\}=0\left(x^{1 / 2(j+1)+n}\right)$ as $x \rightarrow \cdots$, 
for each integer jro, and each $\eta>0$.

Replacing $\times$ vith $x / a$ in $(3.26)$ ve obtaln.

(3.27) $\sum_{n=0}^{\infty} \sin _{n} h_{n}^{j} \exp \left(-\frac{a^{2} h_{n}^{2}}{2 x}\right)=o\left(x^{1 / 2(j+1)+\eta}\right)$ as $x \rightarrow \cdot$,

for each integer $\jmath$ so, and each $\eta>0$.

Since $\left.s_{n}=\operatorname{Or} n^{r}\right),(3.2)$ and $(3.3)$ of Leama (3.1), and (3.21) inply that,

(3.28) $\sum_{1 n_{n} 16 x^{c}} \sin _{n} n_{n}^{3} \exp \left(-\frac{a^{2} n_{n}^{2}}{2 x}\right)=o\left(x^{1 / 2(j+1)+\pi}\right)$ as $x \rightarrow \cdots$.

for each integer $\mathrm{j}>0$, and each $\eta>0$.

Consequent ly, by $(3.28$ )

$v_{1, j}=\bar{b}_{1, j} x^{-1 / 2-1} \sum_{\ln _{n} 16 x^{2}} \operatorname{son}_{n} n_{n}^{j} \exp \left(-\frac{a^{2} n_{n}^{2}}{2 x}\right)=0\left(x^{-1 / 2-1+1 / 2(j)+1)+n}\right)$

as $\times \rightarrow-$, for each $\eta>0$.

Now, for $1=\mu$, o6 $16 \mu$.

$$
\begin{aligned}
& \left.v_{1, j}=o\left(x^{-1+1 / 2} 1+\eta\right)=o\left(x^{-\mu+1 / 2}\right)^{j+\eta}\right)=o\left(x^{-\mu+1 / 2 \mu+\eta}\right) \\
& =o\left(x^{-1 / 2 \mu+\eta}\right)=o\left(x^{-1 / 2+\eta}\right), \mu>1 \\
& =0(1) \text { as } \times \rightarrow \bullet \text {, since } \eta>0 \text { can be chosen erbitrarily anall. }
\end{aligned}
$$

(e. $8 \cdot n=1 / 4)$ 
66

Next, for $1>\mu+1$, or $161+\mu$.

$v_{1, j}=o\left(x^{-1 / 2-1+1 / 2(j+1)+\eta}\right)=o\left(x^{-1 / 2+n}\right)$, since $12 j-\mu 2 j-1+1$, that is $-1+1 / 2(j+1) 60$.

Choosing $\eta=1 / 4$ we have: $\nabla_{1, j}=011$ as $\times \rightarrow \infty$.

Thus $v_{1, j}=0(1)$ ag $\times \rightarrow \oplus$, for all the appropriate values of 1 and 1 .

It follows that $A_{1}(x)=$ or $(1)$ as $x \rightarrow \cdots$.

In showing that $A_{2}(x)=0(1)$ as $x \rightarrow \infty$, observe that this will be done independently of the hypothesis $s_{n} \rightarrow o\left(v_{a}\right)$ : we only use $s_{n}=O\left(n^{r}\right.$ ).

since $s_{n}=O\left(n^{r}\right)$, it follows from (3.24) that,

$\left|A_{2}(x)\right| r \operatorname{LaJ} J(2 \pi x) \sum_{\ln { }_{n} \mid \delta x^{E}} n^{r} \exp \left(-\frac{\alpha^{2} h_{n}^{2}}{2 x}\right) \sum_{\mu=1}^{2 s}\left|\frac{\left(g_{k}+R_{k}\right)^{\mu}-g_{k}^{\mu}}{\mu^{\prime}}\right|$.

where $L$ is some positive constant.

Consider for $16 u \times 28$,

$u(x):=\frac{L a}{\mu ! \sqrt{(2 \pi x)}} \sum_{\ln { }_{n} / 6 x^{E}} n^{r} \exp \left(-\frac{a^{2} h_{n}^{2}}{2 x}\right)\left|\left(g_{k}+R_{k}\right)^{\mu}-g_{k}^{\prime \prime}\right|$.

If we can show that $u(x)=o(1)$ as $x \rightarrow \cdots$, then it follows that $A_{2}(x)=0(1)$ as $x \rightarrow$.

Hoy for $k>2,1 / 2<E<2 / 3, \ln n \mid 6 x^{5}, x \rightarrow \infty$, and by Lemma $(3.8)$,

$$
R_{k}=\left\{\frac{\left|h_{n}\right|^{k+1}+1}{x^{k}}\right\}=\text { or) and } g_{k}=0(1) \text {. }
$$

Hence for $j=1,2, \ldots, \nu$ 


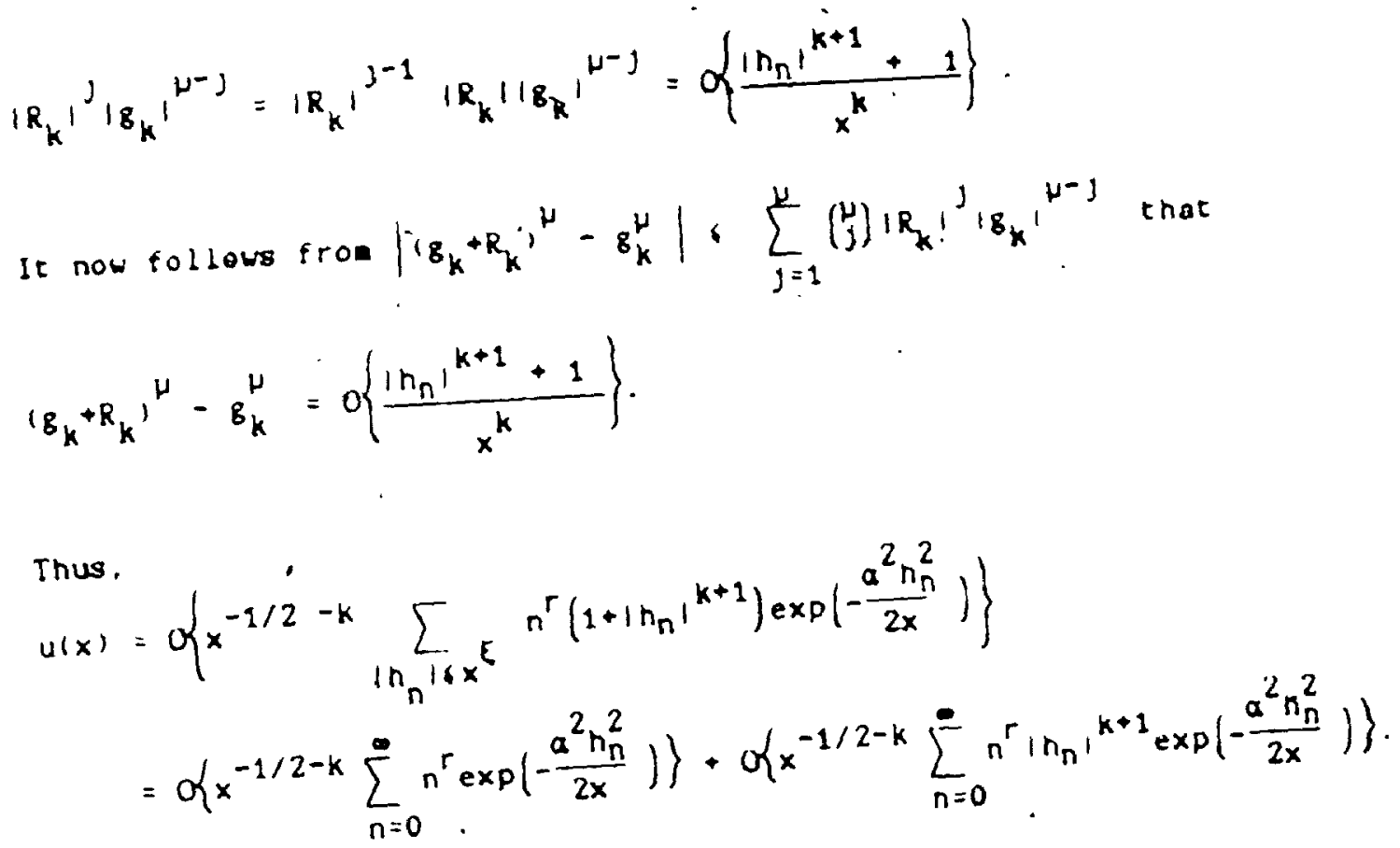

By Lema $(3.1)$,
$u(x)=O\left(x^{-1 / 2-k} x^{r+1 / 2}\right)+O\left(x^{-1 / 2-k} x^{r+1 / 2(k+1+1)}\right) 88 x \rightarrow \cdots$

$=0(1)$ as $x \rightarrow-$ since $k>2 r+1$.

It follaws that $A_{2}(x)=0(1)$ as $x \rightarrow \bullet$.

In showing that $A_{3}(x)=0(1)$ as $x \rightarrow-$ observe $(a s$ in the proof of: $A_{2}(x)=0(1)$ ss $x \rightarrow-$ that this will be done by only using the hypothesis $s_{n}=O\left(n^{r}\right)$.

Firat, for $1 / 2<k<2 / 3, \quad h_{n} 16 x^{k}, x \rightarrow-$, and by Lema $(3.8)$,

$$
\begin{aligned}
8_{k}+R_{k}=8_{2}+R_{2} & =\sum_{1=1}^{2} \sum_{j=0}^{1+1} b_{1, j} \frac{n_{n}^{j}}{x^{1}}+o\left\{\frac{\left|n_{n}\right|^{3}+1}{x^{2}}\right\} \\
& =o\left(\frac{1}{x}\right)+o\left(\frac{\left|n_{n}\right|}{x}\right\}+\left\{\frac{1}{2}\right\}+\left\{\frac{\left|n_{n}\right|}{x^{2}}\right\}+\left\{\frac{\left|n_{n}\right|^{2}}{x^{2}}\right\}
\end{aligned}
$$




$$
\begin{aligned}
& +\left\{\left\{\frac{\left|n_{n}\right|^{3}}{x^{2}}\right\}+o\left(\frac{1}{x}\right) \text {, s1nce } b_{1,2}=0\right. \\
& =0\left\{\frac{\left|n_{n}\right|+1}{x}\right\}+O\left\{\frac{\left|n_{n}\right|^{3}}{x^{2}}\right\} .
\end{aligned}
$$

since $\frac{1}{x^{2}}=O\left(\frac{1}{x}\right) \cdot \frac{\left|n_{n}\right|}{x^{2}}=O\left(\frac{\left|n_{n}\right|}{x}\right)$, and $\frac{\left|n_{n}\right|}{x}=O\left(x^{\xi-1}\right)=O(1)$

so that $\frac{\left|h_{n}\right|^{2}}{x^{2}}=O\left(\frac{\left|h_{n}\right|}{x}\right)$.

In particular, since $\frac{\left|n_{n}\right|}{x}=O\left(x^{E-1}\right)=O(1)$, and $\frac{\left|n_{n}\right|^{3}}{x^{2}}=O\left(x^{3 E-2}\right)=a(1)$ as $x \rightarrow-$. It follows that $g_{k}+R_{k}=o(1)$ as $x \rightarrow x^{x}$. CQnsequently. for $x$ sufficiently large: $\ln _{n} \mid 6 x^{\varepsilon}$, $1 / 2<k<2 / 3$, we obtain $1 g_{k}+R_{k} \mid$ \&cc1. Fron th1s we have:

$$
\sum_{\nu=28+1}^{\infty} \frac{18 k+R_{k}{ }^{\mu}}{\mu !}=O\left(18_{k}+\left.R_{k}\right|^{2 s+1}\right)
$$

By (3.25), the hypothesis $s_{n}=O\left(n^{r}\right)$, and the last estrate

$$
\text { (3.31) } \begin{aligned}
\wedge_{3}(x) & =\left\{x^{-1 / 2} \sum_{\ln n_{n} 16 x^{E}} n^{r} \exp \left(-\frac{a^{2} n_{n}^{2}}{2 x}\right) \sum_{\mu=28+1}^{-} \frac{\left|B_{k}+R_{k}\right|^{\mu}}{\mu !}\right\} \\
& =\left\{x^{-1 / 2} \sum_{\ln \sum_{n} 16 x^{\xi}} n^{r} \exp \left(-\frac{a^{2} n_{n}^{2}}{2 x}\right)\left|8_{k}+R_{k}\right|^{2 s+1}\right\}
\end{aligned}
$$

Next, it is legediate that for constants $a, b, c>0$, $(a+b+c)^{k}+3^{k}\left(a^{k}+b^{k}+c^{k}\right)$. So froe

$$
z_{k}+R_{k}=\left\{\frac{\left|n_{n}\right|+1}{x}\right\}+\mathcal{O}\left\{\frac{\left|n_{n}\right|}{x^{2}}\right\} \text { we obta1n: }
$$


69

$$
\begin{aligned}
\left|8_{k}+k_{k}\right|^{2 s+1} & \leqslant \frac{n}{x^{2 s+1}}\left\{1+\left|n_{n}\right|+\frac{\left|n_{n}\right|^{3}}{x}\right\}^{2 s+1} \\
& +\frac{3^{2 s+1} n}{x^{2 s+1}}\left\{1+\left|n_{n}\right|^{2 s+1}+\frac{\left|n_{n}\right|^{6 s+3}}{x^{2 s+1}}\right\} .
\end{aligned}
$$

where 1 is some constant.

Substitute ing the last inequality tor $\left|8_{k}+R_{k}\right|^{28+1}$ into $(3.31$ ) gives:

$$
\begin{aligned}
& \text { - (3.32) } A_{3}(x)=\left\{x^{-2 s-3 / 2} \sum_{\ln n_{n} 16 x^{k}} n^{r} \exp \left(-\frac{a^{2} n_{n}^{2}}{2 x}\right)\left(1+\left|n_{n}\right|^{2 s+1}+\frac{\left|n_{n}\right|^{6 s+3}}{x^{2 s+1}}\right)\right\} \\
& =\left\{x^{-2 s-3 / 2} \sum_{\ln \sum_{n} 16 x^{2}} n^{r} \exp \left(-\frac{\alpha^{2} n_{n}^{2}}{2 x}\right)\right\} \\
& \text { - }\left\{x^{-2 s-3 / 2} \sum_{\ln n \mid r x^{2}} n^{r}\left|n_{n}\right|^{2 s+1} \exp \left(-\frac{a^{2} n_{n}^{2}}{2 x}\right)\right\} \\
& +\left\{x^{-4 s-s / 2} \sum_{\ln n x^{1 / x^{5}}} n^{r}\left|n_{n}\right|^{6 s+3} \exp \left(-\frac{a^{2} n_{n}^{2}}{2 x}\right)\right\} \\
& =o\left(A_{3,1}(x)\right)+o\left(A_{3,2}(x)\right)+o\left(A_{3, j}(x)\right)
\end{aligned}
$$

Since we chose $8>r-1 / 2$ it follows that

(3.33) $\wedge_{3,1}(x)=O\left(x^{-2 s-3 / 2} x^{r+1 / 2}\right)=o(1)$ as $x \rightarrow \cdots$. by Lem (3.1) with $p=0$.

(3.34) $A_{3,2}(x)=0\left(x^{-2 s-3 / 2} x^{r+1 / 2(28+1+1)}\right)=0(1), x \rightarrow \bullet$, by Lena (3.1) with $p=20+1$. 
(3.35) $A_{3,3}(x)=O\left(x^{-4 s-5 / 2} x^{r+1 / 2(6 s+3+1)}\right)=0(1)$ as $x \rightarrow \bullet$

by Leme (3.1) with $p=68+3$.

Subet 1tut ing $(3.33),(3.34),(3.35)$ in $(3.32)$ ve have, as required, $A_{3}(x)=0(1)$ 28 $x \rightarrow \cdots$.

Therefore, we have shown that

(3.36) $\bar{b}(x)-\bar{E}(x)=A_{1}(x)+A_{2}(x)+A_{3}(x)=0(1)$ as $x \rightarrow-$.

Next, since $s_{n} \rightarrow O\left(v_{\alpha}\right)$, it follows by Leana (3.7) that

(3.37) $\left[\frac{a}{2 \pi x}\right)^{1 / 2} \cdot \sum_{n=0}^{\infty} s_{n} \exp \left\{-\frac{\alpha(n-x)^{2}}{2 x}\right\}=0(1)$ as $x \rightarrow \cdots$

Replacing $x$ by $x / \alpha$ in $(3.37)$ ve have:

(3.38) $\quad \alpha \delta(2 \pi x) \sum_{n=0}^{\infty} s_{n} \exp \left(-\frac{a^{2} h_{n}^{2}}{2 x}\right)=o(1)$ as $x \rightarrow \cdots$

Recalling that $s_{n}=O\left(n^{r}\right),(3.38)$, and $(3.2),(3.3)$ of Leana $(3.1)$

with $p=0,10 p l y$ that

(3.39). $\bar{t}(x)=0(1)$ as $x \rightarrow$.

Therefore, frow (3.36) and (3.39) it. follows that $\bar{b}(x)=0(1) x \rightarrow-$

Th1s conpletes the first part of the theoren.

Now show: if $g_{n}=O\left(n^{r}\right), r>0$, and $a_{n} \rightarrow \ell(B, \alpha ; \beta)$, then $g_{n} \rightarrow \ell\left(v_{\alpha}\right)$.

Again we assume $l=0$. By Reanrk (3.19), Lewas (2.3), and our hypothesis, 


$$
=\left\{x^{-1 / 2} \sum_{n=0}^{\infty} n^{r} \exp \left(-\frac{a^{2} n_{n}^{2}}{2 x}\right)\right\}=O\left(x^{r}\right)
$$

Let $r:=\operatorname{lnf}\left\{\bar{r}: \bar{E}(x)=O\left(x^{\gamma}\right)\right\}$. It follows fron (3.41) that rrt. If $r<0$. then $\bar{t}(x)=0(1)$ as $x \rightarrow-$. uhich 18 what we eventually want to show. Therefore let us assume that $0<y, r$, and prove that $\bar{t}(x)=0(1)$ as $x \rightarrow \cdots$.

Fron the definition of $y$ we have: $\bar{t}(x)=o\left(x^{\gamma+\varepsilon}\right.$, as $x \rightarrow-$ for any $\varepsilon>0$, and that $\bar{E}(x)=o\left(x^{y-\delta}\right)$ as $x \rightarrow$ - for no $\delta>0$.

\section{Consider,}

$A_{1}(x)=\alpha / s(2 \pi x) \sum_{h_{n} 16 x^{E}} s_{n} \exp \left(-\frac{a^{2} n_{n}^{2}}{2 x}\right) \sum_{\mu=1}^{2 g} \frac{8_{k}^{\mu}}{\mu !}$, and for $16 \mu<2 s$ let $p(x)=\alpha / 2 \pi x) \sum_{\ln { }_{n} 16 x^{5}} s_{n} \exp \left(-\frac{a^{2} h_{n}^{2}}{2 x}\right) \frac{g_{k}^{\mu}}{\mu !}$, where of is a tinile sue of the form $\sum_{1, j} \bar{b}_{1, j} \frac{n_{n}^{j}}{x^{1}}$, or $j 6 \mu$ for $1=\mu$, or $j 61+\mu$ for $12 \mu+1$. (see Reark $(3.20))$

Hext, let

$p_{1, j}=\bar{b}_{1, j} x^{-1 / 2} \sum_{\ln n} 16 x^{E} g_{n} \exp \left(-\frac{a^{2} n_{n}^{2}}{2 x}\right) \frac{n_{n}^{J}}{x^{1}}$ for those values of 1

and $\mathrm{J}$ eent loned above.

Since $\bar{t}(x)=0\left(x^{\gamma+\epsilon}\right.$, as $x \rightarrow-$, for any $\varepsilon>0$, it followe that

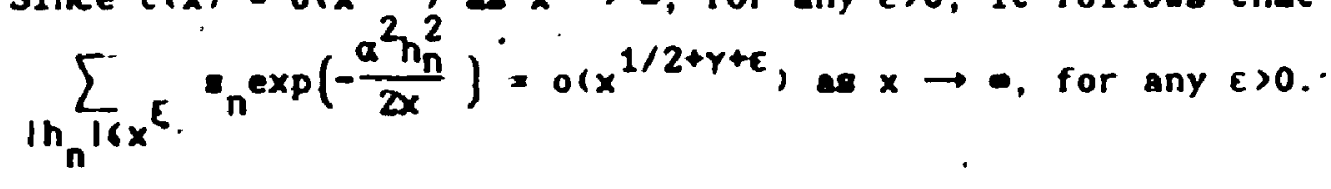




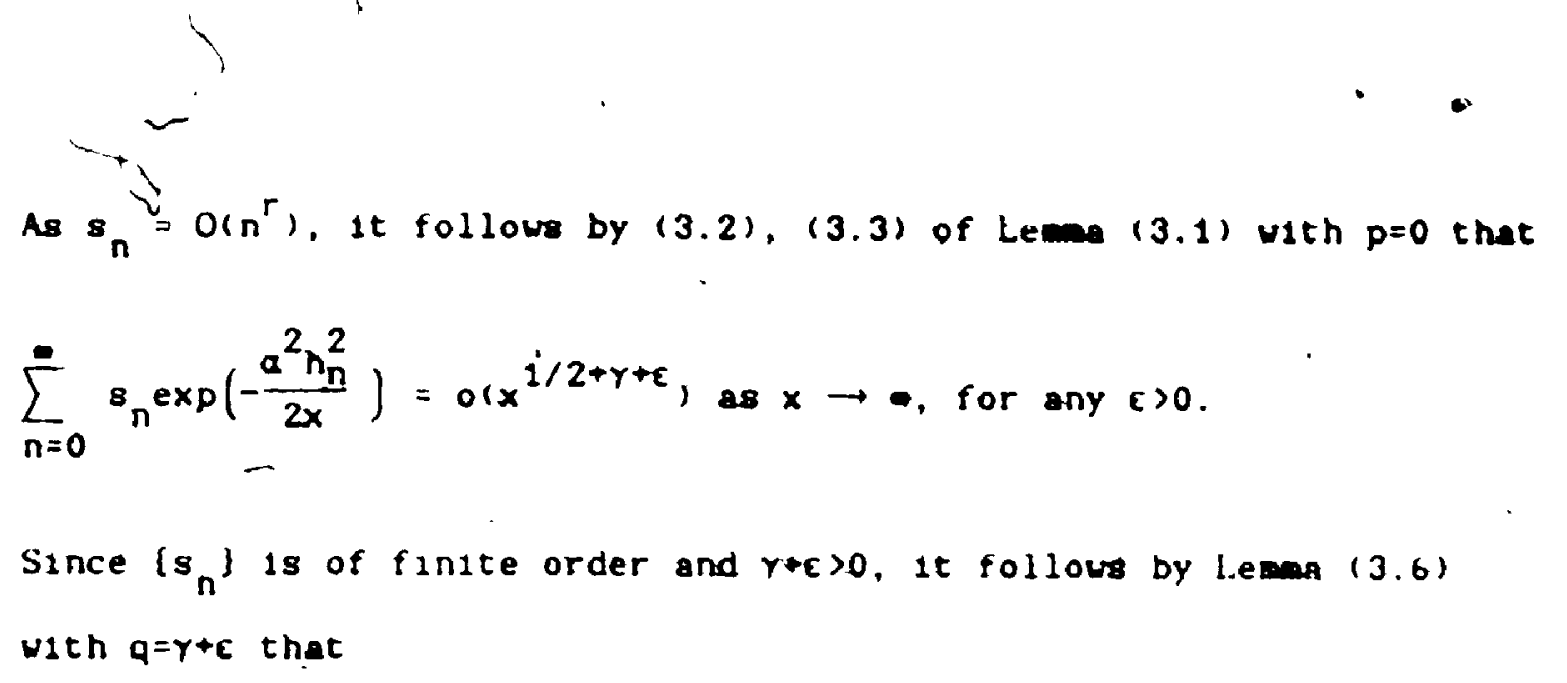

(3.12) $\sum_{n=0}^{\infty} s_{n} n_{n}^{j} \exp \left(-\frac{a^{2} n_{n}^{2}}{2 x}\right)=o\left(x^{1 / 2(j+1)+\gamma+\varepsilon+n}\right)$

as $x \rightarrow-$, for each integer $j>0$ and each $\eta>0$.

Since $s_{n}=O\left(n^{r}\right),(3.2)$ and $(3.3)$ of Leme $(3.1)$, and $(3.42)$ laply that

$\sum_{1 n_{n} 16 x^{\varepsilon}} s_{n} n_{n}^{j} \exp \left(-\frac{a^{2} n_{n}^{2}}{2 x}\right)=o\left(x^{1 / 2(j+1)+y+\varepsilon+n}\right)$

as $x \rightarrow-$, for each integer $1>0$ and each $n>0$

Consequent $I y, p_{1, j}=0\left(x^{-1 / 2-1+1 / 2(j+1)+y+\varepsilon+\eta}\right)$ as $x \rightarrow \cdots$. where $E>0$ and $n>0$ are both arbitrary.

Nov for $1=\mu$, or $j 6 \mu$,

$p_{t, j}=0\left(x^{-1 / 2-\mu+1 / 2(j+1)+y+c+\eta}\right)$

$=0\left(x^{-\mu+1 / 2 \mu+\gamma+\varepsilon+\eta}\right)$, or $j 6 \mu$

$$
\left.=0\left(x^{-1 / 2+y+c+n}\right), \quad \mu\right) 1 \text {. }
$$

Choosing $E>0$ and $n>0$ sadl enough we have: $p_{1, j}=0\left(x^{y-\delta} 0\right.$, for $\delta_{0}>0$. (e:s. for $c=n=1 / 8$, then $\left.\delta_{0}=1 / 4\right)$ 
In the second case: $12 \mu+1$, or $j 61+\mu$,

$$
\begin{aligned}
& p_{1, j}=o\left(x^{-1 / 2-1+1 / 2(j+1)+y+c+n}\right) \\
& =o\left(x^{-1 / 2-1 / 21+1 / 2(j-1+1)+y+\varepsilon+\eta}\right) \\
& =o\left(x^{-1 / 2-1 / 2(\mu+1)+1 / 2(\mu+1)+\gamma+\varepsilon+\eta}\right), 1 \geqslant \mu+1, j-16 \nu \\
& =0\left(x^{-1 / 2+\gamma+c+n}\right) \\
& =0\left(x^{Y-\delta_{0}}\right) \text { with the same } \delta_{0}>0 \text { as above. }
\end{aligned}
$$

Thus, for the approprtate values of 1 and $1, p_{1, j}=x\left(x^{\gamma-\delta_{0}}\right)$ for a

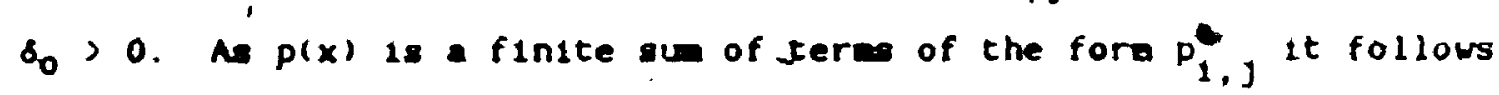
that $p(x)=o\left(x^{y-\delta_{0}}\right)$ for $a \delta_{0}>0$. A sxellar reason shows that $A_{1}(x)=0\left(x^{\gamma-\delta_{0}}\right)$ for $\left.\delta_{0}\right) 0$. It follow from $\vec{t}(x)+A_{1}(x)=0(1)$, and $A_{1}(x)=o\left(x^{\gamma-\delta_{0}}\right)$ that $\bar{t}(x)=o\left(x^{\gamma-\delta_{0}}\right)+o(1)$ for a $\delta_{0}>0$. Now, if $y-\delta_{0}, 0$, then $\vec{t}(x)=0\left(x^{\gamma-\delta_{0}}\right)$ for $, \delta_{0}, 0$, which contradicts the alniality of $r$. Thu, $r-\delta_{0}, 0$ and this inplies that $\bar{E}(x)=o(1)$ es $x \rightarrow$.

Finally, ance $*_{n}=O\left(n^{r}\right),(3.2),(3.3)$ of Leman $(3.1)$ and $E(x)=o(1)$ as $x \rightarrow \infty, 1$ ieply that

$a \sqrt{ }(2 \pi x) \sum_{n=0}^{\infty} a_{n} \exp \left(-\frac{a^{2} n_{n}^{2}}{2 x}\right)=0(1) \propto x \rightarrow \cdots$

Replecing $x$ with ox we have: 
$\left(\frac{a}{2 \pi x}\right)^{1 / 2} \sum_{n=0}^{a} s_{n} \exp \left(-\frac{a(n-x)^{2}}{2 x}\right)=0(1)$ as $x \rightarrow$, and of course.

$\left(\frac{a}{2 \pi n}\right)^{1 / 2} \sum_{n=0}^{\infty} s_{n} \exp \left(-\frac{\alpha(n-n)^{2}}{2 m}\right)=0(1)$ as $m \rightarrow \rightarrow, n=1,2, \ldots$

1.e. $s_{n} \rightarrow 0\left(v_{a}\right) \cdot 1 / 1$

The next theorea 13 an equiconvergence result applicable to is ${ }_{n}$, for uhich neither convergence statement $s_{n} \rightarrow \ell\left(V_{\alpha}\right), s_{n} \rightarrow\{(B, a, \beta)$ need hold. Theorem (3.21) 13 a more general theorem than (3.43) but less general in that the equiconvergence property is lost.

(3.43) Theoree (see Borwein and Robinson [7], Theorem 2): I.f $s_{n}=0\left(n^{r}\right), r<1 / 2$, then

$\sum_{n=N_{0}}^{\infty} s_{n}\left\{c_{n}(x)-a / d(2 \pi x) \exp \left(-\frac{a^{2} h_{n}^{2}}{2 x}\right)\right\}=0(1) \operatorname{ss} x \rightarrow a$

- In particular $s_{n} \rightarrow \ell(B, a, B) \infty_{n} \rightarrow l\left(v_{\alpha}\right)$

Proaf: Let $a_{n}(x):=c_{n}(x)-a / d(2 \pi x) \exp \left(\frac{a^{2} h_{h}^{2}}{2 x}\right)$ for $n 2 N_{0}$, and $1 / 2<E<2 / 3$.

wite,

$$
\begin{aligned}
& \text { (3.44) } v(x):=1 \sum_{n=N_{0}}^{2} s_{n} a(x) 1
\end{aligned}
$$

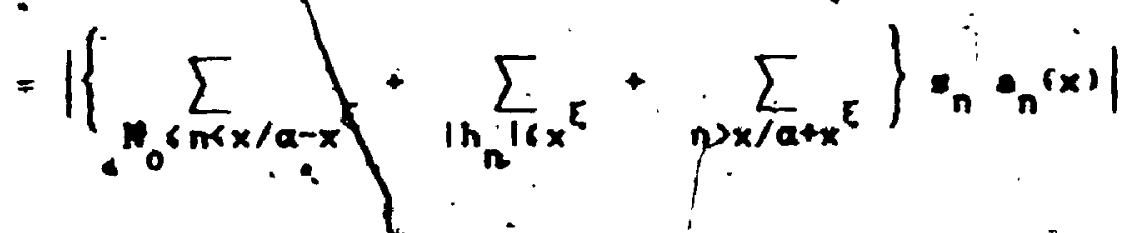

$$
\begin{aligned}
& \text { c. } v_{1}(x)+v_{2}(x)+v_{8}(x)
\end{aligned}
$$


76

vhere.

$$
\begin{aligned}
& v_{Y}(x)=\left|\sum_{n_{0} \sigma n x / a-x^{E}} n_{n} e_{n}(x)\right|, v_{2}(x)=\left|\sum_{\left|n_{n}\right| \sigma x^{\varepsilon}} s_{n} \cdot n(x)\right| \text {. and } \\
& v_{3}(x) \cdot \sum_{n>x / a+x^{E}} s_{n} n_{n}(x) \mid
\end{aligned}
$$

(3.45) $\left.v_{1}(x)=\left\{\sum_{N_{0}\left(n<x / a-x^{\varepsilon}\right.} n^{r} c_{n}(x)\right\}+\sigma x^{-1 / 2} \sum_{N_{0}\left[n<x / a-x^{\xi}\right.} n^{r} \exp \left(-\frac{a^{2} n_{n}^{2}}{2 x}\right)\right\}$

$$
=0(1) \mathrm{ns} x \rightarrow 3 \text {, by Lezmen (2.3), and (3.3) of }
$$

Lemes 13.11 vith $p=0$.

- (3.4) $v_{3}(x)=\left\{\sum_{n>x / \alpha+x} n^{r} c_{n}(x)\right\}+\sigma\left\{x^{-1 / 2} \sum_{n>x / \alpha+x} n^{r} \exp \left(-\frac{a^{2} n_{n}^{2}}{2 x}.\right)\right\}$

$=$ o(1) as $x \rightarrow$. by Lemen (2.3). and (3.2) of

Lemo 19.11 oleh $p=0$.

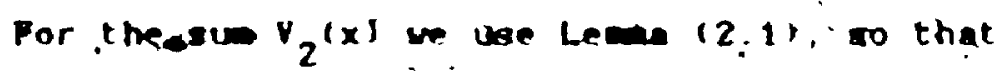

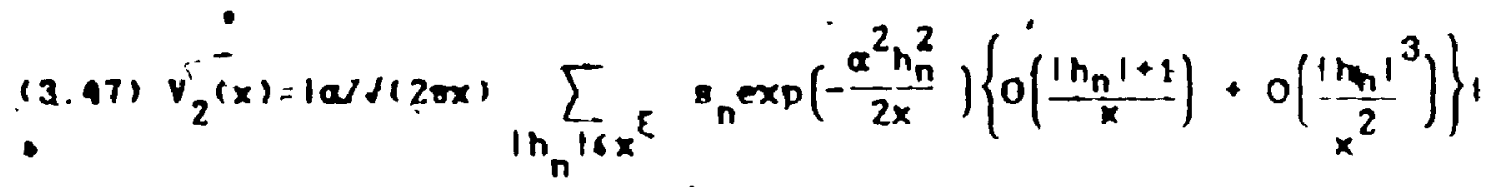

$$
\begin{aligned}
& =\left\{x^{-1 / 2} \sum_{\ln _{n} \mid 6 x^{E}} n^{r} \exp \left\{-\frac{a^{2} n_{n}^{2}}{2 x} j\left(\frac{\left|n_{n}\right|+1}{x}+\frac{\left|n_{n}\right|^{3}}{x^{2}}\right)\right\} .\right. \\
& =\left\{x^{-3 / 2} \sum_{1 n_{n}^{2}+\left(x^{2}\right.} n^{r} d n_{n} i \exp \left(-\frac{a^{2} n_{n}^{2}}{2 x}\right)\right\}
\end{aligned}
$$




$$
\begin{aligned}
& +\left\{x^{-3 / 2} \sum_{\ln n x^{16}} n^{r} \exp \left(-\frac{\alpha^{2} n_{n}^{2}}{2 x}\right)\right\}
\end{aligned}
$$

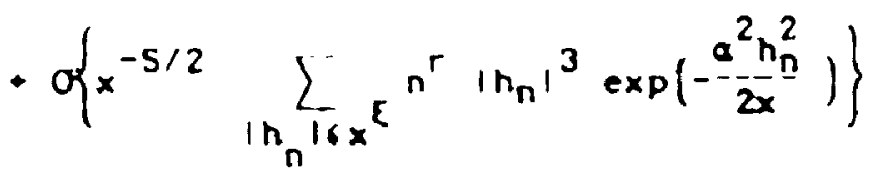

$$
\begin{aligned}
& =o\left(v_{2,1}(x)\right) \cdot O\left(v_{2,2}(x)\right) \cdot O\left(v_{2,3}(x)\right)
\end{aligned}
$$

Since $r<1 / 2$,

$v_{2,1}(x)=O\left(x^{-3 / 2} x^{r+1 / 2(1+1)}\right)=O\left(x^{r-1 / 2}\right)=O(1)$ es $x \rightarrow \cdots$,

by Lame (3.1) with $p=1$.

$v_{2,2}(x)=O\left(x^{-3 / 2} x^{r+1 / 2}\right)=O\left(x^{r-1}\right)=o(1)$ es $x \rightarrow-$

by l.emo (3.1) with $p=0$.

$v_{2,3}(x)=O\left(x^{-5 / 2} x^{r+1 / 2(3+1)}\right)=O\left(x^{r-1 / 2}\right)=0(1)$ as $x . \cdots$

by Leina (3.1) with $p=3$.

It follows "froe (3.47) that

(3.48) $v_{2}(x)=o(1)$ as $x \rightarrow \infty$

Subst 1tut ing (3.45), (3.48), and (3.46) in (3.44) yields the required resule.

For the finel pert, assue firgt, that $g_{n} \rightarrow$ (B.a.B),

1.e. $\sum_{n=N_{0}}^{\infty} s_{n} c_{n}(x) \rightarrow l$ is $x \rightarrow \infty$. Then es $x \rightarrow \infty$.

$\alpha / J(2 \pi x) \sum_{n=N_{0}}^{\infty} B_{n} \exp \left(-\frac{\alpha^{2} n_{n}^{2}}{2 x}\right)=\sum_{n=K_{0}}^{\infty} \otimes_{n} c_{n}(x)-\sum_{n=N_{0}}^{\infty} x_{n} a_{n}(x) \cdot \rightarrow l$.

Replacing $\times$ with ax we have: 
$\left(\frac{a}{2 \pi x}\right)^{1 / 2} \sum_{n=k_{0}}^{\infty} 8_{n} \exp \left\{-\frac{\alpha(n-x)^{2}}{2 x}\right\} \rightarrow 2$ es $x \rightarrow \cdots$

Since the finite $8=\left(\frac{a}{2 \pi x}\right)^{1 / 2} \sum_{n=0}^{N_{0}-1} s_{n} \exp \left\{-\frac{a(n-x)^{2}}{2 x}\right\}=0(1)$

we have: $\left(\frac{a}{2 \pi x}\right)^{1 / 2} \sum_{n=0}^{e} s_{n} \exp \left\{-\frac{a(n-x)^{2}}{2 x}\right\} \rightarrow \ell$ es $x \rightarrow$. and of

course, $\left(\frac{a}{2 m}\right)^{1 / 2} \sum_{n=0}^{\infty} s_{n} \exp \left\{-\frac{a(n-a)^{2}}{2 a}\right\} \rightarrow l a s \rightarrow \rightarrow-\infty=1,2 \ldots$

1.e. $x_{n} \rightarrow\left(v_{\alpha}\right)$.

Conversely, we any assune by regularity that $l=0$, so that

$a_{n} \rightarrow O\left(v_{\alpha}\right.$ ' 18 our hypotpesis and we vant to shou that $s_{n} \rightarrow O(B, a, \beta)$.

Since $\left(\frac{\cdot a}{2 \pi n}\right)^{1 / 2} \sum_{k=0}^{\infty} y_{k} \exp \left\{-\frac{a(n-k)^{2}}{2 n}\right\}=0(1)$ es $n \rightarrow-, n-1.2 \ldots$

and $\left[\mathrm{s}_{n}\right]$ ta of finite order, Iese (3.7) inplies that

$\left(\frac{a}{2 \pi x}\right)^{1 / 2} \sum_{n=0}^{\infty} e_{n} \exp \left\{-\frac{g(n-x)^{2}}{2 x}\right\}=\dot{0}(1)=2 x \rightarrow \cdots$

Hence, $\alpha d(2 \pi x) \sum_{n=0}^{-}{ }_{n} \exp \left(-\frac{a^{2} n_{n}^{2}}{2 x}\right)=$ o(1), which implies that

$\alpha J(2 \pi x) \sum_{n=N_{0}}^{\infty} e_{n} \exp \left(-\frac{a^{2} n_{n}^{2}}{2 x}\right)=01,11 \ldots x \rightarrow \cdots$

Thue, $\infty \times \rightarrow$.

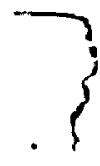

$\sum_{n=N_{0}}^{\infty} e_{n} \dot{c}_{n}(x)=\sum_{n=N_{0}}^{\infty} g_{n} \cdot n_{n}(x)+\operatorname{ard}(2 \pi x) \sum_{n=N_{0}}^{\infty} e_{n} \exp \left(-\frac{a^{2} n_{n}^{2}}{2 x}\right)=0(1)$. 
1.e. $s_{n} \rightarrow O(B, a, \beta)$.

The next four results are due to Falhaber $(10)$ and Bingina (2)

and are stated as lemas. Thege w1ll be uged with Theorea $(3.21)$ to obtain equivalences between the $(B, a, B\rangle$ nethod and those of Euler,. Meyer-Konig, and Taylor, under the assuaption $\left(8_{n}\right) 18$ of findte order. Later we obtain anclusions under the veaker assuaption ITr.'

(3.49) Lemar $(2)$, coroliary 3$)$ : For $\left(x_{n}\right)$ of finite order. $a_{n} \rightarrow l(B) \sin _{n} \rightarrow \ell\left(v_{1}\right)$

This 18, of course, special case of Theorea (3.21) with $a=8=1$.

(3.50) Lema( (2), corollary 4): For $0<a<1$, and $\left(a_{n}\right)$ of fintte order,

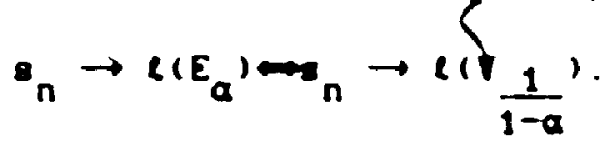

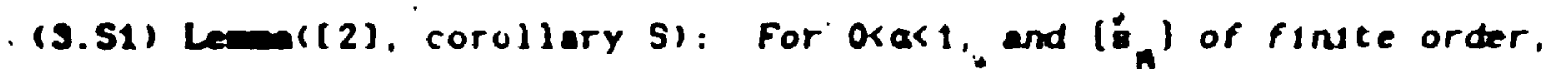

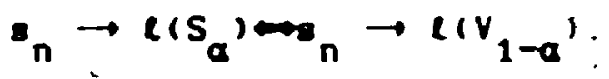

(3.52) Lema ( $\{2)$ " corollary 6): For o<a<1, and $\left(8_{n}\right)$ of finite order. $a_{n} \rightarrow \ell\left(T_{a}\right) e_{n} \rightarrow l\left(v_{\frac{1}{a}-1}\right)$

Lema (3.53) is alyo due to Binghan (2) but has also been proved earlier by Borvein and Robineon( $(7)$, Theoree 3$)$ under the condition $e_{n}=o(J n)$. 
(3.53) Leem( $[2]$. Theorea 3): If $0<a<a^{\prime}<0$ and $\left(g_{n}\right)$ is of finitie order, then $s_{n} \rightarrow \ell\left(V_{a}{ }^{\prime-\infty}{ }_{n} \rightarrow l\left(V_{a}\right)\right.$

Note: The proof of Lema (3.53) 18 obtained by using some known resultg linking the circle wethods of convergence. But, we can get the sase inclusion under the gave hypotheges by using Theorea (3.21) and a result due to Borwein ( see Result(I) in Reark (3.58), as follows:

$8_{n} \rightarrow l\left(V_{\alpha^{\prime}}\right){ }_{n} \rightarrow \ell\left(B, \alpha^{\prime}, B\right)+\alpha_{n} \rightarrow l(B, \alpha, B)+B_{n} \rightarrow l\left(V_{\alpha}\right)$

(3.54) Corollary: for $0<a<1$, and $\left(\mathrm{s}_{n}\right)$ of finste order. $e_{n} \rightarrow l\left(E_{a}\right) \rightarrow B_{n} \rightarrow l\left(B, \frac{1}{1-a}, B\right)$.

Proof: By Lema (3.50) and Theorea (3.21) we have: ${ }_{n} \rightarrow \ell\left(E_{\alpha}\right) \infty_{n} \rightarrow \ell\left(\frac{1}{1-a}\right) \infty_{n} \rightarrow \ell\left(B \cdot \frac{1}{1-a} \cdot \beta\right) . / 1 /$

(3.5s) Corollary: For ocas 1 , and $\left[a_{n}\right]$ of finlte order. $n_{n} \rightarrow \ell\left(S_{\alpha}\right) \infty n \rightarrow \ell(\beta, 1-\alpha, \beta)$.

Proof: By Lewa ( $\{.51$ ) and Theoren (3.21) we obtain:

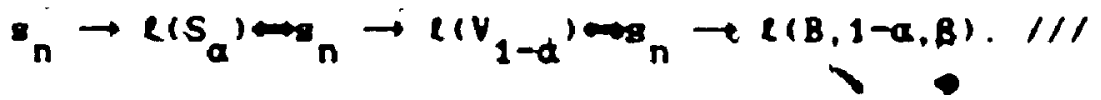

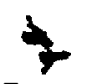

(S.S6) Corollary: For $\operatorname{Qxa} 1$, and $\left(8_{n}\right)$ of finite order. $a_{n} \rightarrow\left(T_{a}\right)-e_{n} \rightarrow \ell\left(B, \frac{1}{\alpha}-1, \beta\right)$

Proof: By Leme (3.52) and Theoree (3.21) we have: 
$8_{n} \rightarrow \ell\left(T_{\alpha}\right) \log _{n} \rightarrow \ell\left(V_{\frac{1}{a}}-1\right) \log _{n} \rightarrow \ell\left(B, \frac{1}{a}-1, \beta\right) .1 /$

(3.57) Corollary: For orar $a^{\prime}<0$, and $\left[s_{n}\right\}$ of fimte order.

$s_{n} \rightarrow \ell(B, \alpha \geq \beta) \theta_{n} \rightarrow \ell(B, \alpha, \beta)$.

Proof: By applying theores (3.21) tulce, and Leme (3.53) we

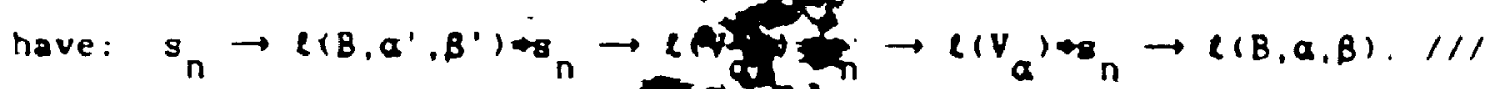

(3.58) Reaurk: Borvein((5), Result (I)) has proved the following result :

(I) If $a^{\prime}>a>0$ and, for any non-negative integer $M>-\beta / \alpha$,

$\sum_{n=n}^{\infty} \frac{a_{n}}{r\left(a_{n}+\beta\right)} x^{n}$ is convergent for all $\times>0$, then

$\sum_{n=0}^{\infty} a_{n}=\left(\beta, \alpha^{\prime}, \beta^{\prime}\right) \cdot \sum_{n=0}^{\infty} a_{n}=\ell(\beta, \alpha, \beta)$.

(i) 18 stranser than Corollary (3.57) in that for $\left(8{ }_{n}\right)$ of findte order (1.e. $s_{n}=0\left(n^{r}\right)$ for sose $\left.r\right)$, it then followe that

$\sum_{n=k}^{\infty} \frac{a_{n}}{\Gamma(\operatorname{con}+\beta)} x^{n} 18$ convergent for $11 \times>0$, where $e_{n}=g_{n} ! s_{n-1}$.

$a_{10}-0$. This can shown as followa since $s_{n}=0\left(n^{r}\right)$, then also $a_{n}=\operatorname{oin}{ }^{r}$, , 1.e. $\mathrm{la}_{\mathrm{n}}, \cdot \mathrm{Kn}^{\mathrm{r}}$ for some $\mathrm{x}>0$. Therefore.

$\frac{\operatorname{len} !^{\prime}}{\Gamma(a n+\beta)} x^{n}, \frac{x_{n}^{r}}{\Gamma(\alpha+\beta)} x^{n}, n$ on. Unins the version of Stirline:

formula given in the proof of Leme 12.21, we see that 

Borvein's Regult (I).

(3.60) Corollary: Suppose that $a>1$, and that $\left(T_{r}\right.$ ) holds. Then $s_{n} \rightarrow \ell(B, a, B) 1$ polles $s_{n} \rightarrow \ell\left(E_{1-\frac{1}{a}}\right)$.

Proof: As in the last proof, our hypotheses imply that $s_{n}=O\left(n^{r}\right)$. By Theored $(3.21), s_{n} \rightarrow \ell\left(v_{\alpha}\right)$. Since $\left(s_{n}\right)$ is of finite $\infty$ order we have by Lema $(3.50), 8_{n} \rightarrow \ell\left(E_{1-\frac{1}{a}}\right.$. Note for $\left.a\right) 1$.

$0<1-\frac{1}{a}<1$, which is required in the definition of the $\mathrm{A}-\frac{1}{\bar{a}}, 111$

(3.61) Corollary: Suppose that $0<a<1$, and $\left(T_{r}\right)$ holds. Then $s_{n} \rightarrow(B, a, B)$ loplies $\theta_{n} \rightarrow$ ers $_{1-\alpha}$.

Proof: As in the previaus proof our hypotheses inply that $s_{n}=O\left(n^{r}\right)$, and hence $s_{n} \rightarrow \ell\left(v_{a}\right)$. Since $\left(s_{n}\right)$ is of finite order, $":$ Lemar $(3.51)$ implies that $x_{n} \rightarrow e\left(s_{1-a}\right)$. Note for $0<a<1$. $0<y-a<1$ which 18 refaired in the definition of the $s_{1-a}$ method. 111

(3.62) Corollary: Suppose that $\left(T_{r}\right)$ holds and that $B_{n} \rightarrow \ell(B, a, B)$. Then $s_{n} \rightarrow l\left(T_{1 / 1+a)}\right)$.

Proof: Thi hyjotheses imply that $\left(g_{n}\right)$ is of ginite order, and hence that $s_{n} \rightarrow \ell\left(\nabla_{\alpha}\right)$. By Lewa $(3.52), s_{n} \rightarrow l\left(T_{1 /(1+a)}\right)$. Hote $\alpha>0$ is assuned in the defidition of the $(B, a, B)$ athod and this 

$\boldsymbol{\theta}$

$i^{0}$

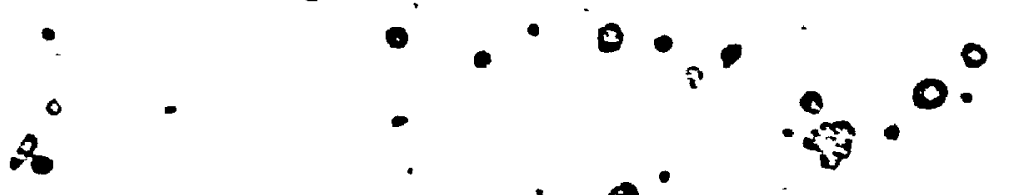

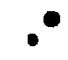

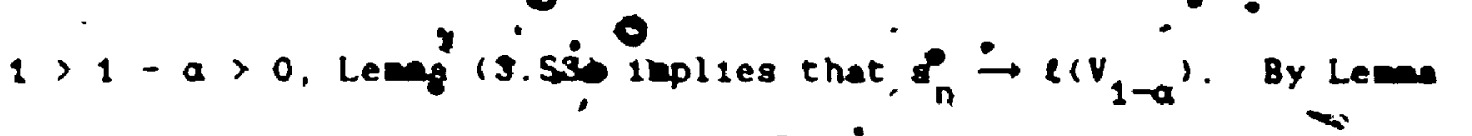
$(3.51), s_{n} \rightarrow e\left(S_{a}\right) i / 1 /$

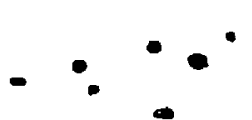

(3.66) Corollary: Suppose that ( $T_{0}$ ) holds and that $8_{n} \rightarrow l\left(S_{a}\right)$. Then $s_{n} \rightarrow(x)$.

Proof: The hypothesis $s_{n} \rightarrow l{ }^{\prime} S_{a}^{\prime}$ inplies that

$s_{a}(a):=(1-a)+1 \sum_{1=0}^{\infty}\left(\begin{array}{c}m+1 \\ 1\end{array}\right) a^{1} s_{1}=0(1)$, and nẹce $s_{n}=0(1)$, by

Sitaraman (c18). Theorea 1 with $r=0)$. By pesult due to heyer-könig

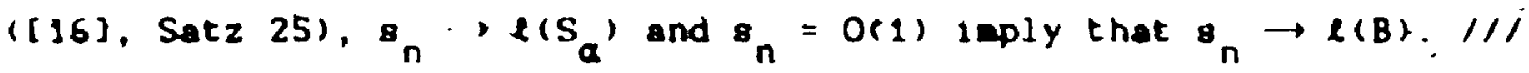

Colbining the last two regults we have:

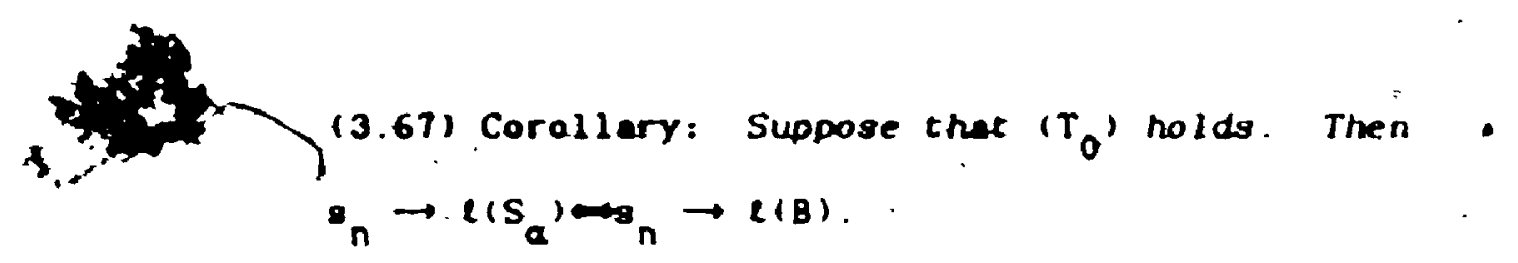

1 


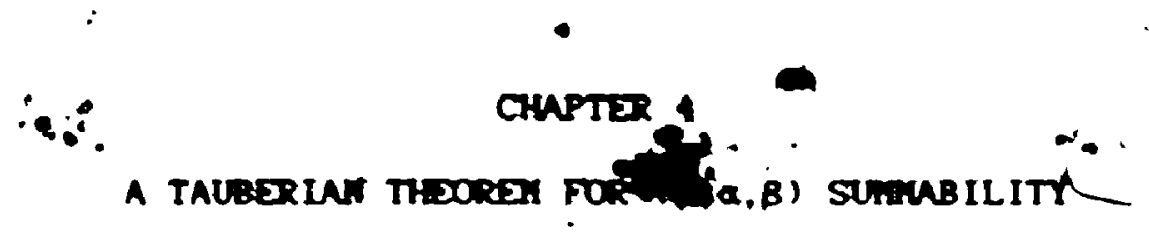

In this chapter ve prove our main result, Theored (4.2). This result will be deduced from a corresponding theorem for $s_{a}$ sumability ; due to sitaraman.

(4.1) Lema (S1taraman $[18)$. Theoren 2): If ( $T_{r}$ ) holds and $s_{n} \rightarrow \ell\left(s_{a}\right)$, then $s_{n} \rightarrow \ell\left(C_{2 r}\right)$.

Note the corresponding Tauberion theorem for Borel sumability is due to Rajagopal ( 1171 . Theorea 2$)$, and can be deduced by applying Corollary (3.65) and Sitaraman's Theurem.

(4.2.) Theorea: If $\left(T_{r}\right.$ ) holds and, $s_{n} \rightarrow l(B, a, B)$, then $s_{\text {A }} \rightarrow e\left(C_{2 r}\right)$.

Proof: It is enough to prove the theoren for $1>a>0$. That 1s, 1t suffices to show: $\left(T_{r}\right)$ and $s_{n} \rightarrow l\left(B_{r} a^{\prime}, B\right) 1$ aply $s_{n} \rightarrow l\left(C_{2 r}\right)$, where $1>a^{\prime}>0$. For suppose $a, 1>a^{\prime}>0$. By Corollary (3.59) the hypotheses w11l imply s $\rightarrow l\left(B, a^{\prime}, B\right)$. Thus the result for all a $>$ follows froe the corresponding result vith 1 , $a>0$. Restrictio to 1 , a >0, Corollary i3.61) Implies ${ }_{n} \rightarrow \ell\left(S_{1-a}\right.$.

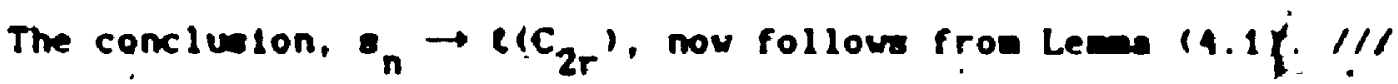

(4.3) benems A elighely different proof of Theoren 14.21 is as 
follow: By Theorea (2.15) we have $s_{n}=O\left(n^{r}\right.$, , and it follow by Theorem (3.2i) that $s_{n} \rightarrow l\left(v_{\alpha}\right)$. Since $\left(s_{n}\right)$ is of flnite order, on application of Leme (3.53) shows that $s_{n} \rightarrow t_{\beta}\left(v_{\beta}\right), \beta=\frac{\alpha}{\alpha+1}$, and henice by Leme (3.s1), on $\rightarrow l\left(S_{1 /(a+1)}\right)$. As $0<\frac{1}{a+1}<1$, for any $\alpha>0$, we nay apply leme (4.1) once again to get the conclueion, $s_{n} \rightarrow \ell\left(C_{2 r}\right)$

(4.4) Corollary: If $a_{n}=s_{n}-s_{n-1}=o_{L}\left(n^{r-1 / 2}\right), r, 0$, and $\sum_{n=0}^{\infty} a_{n}=e(b, a, \beta), \operatorname{chén} \sum_{n=0}^{\infty} a_{n}=l\left(C_{2 r}\right)$.

Froof: By Reeark (2.5), $a_{n}=O_{L}\left(n^{r-1 / 2}\right.$, laplles $\left(T_{r}\right)$, and $\sum_{n=0}^{\infty} a_{n}=l(B, \alpha, \beta)$ is the sase as $\theta_{n} \rightarrow c(B, \alpha, \beta)$. So by Theorea (A.2). $s_{n} \rightarrow \ell\left(c_{2 r}\right)$, L.e. $\sum_{-n=0}^{\infty} a_{n}=e\left(c_{2 r}\right) \cdot 111$

Kuee ([15), Theorea 2) has given the corollary with O inatead of Q: $\rightarrow$

Recalling that the circle wethode consist of the $B, \dot{\nabla}_{B}, E_{a}, S_{\alpha}$. and $T_{a}$ eethods $(0<a<1, \beta>0$, , the O-version of Corollery (4.4), Theorea (3.21), and Corollaries (3.54), (3.55), (3.56) 1mply that : 
(4.5) Corollary: If $C$ is eny circle ecthod, $a_{n}=0\left(n^{r-1 / 2}\right), r, 0$, and $\sum_{n=0}^{\infty} i_{n}=$ e(c), then $\sum_{n=0}^{\infty} a_{n}=e\left(c_{2 r}\right)$.

$\cdot 3$

- Hote when C 18 the $B$ eethod or the $S_{a}$ nethod, the corollary 18 a apecial case of the results due to Ragagopal ([17], Theoren 2) and Sitaraman ([18], Theorea 2). 


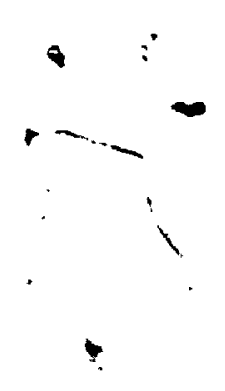

\section{-. prexigas}

[1]. Bacean Kanuacr ipt Project

"Higher Transcendental Fuactions", vol. 1. He Gram-H1L, (1953)

i2j Bingthen, H. H.: "Un Valiron and circle convergence:..

Xeth $2 ., 186,273-286 ;(1984)$

(3) Binghan. H. H. "Tauber 1on theorein tof amablitey methods of rendoe - wolk trpe".

Juurnal London hath. Soc., (2), 90, 28t-28is: (1944)

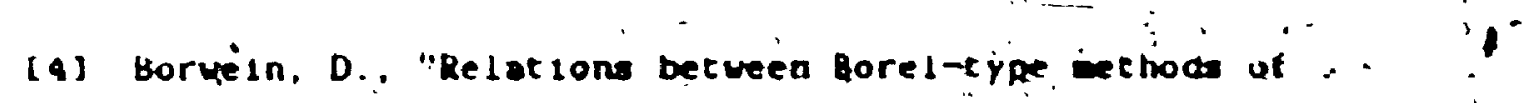
gumabllty".

Journa! London Math. Soc., 35, 65-70 H1500:

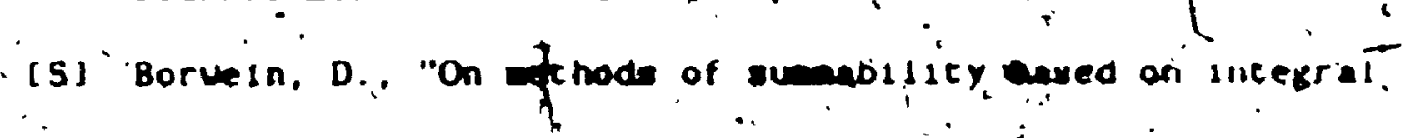
functions II".

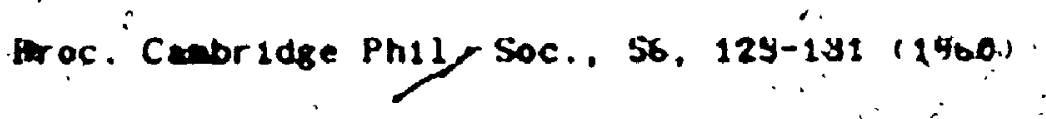

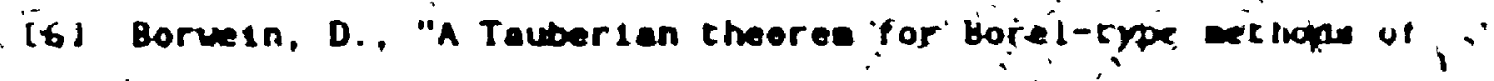
'oumat11ty.".

Cenadian Journal of path., 23, 740-747 (19\%9) -

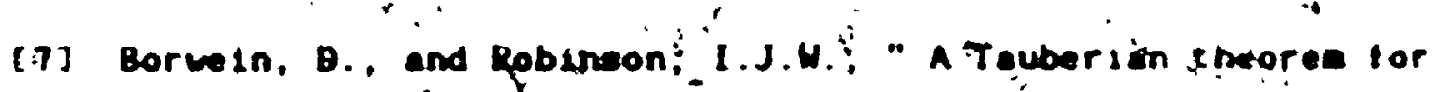
Borel-type ethode of eunability".

Journal Retne Areser. Wath. 273, 153-164 (i97s) 

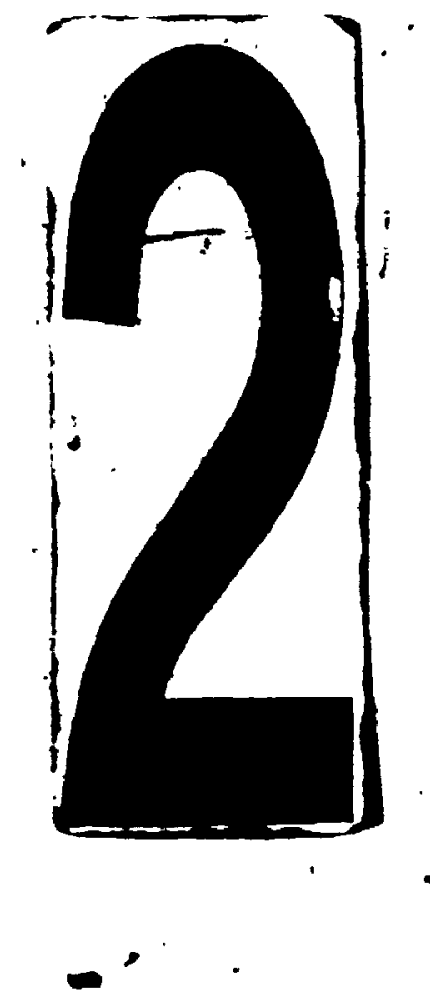

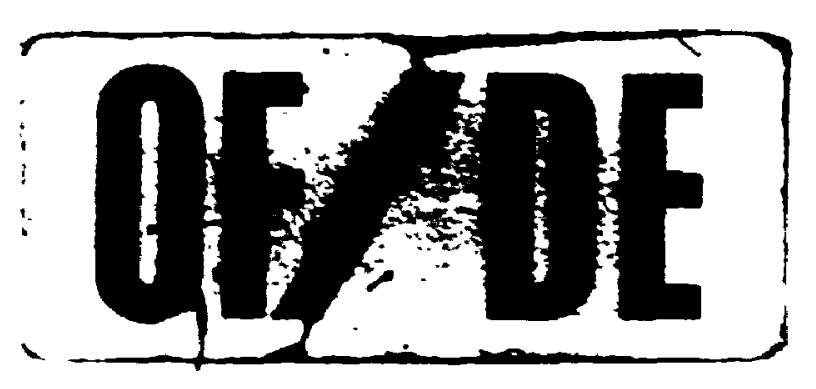

-

1
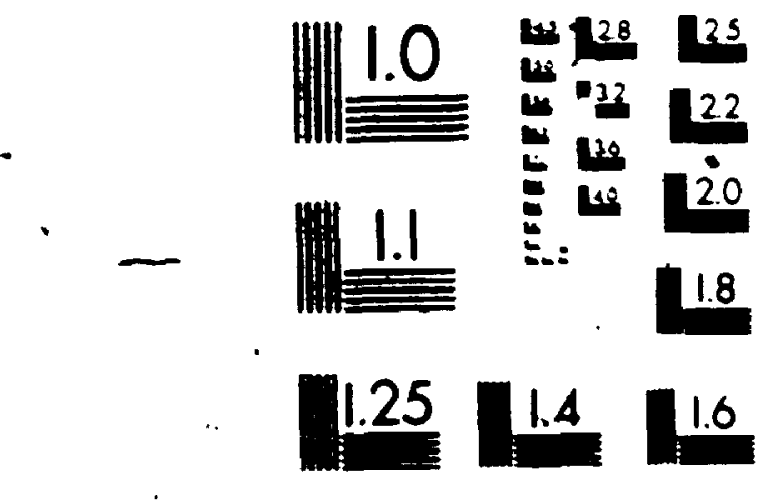

MICROCOPY RESOLUTION TEST CHART NATIONAL bUREAU OF STANDARDS (ANA

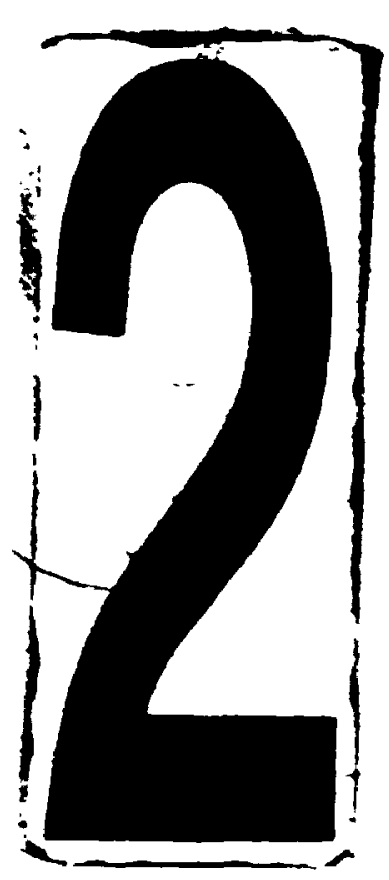

$-$ 
(8) Borwein, D., and Shawyer. B.L.R. "On Borei-type metroas".

Tohoku Math. Journal. 18, 283-298 (1966)

(9) Borvein. D., and Shatyer, B.L.R., "On Borel-type cetnous, II"

Tohoku Math. Journal, 19, 232-237 (1967)

(10) Faultiaber, G., "Aquivalenzsatze fur die kreisvertanten aer Liditerungstheorie".

- Matn. Z., 66, 34-52 (1956)

(11) Felier. W. "An Introduction to Probability Theory and 1ts Applications", vol.1, John wiley and Sons, Inc., (1950)

(12] Hardy, G.H., "Divergent Series".

Oxford (1949)

[13] H.rdy, G.H., and Littlewood, J.E. "Theorems concerning the sumabllity of sertes by Bopel's exponential sethod".

Rendicont 1 del Circolo Matematico di Palermo 41, 3b-sj (1916)

[14] Hyslop. J.M.,"The generaligation of a theored on Borel sumab1 $11 t y^{\prime \prime}$.

Proc. London Math. Soc. (2), 41, 243-256 (1936)

[15] Kuee, B.. "An Improvement on a theores of Hardy and Littlewood". Journal London Math. Soc. (2), 28, 93-102 (1983)

(16) Meyer-Konig, W., "Unterguchungen uber einige verwandte Lialt lerungsverfahren".

Math. Z., S2, 2S7-304 (1949)

[17] Kajagopal, C. T., "On a tneorea connecting Borel and Ceaaro sumabilit 1es". Journal. Indlan thath. Soc. 24, 433-442 (1960). 
(18) Sitaraman, $Y$. "On Tauberian theoreas for the $S_{a}$-thod of sumbilizir.

Hath. Z, 9S, 34-49 (1967)

(19) Vijayaraghavan. T., "A theorea concerning the sumability of a sertes by Borel'a wethod".

Proc. London Math. Soc. (2), 27, 316-326 (1928) 